\title{
Parameter identification and the multi-switching sliding mode combination synchronization of fractional order non-identical chaotic system under stochastic disturbances
}

\author{
Weiqiu Pan, Tianzeng Li; Yu Wang \\ College of Mathematics and Statistics, Sichuan University of Science and Engineering, \\ Zigong 643000, China
}

South Sichuan Center for Applied Mathematics, Yibin 644000, China.

\begin{abstract}
This paper deals with the issue of the multi-switching sliding mode combination synchronization (MSSMCS) of fractional order (FO) chaotic systems with different structures and unknown parameters under double stochastic disturbances (SD) utilizing the multi-switching synchronization method. The stochastic disturbances are considered as nonlinear uncertainties and external disturbances. Our theoretical part is divided into two cases, namely, the dimension of the drive-response system are different (or same). Firstly, a FO sliding surface was established in term of fractional calculus. Secondly, depended on the FO Lyapunov stability theory, the adaptive control technology and sliding mode control technique, the multi-switching adaptive controllers (MSAC) and some suitable multi-switching adaptive updating laws (MSAUL) are designed, so that the state variables of the drive systems are synchronized with the different state variables of the response systems. Simultaneously, the unknown parameters are assessed and the upper bound of stochastic disturbances are examined. Selecting the suitable scale matrices, the multi-switching projection synchronization, multi-switching complete synchronization, and multi-switching anti-synchronization will become special cases of MSSMCS. Finally, examples are displayed to certify the usefulness and validity of the demonstrated scheme via MATLAB.
\end{abstract}

Key words. Fractional-order chaotic system; Multi-switching combination synchronization (MSCS); Adaptive sliding mode control; Stochastic disturbance; Unknown parameters

\section{Introduction}

Chaos is an inherent characteristic of nonlinear dynamic systems and a common phenomenon in real life. The chaotic phenomenon exhibited by the chaotic system is

${ }^{*}$ Corresponding author. E-mail addresses: litianzeng27@163.com (T.Li), pwq237609@163.com (W.Pan), wangyu_813@163.com(Y.Wang) 
uncertain, unrepeatable, and unpredictable. Therefore, experts in the fields of mathematics and control have carried out a series of researches on the control and synchronization of chaotic systems. So far, some effective synchronization control methods have been proposed, such as drive-response synchronization [1], projection synchronization [2,3], adaptive fuzzy control [4-6], neural network synchronization [7,8], feedback synchronization [9], pulse synchronization [10,11], sliding mode control [12, 13] etc. People apply chaotic synchronization to secure communication, signal processing and life sciences, etc which has attracted great attention. Thus, chaos synchronization has gradually become a core research topic in the field of control science. Because chaotic systems are extremely sensitive to initial values, they are often subject to some SD. Whether it is the uncertainties within the system, such as parameter uncertainties, nonlinear uncertainties, or external disturbances, they cause an effect on the stability of the system. In the beginning, these synchronization methods are used by people to research the synchronization of single-drive system and single-response system [14 19. Gradually, some scholars have considered the influence of SD on this basis [20 27]. Since Runzi et al [28] revealed the combination synchronization scheme, the synchronization of multi-drive and multi-response systems, multi-drive and single-response systems, single-drive and multi-response systems are suggested. Later, some new synchronization schemes appeared and have been developed by leaps and bounds, such as the combination-combination [29 32, compound synchronization [33 35] and double compound synchronization [36, 37], etc. It can be said to be a major breakthrough in the field of chaos synchronization. A major advantage of these new synchronization schemes is that they can ensure the security of information. However, as the transmission of signals become more and more complex, how to strengthen the security of information is a thought-provoking question.

In recent years, a new multi-switching combination synchronization (MSCS) scheme was analyzed by Vincent $U$ et al [38] which means the state variables of the drive systems are synchronized with the different state variables of the response systems, breaking the conventional synchronization rules. Compared with the traditional synchronization or some extension of it, the MSCS scheme is very promising because it can provide greater security for the secure communication according to chaotic synchronization. The topic of dual combination-combination multi-switching synchronization in terms of eight chaotic systems was solved in 39. The global exponential MSCS was introduced in terms of three different chaotic systems [40]. Reference [41] solved the problem of MSCS between three different integer-order chaotic systems. Adopting adaptive control technology, reference [42] investigated the multi-switching combination-combination synchronization of four integer-order chaotic systems which parameters are fully unknown. An further work of [42] has been developed by [43], which is indicated as integer-order time-delay chaotic system. Shafiq M et al [44 proposed a robust adaptive multi-switching technology to solve the issue of anti-synchronization for unknown hyper-chaotic systems under SD. The authors of references [45] and [46] considered the multi-switching synchronization of the single-drive and single-response system which the parameters are unknown. Their innovations lie in the order of the drive-response system is different in [45] and the dimension is different in [46]. On the contrary, Chen et al [47] considered the synchronization of multiple chaotic systems with unknown parameters and disturbances. It's a pity that the case of multi-switching is not considered. Although reference [48] take multi-switching scheme into account in terms of 
multiple chaotic systems, it does not consider the influence of unknown parameters and SD.

Hence, to address this limitation, we plan to solve the multi-switching sliding mode combination synchronization (MSSMCS) of fractional order (FO) chaotic systems with different (or same) dimension under double stochastic disturbances (SD). Meanwhile, the parameters of two drive systems and one response system are fully unknown. The double SD are considered as nonlinear uncertainties and external disturbance. In the light of Lyapunov stability theory, adaptive control technology and sliding mode control technique, we introduce two multi-switching adaptive controllers (MSAC) and multi-switching adaptive updating laws (MSAUL) to realise the multiswitching synchronization of D-R systems and assess the unknown parameters. There are two innovations points in this article. 1. Considering the combination D-R systems with unknown parameters and double SD, the designed MSAC can make the state variables of drive systems are synchronized with the different state variables of the response system. Simultaneously, the designed MSAUL can identify the unknown parameters accurately and bounded estimates are made for uncertainties and external disturbances. 2. When the dimension of D-R systems are different (or same), scale matrices are chose as non-diagonal (or diagonal) matrices. If we adopt suitable scale matrices, the multiswitching projection synchronization, multi-switching complete synchronization, and multi-switching anti-synchronization will become the special cases of MSSMCS. Finally, numerical simulations via MATLAB demonstrate the multi-switching controllers we conducted have good robustness and anti-interference performance and this method we investigated can make the multi-switching error systems quickly converges to the equilibrium point. The rest of the paper is described as follows. In Section 2, some definitions, lemmas that need to be used are introduced. In Section 3, the problem statement are given. In Section 4, the MSAC and MSAUL are designed about D-R systems with the same dimension. In Section 5, the MSAC and MSAUL are designed about D-R systems with the different dimension. In Section 6, the numerical simulations conducted that our method is effective and dependable. In Section 7, there is a conclusion.

\section{Preliminaries}

The fractional calculus is an ancient and "fresh" concept. As early as the establishment of integer calculus, some scholars began to consider its meaning. Up till the present moment, there are some commonly used types of fractional derivatives, such as the Riemann-Liouville (R-L), Caputo and Grunwald-Letnikov (G-L) derivative etc. Among these definitions, Caputo's derivative definition is the most generally utilized.

Definition 1. 49 The mathematical expression of the fractional integral of the function $f(t)$ is following

$$
I_{t}^{\alpha} f(t)=\frac{1}{\Gamma(\alpha)} \int_{a}^{t} \frac{f(v)}{(t-v)^{1-\alpha}} d v,
$$

where $\Gamma(\alpha)$ indicates the Gamma function. 
Definition 2. [49] The mathematical expression of Caputo derivative with order $\alpha$ is given as

$$
{ }_{a}^{C} D_{t}^{\alpha} f(t)=\frac{1}{\Gamma(p-\alpha)} \int_{a}^{t}(t-v)^{p-\alpha-1} f^{(p)}(v) d v
$$

where $p-1<\alpha<p, p \in \mathbb{Z}^{+}$.

Lemma 1. [18] When $\boldsymbol{x}(t) \in \mathbb{R}^{n}$ has continuous first derivative, then

$$
{ }_{a} D_{t}^{\alpha}\left(\frac{1}{2} \boldsymbol{x}^{T}(t) Q \boldsymbol{x}(t)\right) \leq \boldsymbol{x}^{T} Q_{a} D_{t}^{\alpha} \boldsymbol{x}(t),
$$

where $\alpha \in(0,1)$ and $Q \in R^{n} \times R^{n}$ indicates a positive definite matrix.

\section{Problem description}

The FO D-R systems with SD are demonstrated as

$$
\begin{aligned}
& { }_{0} D_{t}^{\boldsymbol{\alpha}} \boldsymbol{x}(t)=\boldsymbol{F}(\boldsymbol{x}(t)) \boldsymbol{\theta}+\boldsymbol{f}(\boldsymbol{x}(t))+\Delta \boldsymbol{f}(\boldsymbol{x}(t))+\boldsymbol{d}(\boldsymbol{x}, t), \\
& { }_{0} D_{t}^{\boldsymbol{\alpha}} \boldsymbol{y}(t)=\boldsymbol{G}(\boldsymbol{y}(t)) \boldsymbol{\beta}+\boldsymbol{g}(\boldsymbol{y}(t))+\Delta \boldsymbol{g}(\boldsymbol{y}(t))+\boldsymbol{D}(\boldsymbol{y}, t),
\end{aligned}
$$

where the systems (44), (5) are regarded as drive systems. $\boldsymbol{x}(t)=\left(x_{1}, x_{2}, \cdots, x_{m}\right)^{T}$ and $\boldsymbol{y}(t)=\left(y_{1}, y_{2}, \cdots, y_{m}\right)^{T}$ are the state variables. $\boldsymbol{\theta}=\left(\theta_{1}, \theta_{2}, \cdots, \theta_{m}\right)^{T}$ and $\boldsymbol{\beta}=$ $\left(\beta_{1}, \beta_{2}, \cdots, \beta_{m}\right)^{T}$ are the parameter vectors; $\boldsymbol{F}_{k}(\boldsymbol{x}(t)),(k=1,2, \cdots, n)$ is the $k$ th row of $n \times m$ matrix $\boldsymbol{F}(\boldsymbol{x}(t))$ and $\boldsymbol{G}_{j}(\boldsymbol{y}(t)),(j=1,2, \cdots, n)$ is the $j$ th row of $n \times m$ matrix $\boldsymbol{G}(\boldsymbol{y}(t))$ whose elements are continuous nonlinear functions; $\boldsymbol{f}(\boldsymbol{x}(t))=$ $\left(f_{1}, f_{2}, \cdots, f_{m}\right)^{T}, \boldsymbol{g}(\boldsymbol{y}(t))=\left(g_{1}, g_{2}, \cdots, g_{m}\right)^{T}$ are the nonlinear continuous functions; $\boldsymbol{d}(\boldsymbol{x}(t))=\left(d_{1}, d_{2}, \cdots, d_{m}\right)^{T}, \boldsymbol{D}(\boldsymbol{y}(t))=\left(D_{1}, D_{2}, \cdots, D_{m}\right)^{T}$ are the external disturbances; $\Delta \boldsymbol{f}(\boldsymbol{x}(t))=\left(\Delta f_{1}, \Delta f_{2}, \cdots, \Delta f_{m}\right)^{T}, \Delta \boldsymbol{g}(\boldsymbol{y}(t))=\left(\Delta g_{1}, \Delta g_{2}, \cdots, \Delta g_{m}\right)^{T}$ are the nonlinear uncertainties; $\alpha_{i} \in(0,1)$ represents the fractional order.

$$
{ }_{0} D_{t}^{\boldsymbol{\alpha}} \boldsymbol{z}(t)=\boldsymbol{H}(\boldsymbol{z}(t)) \boldsymbol{\vartheta}+\boldsymbol{h}(\boldsymbol{z}(t))+\Delta \boldsymbol{h}(\boldsymbol{z}(t))+\boldsymbol{\mu}(\boldsymbol{z}, t)+\boldsymbol{u}(t),
$$

where the systems ([6) is regarded as response system. $\boldsymbol{z}(t)=\left(z_{1}, z_{2}, \cdots, z_{n}\right)^{T}$ and $\boldsymbol{\vartheta}=\left(\vartheta_{1}, \vartheta_{2}, \cdots, \vartheta_{n}\right)^{T}$ are the state variables and parameter vectors; $\boldsymbol{H}_{i}(\boldsymbol{z}(t)),(i=$ $1,2, \cdots, n)$ is the $i$ th row of $n \times n$ matrix $\boldsymbol{H}(\boldsymbol{z}(t))$ whose elements are continuous nonlinear functions; $\boldsymbol{h}(\boldsymbol{z}(t))=\left(h_{1}, h_{2}, \cdots, h_{n}\right)^{T}$ are the nonlinear continuous functions; $\boldsymbol{\mu}(\boldsymbol{z}(t))=\left(\mu_{1}, \mu_{2}, \cdots, \mu_{n}\right)^{T}$ are the external disturbances; $\Delta \boldsymbol{h}(\boldsymbol{z}(t))=$ $\left(\Delta h_{1}, \Delta h_{2}, \cdots, \Delta h_{n}\right)^{T}$ are the nonlinear uncertainties. $\boldsymbol{u}(t)=\left(u_{1}, u_{2}, \cdots, u_{n}\right)^{T}$ represent the controller.

Remark 1. It is worth noting that $\boldsymbol{x}(t), \boldsymbol{y}(t) \in \mathbb{R}^{m}, \boldsymbol{z}(t) \in \mathbb{R}^{n}, m=n$ or $m \neq n$; And the parameters of the D-R systems are unknown. We use $\hat{\boldsymbol{\theta}}, \hat{\boldsymbol{\beta}}, \hat{\boldsymbol{\vartheta}}$ to represent the estimation of parameter $\boldsymbol{\theta}, \boldsymbol{\beta}, \boldsymbol{\vartheta}$. 
Definition 3. If there exist three constant matrices $A \in \mathbb{R}^{n \times m}, B \in \mathbb{R}^{n \times m}, C \in$ $\mathbb{R}^{n \times n}, C \neq 0$ such that

$$
\lim _{t \rightarrow \infty}\|\boldsymbol{e}\|=\lim _{t \rightarrow \infty}\|C \boldsymbol{z}-(B \boldsymbol{y}+A \boldsymbol{x})\|=0,
$$

then the drive systems (4), (5) and response system (6) can be reach combination synchronization.

Remark 2. We can redefined the error system (7) as

$$
\lim _{t \rightarrow \infty}{ }^{(l w v)} e_{p}=\lim _{t \rightarrow \infty}\left[\sum_{i=1}^{n}\left(c_{l i} z_{i}\right)-\left\{\sum_{j=1}^{m}\left(b_{w j} y_{j}\right)+\sum_{k=1}^{m}\left(x_{v k} x_{k}\right)\right\}\right],
$$

where $i, j, k, p \in(1,2, \cdots, n)$ represent $p$ th error component of $\boldsymbol{e}, i$ th components of $\boldsymbol{z}, j$ th components of $\boldsymbol{y}$, and $k$ th components of $\boldsymbol{x}$ respectively, $l, w, v$ represent the switching mode. Suppose that $l=w=v$, then the error variables are expressed in the form of definition 3 ; if $l \neq w \neq v$, definition 3 will no longer apply.

Definition 4. We redefine the error state in definition 3 as

$$
\lim _{t \rightarrow \infty}{ }^{(l w v)} e_{p}=\lim _{t \rightarrow \infty}\left[\sum_{i=1}^{n}\left(c_{l i} z_{i}\right)-\left\{\sum_{j=1}^{m}\left(b_{w j} y_{j}\right)+\sum_{k=1}^{m}\left(x_{v k} x_{k}\right)\right\}\right]=0,
$$

then the drive systems (44), (5) and the response systems (6) realize the MSCS, where $l=w \neq v$ or $l \neq w=v$ or $l=w \neq v$ or $l \neq w \neq v$.

Remark 3. The matrices $A \in \mathbb{R}^{n \times m}, B \in \mathbb{R}^{n \times m}, C \in \mathbb{R}^{n \times n}, C \neq 0$ are hailed as the scaling matrices. Furthermore, they can be either constant matrices or the functions with regard to state variables $\boldsymbol{x}, \boldsymbol{y}, \boldsymbol{z}$.

Remark 4. If $A=B=C=I$, the MSCS becomes the multi-switching combination complete synchronization; If $A=0, B=C=I$ or $B=0, A=C=I$, the MSCS becomes the multi-switching complete synchronization.

Remark 5. If $A=B=-I, C=I$, the MSCS becomes the multi-switching combination anti-synchronization; If $A=0, B=-I, C=I$ or $B=0, A=-I, C=I$, the MSCS becomes the multi-switching anti-synchronization.

Remark 6. If $A \neq I, B \neq I, C=I$, the MSCS becomes the multi-switching combination projective synchronization; If $A=I, B \neq I, C=I$ or $B=I, A \neq I, C=I$, the MSCS becomes the multi-switching projective synchronization.

\section{The synchronization of multi-switching FO chaot- ic system with same dimensions}

In Section 4, the MSSMCS of FO chaotic systems with same dimension is formulated which means the dimension of D-R systems (44), (5) and (6) satisfies $m=n$. Thus, the scaling matrices $A, B, C$ are given as diagonal matrices. Firstly, we know 
that even if a chaotic system is slightly disturbed, its state orbit will change drastically over time. Therefore, it is crucial to suppose them as bounded. Then, we designed appropriate multi-switching adaptive controllers (MSAC) and some multi-switching adaptive updating laws (MSAUL) to realize the synchronization of the D-R system which are proved in Theorem 1.

When we choose $m=n$ and the diagonal matrices $A=\operatorname{diag}\left(a_{11}, a_{22}, \cdots, a_{n n}\right)$, $B=\operatorname{diag}\left(b_{11}, b_{22}, \cdots, b_{n n}\right), C=\operatorname{diag}\left(c_{11}, c_{22}, \cdots, c_{n n}\right)$, the error system (9) can be described as

$$
\lim _{t \rightarrow \infty}{ }^{(i j k)} e_{p}=\lim _{t \rightarrow \infty}\left[c_{i i} z_{i}-\left(b_{j j} y_{j}+a_{k k} x_{k}\right)\right]=0,
$$

where $i, j, k, p \in(1,2, \cdots, n)$ represent $p$ th error component of $\boldsymbol{e}, i$ th components of $\boldsymbol{z}, j$ th components of $\boldsymbol{y}$, and $k$ th components of $\boldsymbol{x}$.

Assumption 1. Assumed that the external disturbances $d_{k}, D_{j}, \mu_{i}$, uncertain nonlinear vectors $\Delta f_{k}, \Delta g_{j}, \Delta h_{i}$ all have a bounded norm. Namely, there are suitable positive constants ${ }^{(i j k)} r_{p},{ }^{(i j k)} q_{p}$ that satisfy

$$
\begin{aligned}
\left|c_{i i} \mu_{i}-\left(b_{j j} D_{j}+a_{k k} d_{k}\right)\right| & \leq{ }^{(i j k)} r_{p}, \\
\left|c_{i i} \Delta f_{k}-\left(b_{j j} \Delta g_{j}+a_{k k} \Delta h_{i}\right)\right| & \leq{ }^{(i j k)} q_{p} .
\end{aligned}
$$

where $p=1,2, \cdots, n$.

Remark 7. The positive constants ${ }^{(i j k)} r_{p},{ }^{(i j k)} q_{p}$ are unknown. ${ }^{(i j k)} \hat{r}_{p},{ }^{(i j k)} \hat{q}_{p}$ are used to represent the estimation of parameter ${ }^{(i j k)} r_{p}{ }^{\left({ }^{(i j k)}\right.} q_{p}$.

According to the definition of the error vector (10), we get the FO error system as

$$
\begin{aligned}
{ }_{0} D_{t}^{\alpha}\left\{{ }^{(i j k)} e_{p}\right\} & =c_{i i}\left\{{ }_{0} D_{t}^{\alpha} z_{i}\right\}-b_{j j}\left\{{ }_{0} D_{t}^{\alpha} y_{j}\right\}-a_{k k}\left\{{ }_{0} D_{t}^{\alpha} x_{k}\right\} \\
& =c_{i i} \boldsymbol{H}_{i}(z(t)) \boldsymbol{\vartheta}+c_{i i} h_{i}(z(t))+c_{i i} \Delta h_{i}(z(t))+c_{i i} \mu_{i}(z, t)+c_{i i}\left\{{ }^{(i j k)} u_{p}(t)\right\} \\
& -b_{j j} \boldsymbol{G}_{j}(y(t)) \boldsymbol{\beta}-b_{j j} g_{j}(y(t))-b_{j j} \Delta g_{j}(y(t))-b_{j j} D_{j}(y, t) \\
& -a_{k k} \boldsymbol{F}_{k}(x(t)) \boldsymbol{\theta}-a_{k k} f_{k}(x(t))-a_{k k} \Delta f_{k}(x(t))-a_{k k} d_{k}(x, t) .
\end{aligned}
$$

For convenience, we define the errors of unknown parameters $\boldsymbol{\theta}, \boldsymbol{\beta}, \boldsymbol{\vartheta},{ }^{(i j k)} r_{p},{ }^{(i j k)} q_{p}$ as $\tilde{\boldsymbol{\theta}}=\hat{\boldsymbol{\theta}}-\boldsymbol{\theta}, \tilde{\boldsymbol{\beta}}=\hat{\boldsymbol{\beta}}-\boldsymbol{\beta}, \tilde{\boldsymbol{\vartheta}}=\hat{\boldsymbol{\vartheta}}-\boldsymbol{\vartheta},{ }^{(i j k)} \tilde{r}_{p}={ }^{(i j k)} \hat{r}_{p}-{ }^{(i j k)} r_{p},{ }^{(i j k)} \tilde{q}_{p}={ }^{(i j k)} \hat{q}_{p}-{ }^{(i j k)} q_{p}$. When the sliding mode surface is designed as $\left.{ }^{(i j k)} s_{p}={ }^{(i j k)} \lambda_{p}{ }^{\left({ }^{(i j k)}\right.} e_{p}\right)$ which can be rewritten as $s=\left({ }^{(i j k)} s_{1},{ }^{(i j k)} s_{2}, \cdots,{ }^{(i j k)} s_{n}\right)$, we can get the following MSAC (13) and MSAUL (16) are designed:

$$
\begin{aligned}
{ }^{(i j k)} u_{p} & =\frac{1}{c_{i i}}\left\{-c_{i i} h_{i}+b_{j j} g_{j}+a_{k k} f_{k}+a_{k k} \boldsymbol{F}_{k}(x(t)) \hat{\boldsymbol{\theta}}+b_{j j} \boldsymbol{G}_{j}(y(t)) \hat{\boldsymbol{\beta}}\right. \\
& \left.-c_{i i} \boldsymbol{H}_{i}(z(t)) \hat{\boldsymbol{\vartheta}}-\left({ }^{(i j k)} r_{p}+{ }^{(i j k)} q_{p}\right) \operatorname{sign}\left({ }^{(i j k)} s_{p}\right)-k_{1} \tanh \left({ }^{(i j k)} s_{p}\right)\right\},
\end{aligned}
$$

where ${ }^{(i j k)} \lambda_{p}$ is a constant.

Substituting (13) into Eq. (12), we obtain

$$
\begin{aligned}
{ }_{0} D_{t}^{\alpha}\left\{{ }^{(i j k)} e_{p}\right\} & =-c_{i i} \boldsymbol{H}_{i}(z(t)) \tilde{\boldsymbol{\vartheta}}+b_{j j} \boldsymbol{G}_{j}(y(t)) \tilde{\boldsymbol{\beta}}+a_{k k} \boldsymbol{F}_{k}(x(t)) \tilde{\boldsymbol{\theta}} \\
& +\left\{c_{i i} \Delta h_{i}(z(t))-b_{j j} \Delta g_{j}(y(t))-x_{k k} \Delta f_{k}(x(t))\right\}+\left\{c_{i i} \mu_{i}(z, t)\right. \\
& \left.-b_{j j} D_{j}(y, t)-a_{k k} d_{k}(x, t)\right\}-\left({ }^{(i j k)} \hat{r}_{p}+{ }^{(i j k)} \hat{q}_{p}\right) \operatorname{sign}\left({ }^{(i j k)} s_{p}(t)\right) \\
& -k_{1} \tanh \left({ }^{(i j k)} s_{p}\right) .
\end{aligned}
$$


Thus, it follows from ${ }^{(i j k)} s_{p}={ }^{(i j k)} \lambda_{p}\left({ }^{(i j k)} e_{p}\right)$ and Eq. (14) that we obtain the following summation result.

$$
\begin{aligned}
& \sum_{p=1}^{n}\left\{{ }^{(i j k)} s_{p}\right\}_{0} D_{t}^{\alpha}\left\{{ }^{(i j k)} s_{p}\right\} \\
& =\sum_{i=1}^{n}{ }^{(i j k)} s_{i}\left({ }^{(i j k)} \lambda_{i}\right)\left\{-c_{i i} \boldsymbol{H}_{i}(z(t)) \tilde{\boldsymbol{\vartheta}}+c_{i i} \Delta h_{i}(z(t))+c_{i i} \mu_{i}(z, t)\right\} \\
& +\sum_{j=1}^{n}{ }^{(i j k)} s_{j}\left({ }^{(i j k)} \lambda_{j}\right)\left\{b_{j j} \boldsymbol{G}_{j}(y(t)) \tilde{\boldsymbol{\beta}}-b_{j j} \Delta g_{j}(y(t))-b_{j j} D_{j}(y, t)\right\} \\
& +\sum_{k=1}^{n}{ }^{(i j k)} s_{k}\left({ }^{(i j k)} \lambda_{p}\right)\left\{a_{k k} \boldsymbol{F}_{k}(x(t)) \tilde{\boldsymbol{\theta}}-a_{k k} \Delta f_{k}(x(t))-a_{k k} d_{k}(x, t)\right\} \\
& -\sum_{p=1}^{n}\left({ }^{(i j k)} \lambda_{p}\right)\left({ }^{(i j k)} \hat{r}_{p}+{ }^{(i j k)} \hat{q}_{p}\right) \operatorname{sign}\left({ }^{(i j k)} s_{p}(t)\right)-\sum_{p=1}^{n}\left({ }^{(i j k)} \lambda_{p}\right) k_{1} \tanh \left({ }^{(i j k)} s_{p}\right) .
\end{aligned}
$$

The MSAUL with regard to unknown parameters $\boldsymbol{\theta}, \boldsymbol{\beta}, \boldsymbol{\vartheta},{ }^{(i j k)} r_{p},{ }^{(i j k)} q_{p}$ are selected to

$$
\begin{aligned}
& { }_{0} D_{t}^{\alpha} \tilde{\boldsymbol{\theta}}=-\boldsymbol{F}^{T}(x(t)) \boldsymbol{\gamma}-\varphi_{1} \operatorname{sign}(\tilde{\boldsymbol{\theta}})|(\tilde{\boldsymbol{\theta}})|^{w}, \\
& { }_{0} D_{t}^{\alpha} \tilde{\boldsymbol{\beta}}=-\boldsymbol{G}^{T}(y(t)) \boldsymbol{\xi}-\varphi_{2} \operatorname{sign}(\tilde{\boldsymbol{\beta}})|(\tilde{\boldsymbol{\beta}})|^{w}, \\
& { }_{0} D_{t}^{\alpha} \tilde{\boldsymbol{\vartheta}}=\boldsymbol{H}^{T}(z(t)) \varpi-\varphi_{3} \operatorname{sign}(\tilde{\boldsymbol{\vartheta}})|(\tilde{\boldsymbol{\vartheta}})|^{w}, \\
& { }_{0} D_{t}^{\alpha}\left\{{ }^{(i j k)} \tilde{r}_{p}\right\}=\left.m_{1}\left({ }^{(i j k)} \lambda_{p}\right)\right|^{(i j k)} s_{p}(t) \mid, \\
& { }_{0} D_{t}^{\alpha}\left\{{ }^{(i j k)} \tilde{q}_{p}\right\}=\left.m_{2}\left({ }^{(i j k)} \lambda_{p}\right)\right|^{(i j k)} s_{p}(t) \mid,
\end{aligned}
$$

where

$$
\begin{aligned}
& \gamma=\left\{\left({ }^{(i j k)} \lambda_{1}\right) a_{11}^{*}\left({ }^{(i j k)} s_{1}\right),\left({ }^{(i j k)} \lambda_{2}\right) a_{22}^{*}\left({ }^{(i j k)} s_{2}\right), \cdots,\left({ }^{(i j k)} \lambda_{n}\right) a_{n n}^{*}\left({ }^{(i j k)} s_{n}\right)^{T}\right\} \\
& \boldsymbol{\xi}=\left\{\left({ }^{(i j k)} \lambda_{1}\right) b_{11}^{*}\left({ }^{(i j k)} s_{1}\right),\left({ }^{(i j k)} \lambda_{2}\right) b_{22}^{*}\left({ }^{(i j k)} s_{2}\right), \cdots,\left({ }^{(i j k)} \lambda_{n}\right) b_{n n}^{*}\left({ }^{(i j k)} s_{n}\right)^{T}\right\}, \\
& \varpi=\left\{\left({ }^{(i j k)} \lambda_{1}\right) c_{11}^{*}\left({ }^{(i j k)} s_{1}\right),\left({ }^{(i j k)} \lambda_{2}\right) c_{22}^{*}\left({ }^{(i j k)} s_{2}\right), \cdots,\left({ }^{(i j k)} \lambda_{n}\right) c_{n n}^{*}\left({ }^{(i j k)} s_{n}\right)^{T}\right\},
\end{aligned}
$$

$m_{1}, m_{2}$ are positive constants and the meaning of matrices $A^{*}=\operatorname{diag}\left(a_{11}^{*}, a_{22}^{*}, \cdots, a_{n n}^{*}\right)$, $B^{*}=\operatorname{diag}\left(b_{11}^{*}, b_{22}^{*}, \cdots, b_{n n}^{*}\right), C^{*}=\operatorname{diag}\left(c_{11}^{*}, c_{22}^{*}, \cdots, c_{n n}^{*}\right)$ is the rearrangement of the original matrices $A, B, C$.

Theorem 1. For any given initial conditions $\boldsymbol{x}(0), \boldsymbol{y}(0), \boldsymbol{z}(0)$, if the Assumption 1 is hold, the synchronization error system (10) will achieve the multi-switching sliding mode combination synchronization (MSSMCS) via the multi-switching adaptive controller (MSAC) (13) and multi-switching adaptive updating laws (MSAUL) (16).

Proof. Adopting the Lyapunov function as:

$$
\begin{aligned}
V & =\frac{1}{2} \sum_{p=1}^{n}{ }^{(i j k)} s_{p}^{2}+\frac{1}{2 m_{1}} \sum_{p=1}^{n}\left\{{ }^{(i j k)} \tilde{r}_{p}^{T}\right\}\left\{{ }^{(i j k)} \tilde{r}_{p}\right\}+\frac{1}{2 m_{2}} \sum_{p=1}^{n}\left\{{ }^{(i j k)} \tilde{q}_{p}^{T}\right\}\left\{{ }^{(i j k)} \tilde{q}_{p}\right\} \\
& +\frac{1}{2} \tilde{\boldsymbol{\theta}}^{T} \boldsymbol{\theta}+\frac{1}{2} \tilde{\boldsymbol{\beta}}^{T} \boldsymbol{\beta}+\frac{1}{2} \tilde{\boldsymbol{\vartheta}}^{T} \boldsymbol{\vartheta} .
\end{aligned}
$$


Taking the $\alpha$ derivative

$$
\begin{aligned}
{ }_{0} D_{t}^{\alpha} V(t, \boldsymbol{x}(t)) & \leq \sum_{p=1}^{n}\left\{{ }^{(i j k)} s_{p}\right\}_{0} D_{t}^{\alpha}\left\{{ }^{(i j k)} s_{p}\right\}+\frac{1}{m_{1}} \sum_{p=1}^{n}\left\{{ }^{(i j k)} \tilde{r}_{p}^{T}\right\}_{0} D_{t}^{\alpha}\left\{{ }^{(i j k)} \tilde{r}_{p}\right\} \\
& +\frac{1}{m_{2}} \sum_{p=1}^{n}\left\{{ }^{(i j k)} \tilde{q}_{p}^{T}\right\}_{0} D_{t}^{\alpha}\left\{{ }^{(i j k)} \tilde{q}_{p}\right\}+\tilde{\boldsymbol{\theta}}^{T}{ }_{0} D_{t}^{\alpha} \tilde{\boldsymbol{\theta}}+\tilde{\boldsymbol{\beta}}^{T}{ }_{0} D_{t}^{\alpha} \tilde{\boldsymbol{\beta}}+\tilde{\boldsymbol{\vartheta}}^{T}{ }_{0} D_{t}^{\alpha} \tilde{\boldsymbol{\vartheta}}
\end{aligned}
$$

Then substituting the Eq. (15) and the MSAUL (16) into Eq. (18), we obtain

$$
\begin{aligned}
{ }_{0} D_{t}^{\alpha} V(t, \boldsymbol{x}(t)) \leq & \sum_{i=1}^{n}{ }^{(i j k)} s_{i}\left({ }^{(i j k)} \lambda_{i}\right)\left(-c_{i i} \boldsymbol{H}_{i}(z(t)) \tilde{\boldsymbol{\vartheta}}+c_{i i} \Delta h_{i}(z(t))+c_{i i} \mu_{i}(z, t)\right) \\
& +\sum_{j=1}^{n}{ }^{(i j k)} s_{j}\left({ }^{(i j k)} \lambda_{j}\right)\left(b_{j j} \boldsymbol{G}_{j}(y(t)) \tilde{\boldsymbol{\beta}}-b_{j j} \Delta g_{j}(y(t))-b_{j j} D_{j}(y, t)\right) \\
& +\sum_{k=1}^{n}{ }^{(i j k)} s_{k}\left({ }^{\left({ }^{(i j k)}\right.} \lambda_{k}\right)\left(a_{k k} \boldsymbol{F}_{k}(x(t)) \tilde{\boldsymbol{\theta}}-a_{k k} \Delta f_{k}(x(t))-a_{k k} d_{k}(x, t)\right) \\
& -\sum_{p=1}^{n}\left({ }^{(i j k)} \lambda_{p}\right)\left({ }^{(i j k)} \hat{r}_{p}+{ }^{(i j k)} \hat{q}_{p}\right) \operatorname{sign}\left({ }^{(i j k)} s_{p}(t)\right)-\sum_{p=1}^{n}\left({ }^{(i j k)} \lambda_{p}\right) k_{1} \tanh \left({ }^{(i j k)} s_{p}\right) \\
& +\tilde{\boldsymbol{\theta}}^{T}\left(-\boldsymbol{F}^{T}(x(t)) \boldsymbol{\gamma}-\varphi_{1} \operatorname{sign}(\tilde{\boldsymbol{\theta}})|(\tilde{\boldsymbol{\theta}})|^{w}\right) \\
& +\tilde{\boldsymbol{\beta}}^{T}\left(-\boldsymbol{G}^{T}(y(t)) \boldsymbol{\xi}-\varphi_{2} \operatorname{sign}(\tilde{\boldsymbol{\beta}})|(\tilde{\boldsymbol{\beta}})|^{w}\right) \\
& +\tilde{\boldsymbol{\vartheta}}^{T}\left(\boldsymbol{H}^{T}(z(t)) \varpi-\varphi_{3} \operatorname{sign}(\tilde{\boldsymbol{\vartheta}})|(\tilde{\boldsymbol{\vartheta}})|^{w}\right) \\
& +\sum_{p=1}^{n}{ }^{(i j k)} \tilde{r}_{p}^{T}\left({ }^{(i j k)} \lambda_{p}\right)\left(\left|{ }^{(i j k)} s_{p}\right|\right)+\sum_{p=1}^{\left({ }^{(i j k)}\right.} \tilde{q}_{p}^{T}\left({ }^{(i j k)} \lambda_{p}\right)\left(\left.\right|^{(i j k)} s_{p} \mid\right) .
\end{aligned}
$$

Since

$$
\begin{aligned}
& \sum_{j=1}^{n}{ }^{(i j k)} s_{j}\left({ }^{(i j k)} \lambda_{j}\right) b_{j j} \boldsymbol{G}_{j}(y(t)) \tilde{\boldsymbol{\beta}}=\tilde{\boldsymbol{\beta}}^{T} \boldsymbol{G}^{T}(y(t)) \boldsymbol{\xi} \\
& \sum_{k=1}^{n}{ }^{\left({ }^{(i j k)}\right.} s_{k}\left({ }^{(i j k)} \lambda_{k}\right) a_{k k} \boldsymbol{F}_{k}(x(t)) \tilde{\boldsymbol{\theta}}=\tilde{\boldsymbol{\theta}}^{T} \boldsymbol{F}^{T}(x(t)) \boldsymbol{\gamma} \\
& \sum_{i=1}^{n}{ }^{(i j k)} s_{i}\left({ }^{(i j k)} \lambda_{i}\right) c_{i i} \boldsymbol{H}_{i}(z(t)) \tilde{\boldsymbol{\vartheta}}=\tilde{\boldsymbol{\vartheta}}^{T} \boldsymbol{H}^{T}(z(t)) \varpi .
\end{aligned}
$$

Thus

$$
\begin{aligned}
{ }_{0} D_{t}^{\alpha} V(t, \boldsymbol{x}(t)) & \leq \sum_{i=1}^{n}{ }^{(i j k)} s_{i}\left({ }^{(i j k)} \lambda_{i}\right)\left(c_{i i} \Delta h_{i}(z(t))+c_{i i} \mu_{i}(z, t)\right) \\
& +\sum_{j=1}^{n}{ }^{(i j k)} s_{j}\left({ }^{(i j k)} \lambda_{j}\right)\left(-b_{j j} \Delta g_{j}(y(t))-b_{j j} D_{j}(y, t)\right) \\
& +\sum_{k=1}^{n}{ }^{(i j k)} s_{k}\left({ }^{(i j k)} \lambda_{k}\right)\left(-a_{k k} \Delta f_{k}(x(t))-a_{k k} d_{k}(x, t)\right)
\end{aligned}
$$




$$
\begin{aligned}
& -\tilde{\boldsymbol{\theta}}^{T}\left(\varphi_{1} \operatorname{sign}(\tilde{\boldsymbol{\theta}})|(\tilde{\boldsymbol{\theta}})|^{w}\right)-\tilde{\boldsymbol{\beta}}^{T}\left(\varphi_{2} \operatorname{sign}(\tilde{\boldsymbol{\beta}})|(\tilde{\boldsymbol{\beta}})|^{w}\right)-\tilde{\boldsymbol{\vartheta}}^{T}\left(\varphi_{3} \operatorname{sign}(\tilde{\boldsymbol{\vartheta}})|(\tilde{\boldsymbol{\vartheta}})|^{w}\right) \\
& -\sum_{p=1}^{n}\left({ }^{(i j k)} \lambda_{p}\right)\left({ }^{(i j k)} \hat{r}_{p}+{ }^{(i j k)} \hat{q}_{p}\right) \operatorname{sign}\left({ }^{(i j k)} s_{p}(t)\right)-\sum_{p=1}^{n}\left({ }^{(i j k)} \lambda_{p}\right) k_{1} \tanh \left({ }^{(i j k)} s_{p}\right) \\
& +\sum_{p=1}^{n}\left({ }^{(i j k)} \lambda_{p}\right)\left({ }^{(i j k)} \tilde{r}_{p}^{T}\right)\left(\left|{ }^{(i j k)} s_{p}\right|\right)+\sum_{p=1}^{n}\left({ }^{(i j k)} \lambda_{p}\right)\left({ }^{(i j k)} \tilde{q}_{p}^{T}\right)\left(\left|{ }^{(i j k)} s_{p}\right|\right) \\
& \leq\left.\sum_{p=1}^{n}\left({ }^{(i j k)} \lambda_{p}\right)\left({ }^{(i j k)} r_{p}\right)\right|^{(i j k)} s_{p}\left|+\sum_{p=1}^{n}\left({ }^{(i j k)} \lambda_{p}\right)\left({ }^{(i j k)} q_{p}\right)\right|^{(i j k)} s_{p} \mid \\
& -\left.\sum_{p=1}^{n}\left({ }^{(i j k)} \lambda_{p}\right)\left({ }^{(i j k)} \hat{r}_{p}+{ }^{(i j k)} \hat{q}_{p}\right)\right|^{(i j k)} s_{p} \mid+\sum_{p=1}^{n}\left({ }^{(i j k)} \lambda_{p}\right)\left({ }^{(i j k)} \tilde{r}_{p}^{T}\right)\left(\left|{ }^{(i j k)} s_{p}\right|\right) \\
& +\sum_{p=1}^{n}\left({ }^{(i j k)} \lambda_{p}\right)\left({ }^{(i j k)} \tilde{q}_{p}^{T}\right)\left({ }^{(i j k)} s_{p} \mid\right)-\tilde{\boldsymbol{\theta}}^{T}\left(\varphi_{1} \operatorname{sign}(\tilde{\boldsymbol{\theta}})|(\tilde{\boldsymbol{\theta}})|^{w}\right) \\
& -\tilde{\boldsymbol{\beta}}^{T}\left(\varphi_{2} \operatorname{sign}(\tilde{\boldsymbol{\beta}})|(\tilde{\boldsymbol{\beta}})|^{w}\right)-\tilde{\boldsymbol{\vartheta}}^{T}\left(\varphi_{3} \operatorname{sign}(\tilde{\boldsymbol{\vartheta}})|(\tilde{\boldsymbol{\vartheta}})|^{w}\right) \\
& -\sum_{i=1}^{n}{ }^{(i j k)} s_{p}\left({ }^{(i j k)} \lambda_{p}\right) k_{1} \tanh \left({ }^{(i j k)} s_{p}\right) \\
& =-\tilde{\boldsymbol{\theta}}^{T}\left(\varphi_{1} \operatorname{sign}(\tilde{\boldsymbol{\theta}})|(\tilde{\boldsymbol{\theta}})|^{w}\right)-\tilde{\boldsymbol{\beta}}^{T}\left(\varphi_{2} \operatorname{sign}(\tilde{\boldsymbol{\beta}})|(\tilde{\boldsymbol{\beta}})|^{w}\right) \\
& -\tilde{\boldsymbol{\vartheta}}^{T}\left(\varphi_{3} \operatorname{sign}(\tilde{\boldsymbol{\vartheta}})|(\tilde{\boldsymbol{\vartheta}})|^{w}\right)-\sum_{i=1}^{p}{ }^{(i j k)} s_{p} k_{1} \tanh \left(\lambda^{(i j k)} s_{p}\right) \text {. }
\end{aligned}
$$

Which establishes that the ${ }_{0} D_{t}^{\alpha} V(t, \boldsymbol{x}(t))$ is negative definite. Thus, it follows from the FO Lyapunov stability theory that $\lim _{t \rightarrow \infty}{ }^{(i j k)} e_{p}=0$ is achieved, i.e. we can say that the MSSMCS of the drive systems (4), (5) and response system (6) is accomplished in terms of $m=n$.

\section{The synchronization of multi-switching FO sys- tem with different dimensions}

In Section 5, the MSSMCS of FO chaotic systems with different dimension is formulated which means the dimension of D-R systems (4), (5) and (6) satisfies $m \neq n$. Thus, the scaling matrices $A, B, C$ are given as non-diagonal matrices. We designed appropriate multi-switching adaptive controllers (MSAC) and some multi-switching adaptive updating laws (MSAUL) to realize the synchronization of the D-R system which are proved in Theorem 2.

When we choose $m \neq n$ and the non-diagonal matrices $A \in \mathbb{R}^{n \times m}, B \in \mathbb{R}^{n \times m}, C \in$ $\mathbb{R}^{n \times n}, C \neq 0$, the form of the error system (9) can be explained as:

$$
\lim _{t \rightarrow \infty}{ }^{(l w v)} e_{p}=\lim _{t \rightarrow \infty}\left[\sum_{i=1}^{n}\left(c_{l i} z_{i}\right)-\left\{\sum_{j=1}^{m}\left(b_{w j} y_{j}\right)+\sum_{k=1}^{m}\left(x_{v k} x_{k}\right)\right\}\right]=0 .
$$

Assumption 2. Assumed that the external disturbances $d_{k}, D_{j}, \mu_{i}$, uncertain nonlinear vectors $\Delta f_{k}, \Delta g_{j}, \Delta h_{i}$ all have a bounded norm. Namely, there are suitable 
positive constants ${ }^{(l w v)} \rho_{p},{ }^{(l w v)} \varrho_{p}$ that satisfy

$$
\begin{array}{r}
\left|\sum_{i=1}^{n} c_{l i} \mu_{i}-\left(\sum_{j=1}^{m} b_{w j} D_{j}+\sum_{k=1}^{m} x_{v k} d_{k}\right)\right| \leq{ }^{(l w v)} \rho_{p}, \\
\left|\sum_{i=1}^{n} c_{l i} \Delta h_{i}-\left(\sum_{j=1}^{m} b_{w j} \Delta g_{j}+\sum_{k=1}^{m} x_{v k} \Delta f_{k}\right)\right| \leq{ }^{(l w v)} \varrho_{p},
\end{array}
$$

where $p=1,2, \cdots, n$.

Remark 8. The positive constants ${ }^{(l w v)} \rho_{p},{ }^{(l w v)} \varrho_{p}$ are unknown, ${ }^{(l w v)} \hat{\rho}_{p},{ }^{(l w v)} \hat{\varrho}_{p}$ are used to represent the estimation of parameter ${ }^{(l w v)} \rho_{p},{ }^{(l w v)} \varrho_{p}$.

According to the definition of the error vector (22), we get the FO error system as

$$
\begin{aligned}
{ }_{0} D_{t}^{\alpha}\left\{{ }^{(l w v)} e_{p}\right\} & =\sum_{i=1}^{n} c_{l i}\left\{{ }_{0} D_{t}^{\alpha} z_{i}\right\}-\sum_{j=1}^{m} b_{w j}\left\{{ }_{0} D_{t}^{\alpha} y_{j}\right\}-\sum_{k=1}^{m} x_{v k}\left\{{ }_{0} D_{t}^{\alpha} x_{k}\right\} \\
& =\sum_{i=1}^{n} c_{l i}\left\{\boldsymbol{H}_{i}(z(t)) \boldsymbol{\vartheta}+h_{i}(z(t))+\Delta h_{i}(z(t))+\mu_{i}(z, t)+{ }^{(l w v)} u_{p}(t)\right\} \\
& -\sum_{j=1}^{m} b_{w j}\left\{\boldsymbol{G}_{j}(y(t)) \boldsymbol{\beta}+g_{j}(y(t))+\Delta g_{j}(y(t))+D_{j}(y, t)\right\} \\
& -\sum_{k=1}^{m} x_{v k}\left\{\boldsymbol{F}_{k}(x(t)) \boldsymbol{\theta}+f_{k}(x(t))+\Delta f_{k}(x(t))+d_{k}(x, t)\right\} .
\end{aligned}
$$

The errors of unknown parameters $\boldsymbol{\theta}, \boldsymbol{\beta}, \boldsymbol{\vartheta}$ have been defined in (12). For convenience, we define error of unknown constants ${ }^{(l w v)} \rho_{p},{ }^{(l w v)} \varrho_{p}$ as ${ }^{(l w v)} \tilde{\rho}_{p}={ }^{(l w v)} \hat{\rho}_{p}-{ }^{(l w v)} \rho_{p}$, ${ }^{(l w v)} \tilde{\varrho}_{p}={ }^{(l w v)} \hat{\varrho}_{p}-{ }^{(l w v)} \varrho_{p}$. Thus, the sliding mode surface is designed as ${ }^{(l w v)} s_{p}={ }^{(l w v)}$ $\lambda_{p}\left({ }^{(l w v)} e_{p}\right)$. We can get the following multi-switching adaptive controller (MSAC) (25) multi-switching adaptive updating laws (MSAUL) (28):

$$
\begin{aligned}
\left.\sum_{i=1}^{n} c_{l i}{ }^{(l w v)} u_{p}\right\} & =-\sum_{i=1}^{n} c_{l i} h_{i}+\sum_{j=1}^{m} b_{w j} g_{j}+\sum_{k=1}^{m} x_{v k} f_{k}+\sum_{k=1}^{m} x_{v k} \boldsymbol{F}_{k}(x(t)) \hat{\boldsymbol{\theta}} \\
& +\sum_{j=1}^{m} b_{w j} \boldsymbol{G}_{j}(y(t)) \hat{\boldsymbol{\beta}}-\sum_{i=1}^{n} c_{l i} \boldsymbol{H}_{i}(z(t)) \hat{\boldsymbol{\vartheta}}-\left({ }^{(l w v)} \hat{\rho}_{p}+{ }^{(l w v)} \hat{\varrho}_{p}\right) \operatorname{sign}\left({ }^{(l w v)} s_{p}\right) \\
& -k_{1} \tanh \left({ }^{(l w v)} s_{p}\right)
\end{aligned}
$$

where ${ }^{(l w v)} \lambda_{p}$ is a constant. Substituting (25) into Eq. (24), we obtain

$$
\begin{aligned}
{ }_{0} D_{t}^{\alpha}\left\{{ }^{(l w v)} e_{p}\right\} & =\sum_{i=1}^{n} c_{l i}\left\{-\boldsymbol{H}_{i}(z(t)) \tilde{\boldsymbol{\vartheta}}+\Delta h_{i}(z(t))+\mu_{i}(z, t)\right\} \\
& +\sum_{j=1}^{m} b_{w j}\left\{\boldsymbol{G}_{j}(y(t)) \tilde{\boldsymbol{\beta}}+\Delta g_{j}(y(t))+D_{j}(y, t)\right\} \\
& +\sum_{k=1}^{m} x_{v k}\left\{\boldsymbol{F}_{k}(x(t)) \tilde{\boldsymbol{\theta}}+\Delta f_{k}(x(t))+d_{k}(x, t)\right\}
\end{aligned}
$$




$$
-\left({ }^{(l w v)} \hat{\rho}_{p}+{ }^{(l w v)} \hat{\varrho}_{p}\right) \operatorname{sign}\left({ }^{(l w v)} s_{p}\right)-k_{1} \tanh \left({ }^{(l w v)} s_{p}\right) .
$$

Thus, it follows from ${ }^{(l w v)} s_{p}={ }^{(l w v)} \lambda_{p}\left({ }^{(l w v)} e_{p}\right)$ and Eq. (26) that we obtain the following summation result.

$$
\begin{aligned}
& \sum_{p=1}^{n}\left\{{ }^{(l w v)} s_{p}\right\}_{0} D_{t}^{\alpha}\left\{{ }^{(l w v)} s_{p}\right\} \\
& =\sum_{l=1}^{n} \sum_{i=1}^{n}{ }^{(l w v)} s_{i}\left({ }^{(l w v)} \lambda_{i}\right) c_{l i}\left\{-\boldsymbol{H}_{i}(z(t)) \tilde{\boldsymbol{\vartheta}}+\Delta h_{i}(z(t))+\mu_{i}(z, t)\right\} \\
& +\sum_{w=1}^{n} \sum_{j=1}^{m}{ }^{(l w v)} s_{j}\left({ }^{(l w v)} \lambda_{j}\right) b_{w j}\left\{\boldsymbol{G}_{j}(y(t)) \tilde{\boldsymbol{\beta}}+\Delta g_{j}(y(t))+D_{j}(y, t)\right\} \\
& +\sum_{v=1}^{n} \sum_{k=1}^{m}{ }^{(l w v)} s_{k}\left({ }^{(l w v)} \lambda_{k}\right) x_{v k}\left\{\boldsymbol{F}_{k}(x(t)) \tilde{\boldsymbol{\theta}}+\Delta f_{k}(x(t))+d_{k}(x, t)\right\} \\
& -\sum_{p=1}^{n}\left({ }^{(l w v)} \lambda_{p}\right)\left({ }^{(l w v)} \hat{\rho}_{p}+{ }^{(l w v)} \hat{\varrho}_{p}\right) \operatorname{sign}\left({ }^{(l w v)} s_{p}(t)\right)-\sum_{p=1}^{n}\left({ }^{(l w v)} \lambda_{p}\right) k_{1} \tanh \left({ }^{(l w v)} s_{p}\right) .
\end{aligned}
$$

The MSAUL with regard to unknown parameters $\boldsymbol{\theta}, \boldsymbol{\beta}, \boldsymbol{\vartheta},{ }^{(l w v)} \rho_{p},{ }^{(l w v)} \varrho_{p}$ are selected as

$$
\begin{aligned}
& { }_{0} D_{t}^{\alpha} \tilde{\boldsymbol{\theta}}=-\boldsymbol{F}^{T}(x(t)) \boldsymbol{\gamma}-\varphi_{1} \operatorname{sign}(\tilde{\boldsymbol{\theta}})|(\tilde{\boldsymbol{\theta}})|^{w}, \\
& { }_{0} D_{t}^{\alpha} \tilde{\boldsymbol{\beta}}=-\boldsymbol{G}^{T}(y(t)) \boldsymbol{\xi}-\varphi_{2} \operatorname{sign}(\tilde{\boldsymbol{\beta}})|(\tilde{\boldsymbol{\beta}})|^{w}, \\
& { }_{0} D_{t}^{\alpha} \tilde{\boldsymbol{\vartheta}}=\boldsymbol{H}^{T}(z(t)) \varpi-\varphi_{3} \operatorname{sign}(\tilde{\boldsymbol{\vartheta}})|(\tilde{\boldsymbol{\vartheta}})|^{w}, \\
& { }_{0} D_{t}^{\alpha}\left\{{ }^{(l w v)} \tilde{\rho}_{p}\right\}=\left.m_{1}\left({ }^{(l w v)} \lambda_{p}\right)\right|^{(l w v)} s_{p}(t) \mid, \\
& { }_{0} D_{t}^{\alpha}\left\{{ }^{(l w v)} \tilde{\varrho}_{p}\right\}=\left.m_{2}\left({ }^{(l w v)} \lambda_{p}\right)\right|^{(l w v)} s_{p}(t) \mid,
\end{aligned}
$$

where

$$
\begin{gathered}
\boldsymbol{\gamma}=\left\{\sum_{k=1}^{m}\left({ }^{(l w v)} \lambda_{1}\right) x_{v k}\left({ }^{(l w v)} s_{1}\right), \sum_{k=1}^{m}\left({ }^{(l w v)} \lambda_{2}\right) x_{v k}\left({ }^{(l w v)} s_{2}\right), \cdots, \sum_{k=1}^{m}\left({ }^{(l w v)} \lambda_{n}\right) x_{v k}\left({ }^{(l w v)} s_{n}\right)\right\}^{T}, \\
\boldsymbol{\xi}=\left(\sum_{j=1}^{m}\left({ }^{(l w v)} \lambda_{1}\right) b_{w j}\left({ }^{(l w v)} s_{1}\right), \sum_{j=1}^{m}\left({ }^{(l w v)} \lambda_{2}\right) b_{w j}\left({ }^{(l w v)} s_{2}\right), \cdots, \sum_{j=1}^{m}\left({ }^{(l w v)} \lambda_{n}\right) b_{w j}\left({ }^{(l w v)} s_{n}\right)\right)^{T}, \\
\varpi=\left(\sum_{i=1}^{n}\left({ }^{(l w v)} \lambda_{1}\right) c_{l i}{ }^{(l w v)} s_{1}, \sum_{i=1}^{n}\left({ }^{(l w v)} \lambda_{2}\right) c_{l i}\left({ }^{(l w v)} s_{2}\right), \cdots, \sum_{i=1}^{n}\left({ }^{(l w v)} \lambda_{n}\right) c_{l i}\left({ }^{(l w v)} s_{n}\right)\right)^{T},
\end{gathered}
$$

$m_{1}, m_{2}$ are positive constants.

Theorem 2. For any given initial conditions $\boldsymbol{x}(0), \boldsymbol{y}(0), \boldsymbol{z}(0)$, if the Assumption 2 is hold, the synchronization error system (22) will achieve the multi-switching sliding mode combination synchronization (MSSMCS) via the multi-switching adaptive controller (MSAC) (25) and multi-switching adaptive updating laws (MSAUL) (28). 
Proof. Adopting the Lyapunov function as:

$$
\begin{aligned}
V & =\frac{1}{2} \sum_{p=1}^{n}{ }^{(l w v)} s_{p}^{2}+\frac{1}{2 m_{1}} \sum_{p=1}^{n}\left\{{ }^{(l w v)} \tilde{\rho}_{p}^{T}\right\}\left\{{ }^{(l w v)} \tilde{\rho}_{p}\right\}+\frac{1}{2 m_{2}} \sum_{p=1}^{n}\left\{{ }^{(l w v)} \tilde{\varrho}_{p}^{T}\right\}\left\{{ }^{(l w v)} \tilde{\varrho}_{p}\right\} \\
& +\frac{1}{2} \tilde{\boldsymbol{\theta}}^{T} \boldsymbol{\theta}+\frac{1}{2} \tilde{\boldsymbol{\beta}}^{T} \boldsymbol{\beta}+\frac{1}{2} \tilde{\boldsymbol{\vartheta}}^{T} \boldsymbol{\vartheta} .
\end{aligned}
$$

Taking the $\alpha$ derivative

$$
\begin{aligned}
{ }_{0} D_{t}^{\alpha} V(t, \boldsymbol{x}(t)) \leq & \sum_{p=1}^{n}\left\{{ }^{(l w v)} s_{p}\right\}_{0} D_{t}^{\alpha}\left\{{ }^{(l w v)} s_{p}\right\}+\frac{1}{m_{1}}\left\{\sum_{p=1}^{n}{ }^{(l w v)} \tilde{\rho}_{p}^{T}\right\}_{0} D_{t}^{\alpha}\left\{{ }^{(l w v)} \tilde{\rho_{p}}\right\} \\
& +\frac{1}{m_{2}} \sum_{p=1}^{n}\left\{{ }^{(l w v)} \tilde{\varrho}_{p}^{T}\right\}_{0} D_{t}^{\alpha}\left\{{ }^{(l w v)} \tilde{\varrho}_{p}\right\}+\tilde{\boldsymbol{\theta}}^{T}{ }_{0} D_{t}^{\alpha} \tilde{\boldsymbol{\theta}}+\tilde{\boldsymbol{\beta}}^{T}{ }_{0} D_{t}^{\alpha} \tilde{\boldsymbol{\beta}}+\tilde{\boldsymbol{\vartheta}}^{T}{ }_{0} D_{t}^{\alpha} \tilde{\boldsymbol{\vartheta}} .
\end{aligned}
$$

Then substituting the (27) and MSAUL (28) into Eq. (30), we obtain

$$
\begin{aligned}
{ }_{0} D_{t}^{\alpha} V(t, \boldsymbol{x}(t)) \leq & \sum_{l=1}^{n} \sum_{i=1}^{n}{ }^{(l w v)} s_{i}\left({ }^{(l w v)} \lambda_{i}\right) c_{l i}\left\{-\boldsymbol{H}_{i}(z(t)) \tilde{\boldsymbol{\vartheta}}+\Delta h_{i}(z(t))+\mu_{i}(z, t)\right\} \\
& +\sum_{w=1}^{n} \sum_{j=1}^{m}{ }^{(l w v)} s_{j}\left({ }^{(l w v)} \lambda_{j}\right) b_{w j}\left\{\boldsymbol{G}_{j}(y(t)) \tilde{\boldsymbol{\beta}}+\Delta g_{j}(y(t))+D_{j}(y, t)\right\} \\
& +\sum_{v=1}^{n} \sum_{k=1}^{m}{ }^{(l w v)} s_{k}\left({ }^{(l w v)} \lambda_{k}\right) x_{v k}\left\{\boldsymbol{F}_{k}(x(t)) \tilde{\boldsymbol{\theta}}+\Delta f_{k}(x(t))+d_{k}(x, t)\right\} \\
& -\sum_{p=1}^{n}\left({ }^{(l w v)} \lambda_{p}\right)\left({ }^{(l w v)} \hat{\rho}_{p}+{ }^{(l w v)} \hat{\varrho}_{p}\right) \operatorname{sign}\left({ }^{(l w v)} s_{p}(t)\right) \\
& -\sum_{p=1}^{n}\left({ }^{(l w v)} \lambda_{p}\right) k_{1} t a n h\left({ }^{(l w v)} s_{p}\right)+\tilde{\boldsymbol{\theta}}^{T}\left\{-\boldsymbol{F}^{T}(x(t)) \boldsymbol{\gamma}-\varphi_{1} \operatorname{sign}(\tilde{\boldsymbol{\theta}})|(\tilde{\boldsymbol{\theta}})|^{\omega}\right\} \\
& +\tilde{\boldsymbol{\beta}}^{T}\left\{-\boldsymbol{G}^{T}(y(t)) \boldsymbol{\xi}-\varphi_{2} \operatorname{sign}(\tilde{\boldsymbol{\beta}})|(\tilde{\boldsymbol{\beta}})|^{\omega}\right\} \\
& +\tilde{\boldsymbol{\vartheta}}^{T}\left\{\boldsymbol{H}^{T}(z(t)) \varpi-\varphi_{3} \operatorname{sign}(\tilde{\boldsymbol{\vartheta}})|(\tilde{\boldsymbol{\vartheta}})|^{\omega}\right\} \\
& +\sum_{p=1}^{n}{ }^{(l w v)} \tilde{\rho}_{p}^{T}\left({ }^{(l w v)} \lambda_{p}\right)\left(\left.\right|^{(l w v)} s_{p} \mid\right)+\sum_{p=1}^{(l w v)} \tilde{\varrho}_{p}^{T}\left({ }^{(l w v)} \lambda_{p}\right)\left(\left|{ }^{(l w v)} s_{p}\right|\right) .
\end{aligned}
$$

Since

$$
\begin{aligned}
& \sum_{v=1}^{n} \sum_{k=1}^{m}{ }^{(l w v)} s_{k}\left({ }^{(l w v)} \lambda_{k}\right) x_{v k}\left\{\boldsymbol{F}_{k}(x(t)) \tilde{\boldsymbol{\theta}}\right\}=\tilde{\boldsymbol{\theta}}^{T} \boldsymbol{F}^{T}(x(t)) \boldsymbol{\gamma} \\
& \sum_{w=1}^{n} \sum_{j=1}^{m}{ }^{(l w v)} s_{j}\left({ }^{(l w v)} \lambda_{j}\right) b_{w j}\left\{\boldsymbol{G}_{j}(y(t)) \tilde{\boldsymbol{\beta}}\right\}=\tilde{\boldsymbol{\beta}}^{T} \boldsymbol{G}^{T}(y(t)) \boldsymbol{\xi} \\
& \sum_{l=1}^{n} \sum_{i=1}^{n}{ }^{(l w v)} s_{i}\left({ }^{(l w v)} \lambda_{i}\right) c_{l i}\left\{\boldsymbol{H}_{i}(z(t)) \tilde{\boldsymbol{\vartheta}}\right\}=\tilde{\boldsymbol{\vartheta}}^{T} \boldsymbol{H}^{T}(z(t)) \varpi .
\end{aligned}
$$

Thus

$$
{ }_{0} D_{t}^{\alpha} V(t, \boldsymbol{x}(t)) \leq \sum_{l=1}^{n} \sum_{i=1}^{n}{ }^{(l w v)} s_{i}\left({ }^{(l w v)} \lambda_{i}\right) c_{l i}\left\{\Delta h_{i}(z(t))+\mu_{i}(z, t)\right\}
$$




$$
\begin{aligned}
& +\sum_{w=1}^{n} \sum_{j=1}^{m}{ }^{(l w v)} s_{j}\left({ }^{(l w v)} \lambda_{j}\right) b_{w j}\left\{\Delta g_{j}(y(t))+D_{j}(y, t)\right\} \\
& +\sum_{v=1}^{n} \sum_{k=1}^{m}{ }^{(l w v)} s_{k}\left({ }^{(l w v)} \lambda_{k}\right) x_{v k}\left\{\Delta f_{k}(x(t))+d_{k}(x, t)\right\} \\
& +\tilde{\boldsymbol{\theta}}^{T}\left\{-\boldsymbol{F}^{T}(x(t)) \boldsymbol{\gamma}-\varphi_{1} \operatorname{sign}(\tilde{\boldsymbol{\theta}})|(\tilde{\boldsymbol{\theta}})|^{\omega}\right\} \\
& +\tilde{\boldsymbol{\beta}}^{T}\left\{-\boldsymbol{G}^{T}(y(t)) \boldsymbol{\xi}-\varphi_{2} \operatorname{sign}(\tilde{\boldsymbol{\beta}})|(\tilde{\boldsymbol{\beta}})|^{\omega}\right\} \\
& +\tilde{\boldsymbol{\vartheta}}^{T}\left\{\boldsymbol{H}^{T}(z(t)) \varpi-\varphi_{3} \operatorname{sign}(\tilde{\boldsymbol{\vartheta}})|(\tilde{\boldsymbol{\vartheta}})|^{\omega}\right\}-\sum_{p=1}^{n}\left({ }^{(l w v)} \lambda_{p}\right)\left({ }^{(l w v)} \hat{\rho}_{p}\right. \\
& \left.+{ }^{(l w v)} \hat{\varrho}_{p}\right) \operatorname{sign}\left({ }^{(l w v)} s_{p}(t)\right)+\sum_{p=1}^{n}{ }^{(l w v)} \tilde{\rho}_{p}^{T}\left({ }^{(l w v)} \lambda_{p}\right)\left(\left|{ }^{(l w v)} s_{p}\right|\right) \\
& +\sum_{p=1}^{n}{ }^{(l w v)} \tilde{\varrho}_{p}^{T}\left({ }^{(l w v)} \lambda_{p}\right)\left(\left|{ }^{(l w v)} s_{p}\right|\right)-\sum_{p=1}^{n}\left({ }^{(l w v)} \lambda_{p}\right) k_{1} \tanh \left({ }^{(l w v)} s_{p}\right) \\
& \leq \sum_{p=1}^{n}{ }^{(l w v)} \rho_{p}\left({ }^{(l w v)} \lambda_{p}\right)\left(\left|{ }^{(l w v)} s_{p}\right|\right)+\sum_{p=1}^{n}{ }^{(l w v)} \varrho_{p}\left({ }^{(l w v)} \lambda_{p}\right)\left(\left|{ }^{(l w v)} s_{p}\right|\right) \\
& -\sum_{p=1}^{n}\left({ }^{(l w v)} \lambda_{p}\right)\left({ }^{(l w v)} \hat{\rho}_{p}+{ }^{(l w v)} \hat{\varrho}_{p}\right) \operatorname{sign}\left({ }^{(l w v)} s_{p}(t)\right) \\
& +\sum_{p=1}^{n}{ }^{(l w v)} \tilde{\rho}_{p}^{T}\left({ }^{(l w v)} \lambda_{p}\right)\left(\left.\right|^{(l w v)} s_{p} \mid\right)+\sum_{p=1}^{n}{ }^{(l w v)} \tilde{\varrho}_{p}^{T}\left({ }^{(l w v)} \lambda_{p}\right)\left(\left|{ }^{(l w v)} s_{p}\right|\right) \\
& -\tilde{\boldsymbol{\theta}}^{T}\left(\varphi_{1} \operatorname{sign}(\tilde{\boldsymbol{\theta}})|(\tilde{\boldsymbol{\theta}})|^{\omega}\right)-\tilde{\boldsymbol{\beta}}^{T}\left(\varphi_{2} \operatorname{sign}(\tilde{\boldsymbol{\beta}})|(\tilde{\boldsymbol{\beta}})|^{\omega}\right) \\
& -\tilde{\boldsymbol{\vartheta}}^{T}\left(\varphi_{3} \operatorname{sign}(\tilde{\boldsymbol{\vartheta}})|(\tilde{\boldsymbol{\vartheta}})|^{\omega}\right)-\sum_{p=1}^{n}\left({ }^{(l w v)} \lambda_{p}\right) k_{1} \tanh \left({ }^{(l w v)} s_{p}\right) \\
& =-\tilde{\boldsymbol{\theta}}^{T}\left(\varphi_{1} \operatorname{sign}(\tilde{\boldsymbol{\theta}})|(\tilde{\boldsymbol{\theta}})|^{\omega}\right)-\tilde{\boldsymbol{\beta}}^{T}\left(\varphi_{2} \operatorname{sign}(\tilde{\boldsymbol{\beta}})|(\tilde{\boldsymbol{\beta}})|^{\omega}\right) \\
& -\tilde{\boldsymbol{\vartheta}}^{T}\left(\varphi_{3} \operatorname{sign}(\tilde{\boldsymbol{\vartheta}})|(\tilde{\boldsymbol{\vartheta}})|^{\omega}\right)-\sum_{p=1}^{n}\left({ }^{(l w v)} \lambda_{p}\right) k_{1} \tanh \left({ }^{(l w v)} s_{p}\right) \text {. }
\end{aligned}
$$

Which establishes that the ${ }_{0} D_{t}^{\alpha} V(t, \boldsymbol{x}(t))$ is negative definite. Thus, it follows from the FO Lyapunov stability theory that $\lim _{t \rightarrow \infty}{ }^{(l w v)} e_{p}=0$ is achieved. On the other hand, we can say that the MSSMCS of the drive systems (4), (5) and response system (6) is accomplished in terms of $m \neq n$.

The following corollaries are successfully analyzed from Theorem 2 and their proofs are omitted here. By the way, the Theorem 1 has the same theory, we are not going to describe.

Corollary 1. If the matrices $A \neq 0, B=0, C \neq 0$, then the drive systems (44) achieve the MSSMCS with the response systems (6) provided the following controller,

$$
\begin{aligned}
\sum_{i=1}^{n} c_{l i}\left\{{ }^{(l w v)} u_{p}\right\} & =-\sum_{i=1}^{n} c_{l i} h_{i}+\sum_{k=1}^{m} x_{v k} f_{k}+\sum_{k=1}^{m} x_{v k} \boldsymbol{F}_{k}(x(t)) \hat{\boldsymbol{\theta}}-\sum_{i=1}^{n} c_{l i} \boldsymbol{H}_{i}(z(t)) \hat{\boldsymbol{\vartheta}} \\
& -\left({ }^{(l w v)} \hat{\rho}_{p}+{ }^{(l w v)} \hat{\varrho}_{p}\right) \operatorname{sign}\left({ }^{(l w v)} s_{p}\right)-k_{1} \tanh \left({ }^{(l w v)} s_{p}\right) .
\end{aligned}
$$


And the adaptive updating laws,

$$
\begin{aligned}
& { }_{0} D_{t}^{\alpha} \tilde{\boldsymbol{\theta}}=-\boldsymbol{F}^{T}(x(t)) \boldsymbol{\gamma}-\varphi_{1} \operatorname{sign}(\tilde{\boldsymbol{\theta}})|(\tilde{\boldsymbol{\theta}})|^{w}, \\
& { }_{0} D_{t}^{\alpha} \tilde{\boldsymbol{\vartheta}}=\boldsymbol{H}^{T}(z(t)) \varpi-\varphi_{3} \operatorname{sign}(\tilde{\boldsymbol{\vartheta}})|(\tilde{\boldsymbol{\vartheta}})|^{w}, \\
& { }_{0} D_{t}^{\alpha}\left\{{ }^{(l w v)} \tilde{\rho}_{p}\right\}=\left.m_{1}\left({ }^{(l w v)} \lambda_{p}\right)\right|^{(l w v)} s_{p}(t) \mid, \\
& { }_{0} D_{t}^{\alpha}\left\{{ }^{(l w v)} \tilde{\varrho}_{p}\right\}=\left.m_{2}\left({ }^{(l w v)} \lambda_{p}\right)\right|^{(l w v)} s_{p}(t) \mid,
\end{aligned}
$$

where the explanation of $\gamma, \varpi$ can be seen (28)

Corollary 2. If the matrices $A=0, B \neq 0, C \neq 0$, then the drive systems (5) achieve the MSSMCS with the response systems (6) provided the following controller,

$$
\begin{aligned}
\sum_{i=1}^{n} c_{l i}\left\{{ }^{(l w v)} u_{p}\right\} & =-\sum_{i=1}^{n} c_{l i} h_{i}+\sum_{j=1}^{m} b_{w j} g_{j}+\sum_{j=1}^{m} b_{w j} \boldsymbol{G}_{j}(y(t)) \hat{\boldsymbol{\beta}}-\sum_{i=1}^{n} c_{l i} \boldsymbol{H}_{i}(z(t)) \hat{\boldsymbol{\vartheta}} \\
& -\left({ }^{(l w v)} \hat{\rho}_{p}+{ }^{(l w v)} \hat{\varrho}_{p}\right) \operatorname{sign}\left({ }^{(l w v)} s_{p}\right)-k_{1} \tanh \left({ }^{(l w v)} s_{p}\right) .
\end{aligned}
$$

And the adaptive updating laws,

$$
\begin{aligned}
& { }_{0} D_{t}^{\alpha} \tilde{\boldsymbol{\beta}}=-\boldsymbol{G}^{T}(y(t)) \boldsymbol{\xi}-\varphi_{2} \operatorname{sign}(\tilde{\boldsymbol{\beta}})|(\tilde{\boldsymbol{\beta}})|^{w}, \\
& { }_{0} D_{t}^{\alpha} \tilde{\boldsymbol{\vartheta}}=\boldsymbol{H}^{T}(z(t)) \varpi-\varphi_{3} \operatorname{sign}(\tilde{\boldsymbol{\vartheta}})|(\tilde{\boldsymbol{\vartheta}})|^{w}, \\
& { }_{0} D_{t}^{\alpha}\left\{{ }^{(l w v)} \tilde{\rho}_{p}\right\}=\left.m_{1}\left({ }^{(l w v)} \lambda_{p}\right)\right|^{(l w v)} s_{p}(t) \mid, \\
& { }_{0} D_{t}^{\alpha}\left\{{ }^{(l w v)} \tilde{\varrho}_{p}\right\}=\left.m_{2}\left({ }^{(l w v)} \lambda_{p}\right)\right|^{(l w v)} s_{p}(t) \mid,
\end{aligned}
$$

where the explanation of $\boldsymbol{\xi}, \varpi$ can be seen $(28)$.

Corollary 3. If the matrices $A=0, B=0, C \neq 0$, then the equilibrium point $(0,0,0,0)$ of response systems (66) is asymptotically stable provided the following controller,

$$
\begin{aligned}
\sum_{i=1}^{n} c_{l i}\left\{{ }^{(l w v)} u_{p}\right\} & =-\sum_{i=1}^{n} c_{l i} h_{i}-\sum_{i=1}^{n} c_{l i} \boldsymbol{H}_{i}(z(t)) \hat{\boldsymbol{\vartheta}} \\
& -\left({ }^{(l w v)} \hat{\rho}_{p}+{ }^{(l w v)} \hat{\varrho}_{p}\right) \operatorname{sign}\left({ }^{(l w v)} s_{p}\right)-k_{1} \tanh \left({ }^{(l w v)} s_{p}\right),
\end{aligned}
$$

And the adaptive updating laws,

$$
\begin{aligned}
& { }_{0} D_{t}^{\alpha} \tilde{\boldsymbol{\vartheta}}=\boldsymbol{H}^{T}(z(t)) \varpi-\varphi_{3} \operatorname{sign}(\tilde{\boldsymbol{\vartheta}})|(\tilde{\boldsymbol{\vartheta}})|^{w}, \\
& { }_{0} D_{t}^{\alpha}\left\{{ }^{(l w v)} \tilde{\rho}_{p}\right\}=\left.m_{1}\left({ }^{(l w v)} \lambda_{p}\right)\right|^{(l w v)} s_{p}(t) \mid, \\
& { }_{0} D_{t}^{\alpha}\left\{{ }^{(l w v)} \tilde{\varrho}_{p}\right\}=\left.m_{2}\left({ }^{(l w v)} \lambda_{p}\right)\right|^{(l w v)} s_{p}(t) \mid,
\end{aligned}
$$

where the explanation of $\varpi$ can be seen (28) .

\section{Numerical simulation}

This section mainly demonstrates the reliability and validity of the suggested multi-switching sliding mode combination synchronization scheme. For the D-R systems (4), (5) and (6) with same dimension, we selected two error states to elaborate the method, namely, $i \neq j \neq k$ and $i \neq j=k$. For the D-R systems (44), (5) and (6) with different dimensions, we selected $l \neq w \neq v$ and $l \neq w=v$. In each case, we have given the specific form of controller and parameter adapting rate via the specific FO hyper-chaotic or chaotic systems. . 


\subsection{Numerical simulations for FO chaotic system with same dimension}

As an example we choose FO hyper-chaotic Lorenz, Chen systems as the drive systems, and the FO hyper-chaotic Lü system as the response system. Adding SD to the D-R systems, we obtain

$$
\begin{aligned}
& \left(\begin{array}{l}
{ }_{0} D_{t}^{\alpha} x_{1} \\
{ }_{0} D_{t}^{\alpha} x_{2} \\
{ }_{0} D_{t}^{\alpha} x_{3} \\
{ }_{0} D_{t}^{\alpha} x_{4}
\end{array}\right)=\left(\begin{array}{cccc}
x_{2}-x_{1} & 0 & 0 & 0 \\
0 & x_{1} & 0 & 0 \\
0 & 0 & -x_{3} & 0 \\
0 & 0 & 0 & x_{4}
\end{array}\right)\left(\begin{array}{l}
a_{1} \\
b_{1} \\
c_{1} \\
d_{1}
\end{array}\right)+\left(\begin{array}{c}
x_{4}+\Delta f_{1} \\
-x_{1} x_{3}-x_{2}+\Delta f_{2} \\
x_{1} x_{2}+\Delta f_{3} \\
-x_{2} x_{3}+\Delta f_{4}
\end{array}\right)+\left(\begin{array}{c}
d_{1} \\
d_{2} \\
d_{3} \\
d_{4}
\end{array}\right), \\
& \left(\begin{array}{l}
{ }_{0} D_{t}^{\alpha} y_{1} \\
{ }_{0} D_{t}^{\alpha} y_{2} \\
{ }_{0} D_{t}^{\alpha} y_{3} \\
{ }_{0} D_{t}^{\alpha} y_{4}
\end{array}\right)=\left(\begin{array}{ccccc}
y_{2}-y_{1} & 0 & 0 & 0 & 0 \\
0 & 0 & y_{2} & y_{1} & 0 \\
0 & -y_{3} & 0 & 0 & 0 \\
0 & 0 & 0 & 0 & y_{4}
\end{array}\right)\left(\begin{array}{c}
a_{2} \\
b_{2} \\
c_{2} \\
d_{2} \\
r
\end{array}\right)+\left(\begin{array}{c}
y_{4}+\Delta g_{1} \\
-y_{1} y_{3}+\Delta g_{2} \\
y_{1} y_{2}+\Delta g_{3} \\
y_{2} y_{3}+\Delta g_{4}
\end{array}\right)+\left(\begin{array}{c}
D_{1} \\
D_{2} \\
D_{3} \\
D_{4}
\end{array}\right),(35) \\
& \left(\begin{array}{c}
{ }_{0} D_{t}^{\alpha} z_{1} \\
{ }_{0} D_{t}^{\alpha} z_{2} \\
{ }_{0} D_{t}^{\alpha} z_{3} \\
{ }_{0} D_{t}^{\alpha} z_{4}
\end{array}\right)=\left(\begin{array}{cccc}
z_{2}-z_{1} & 0 & 0 & 0 \\
0 & z_{2} & 0 & 0 \\
0 & 0 & -z_{3} & 0 \\
0 & 0 & 0 & z_{4}
\end{array}\right)\left(\begin{array}{c}
a_{3} \\
b_{3} \\
c_{3} \\
d_{3}
\end{array}\right)+\left(\begin{array}{c}
-z_{1} z_{3}+\Delta h_{2} \\
z_{1}+\Delta h_{2}+\Delta h_{3} \\
z_{1} z_{3}+\Delta h_{4}
\end{array}\right)+\left(\begin{array}{c}
\mu_{1}+u_{1} \\
\mu_{2}+u_{2} \\
\mu_{3}+u_{3} \\
\mu_{4}+u_{4}
\end{array}\right) .
\end{aligned}
$$

Choosing the parameters are $a_{1}=10, b_{1}=28, c_{1}=8 / 3, d_{1}=-1, a_{2}=35, b_{2}=3, c_{2}=$ $12, d_{2}=7, r=0.5, a_{3}=36, b_{3}=20, c_{3}=3, d_{3}=0.5$. The initial conditions take as $x(0)=(2,-2,1,1), y(0)=(1,1,2,2), z(0)=(-20,3,1,3$,$) . When \Delta g=0, \Delta f=0$, $\Delta h=0, d(t)=0, D(t)=0, \mu(t)=0$ and $\alpha=0.97$, we can obtain the attractor graphs of the FO hyper-chaotic Lorenz, Chen, and Lü system using the FO predictioncorrection method which are presented in Fig.1 The multi-switching error state modes between the drive systems (34), (35) and the response system (36) are listed.

$$
\begin{aligned}
& i \neq j \neq k\left\{\begin{array}{llllll}
{ }^{(123)} e_{1}, & { }^{(132)} e_{2}, & { }^{(124)} e_{3}, & { }^{(142)} e_{4}, & { }^{(134)} e_{5}, & { }^{(143)} e_{6}, \\
{ }^{(213)} e_{7}, & { }^{(231)} e_{8}, & { }^{(214)} e_{9}, & { }^{(241)} e_{10}, & { }^{(234)} e_{11}, & { }^{(243)} e_{12}, \\
{ }^{(321)} e_{13}, & { }^{(312)} e_{14}, & { }^{(324)} e_{15}, & { }^{(314)} e_{16}, & { }^{(341)} e_{17}, & { }^{(342)} e_{18}, \\
{ }^{(412)} e_{19}, & { }^{(421)} e_{20}, & { }^{(413)} e_{21}, & { }^{(423)} e_{22}, & { }^{(432)} e_{23}, & { }^{(431)} e_{24} .
\end{array}\right. \\
& i \neq j=k\left\{\begin{array}{llllll}
{ }^{(122)} e_{25}, & { }^{(133)} e_{26}, & { }^{(144)} e_{27}, & { }^{(211)} e_{28}, & { }^{(233)} e_{29}, & { }^{(244)} e_{30}, \\
{ }^{(311)} e_{31}, & { }^{(322)} e_{32}, & { }^{(344)} e_{33}, & { }^{(411)} e_{34}, & { }^{(422)} e_{35}, & { }^{(433)} e_{36}
\end{array}\right. \\
& i=j \neq k\left\{\begin{array}{llllll}
{ }^{(112)} e_{37}, & { }^{(113)} e_{38}, & { }^{(114)} e_{39}, & { }^{(221)} e_{40}, & { }^{(223)} e_{41}, & { }^{(224)} e_{42}, \\
{ }^{(331)} e_{43}, & { }^{(332)} e_{44}, & { }^{(334)} e_{45}, & { }^{(441)} e_{46}, & { }^{(442)} e_{47}, & { }^{(443)} e_{48}
\end{array}\right. \\
& i=k \neq j\left\{\begin{array}{llllll}
{ }^{(121)} e_{49}, & { }^{(131)} e_{50}, & { }^{(141)} e_{51}, & { }^{(212)} e_{52}, & { }^{(232)} e_{53}, & { }^{(242)} e_{54}, \\
{ }^{(313)} e_{55}, & { }^{(323)} e_{56}, & { }^{(343)} e_{57}, & { }^{(414)} e_{58}, & { }^{(424)} e_{59}, & { }^{(434)} e_{60} .
\end{array}\right.
\end{aligned}
$$




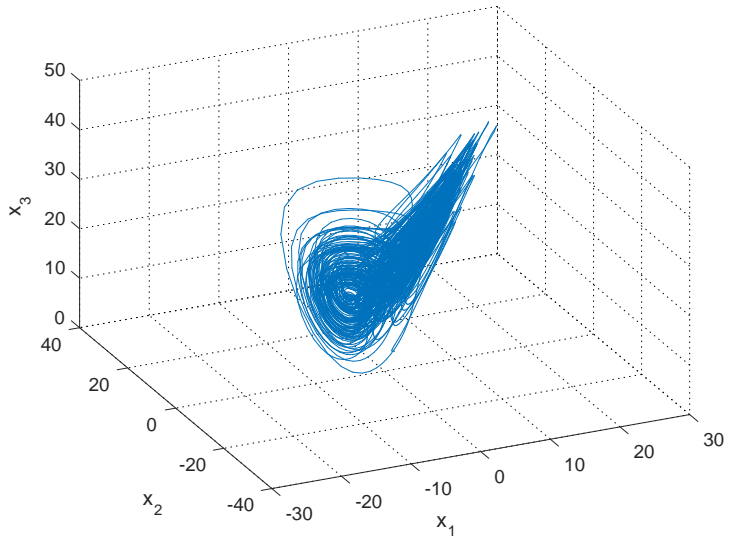

(a)

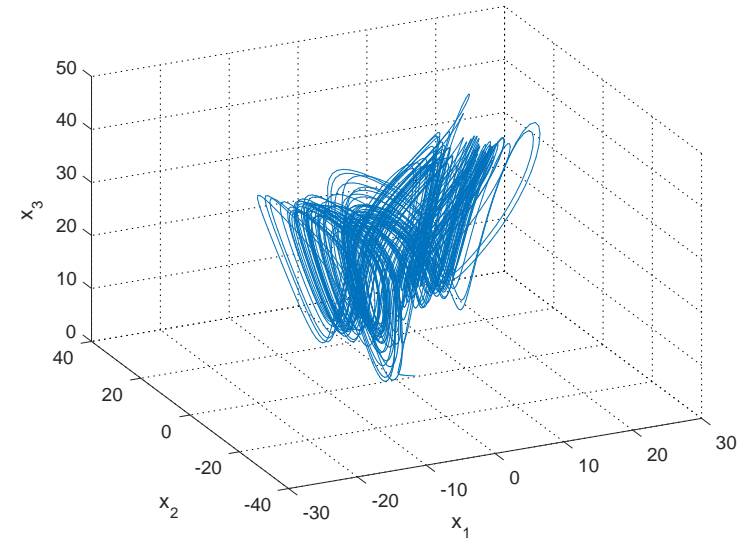

(b)

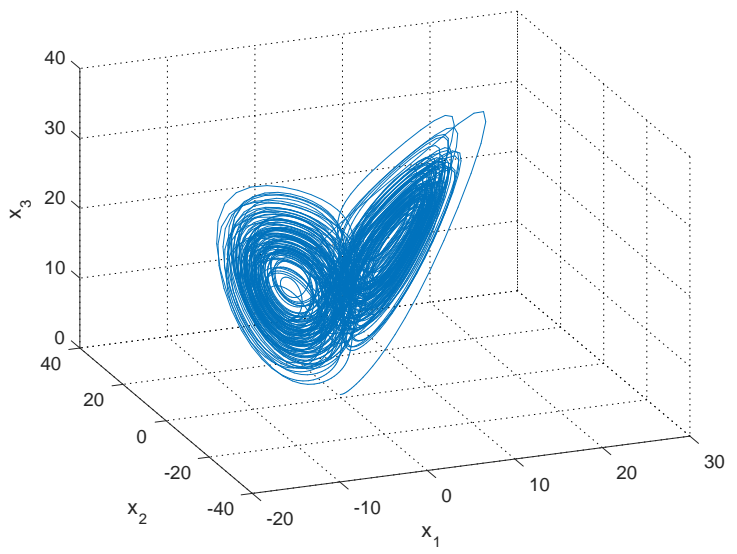

(c)

Fig.1. The 3D phase plot of FO hyper-chaotic Lorenz, Chen, Lü systems indicating in sub-pictures (a)-(c) respectively.

$$
i=k=j \begin{cases}{ }^{(111)} e_{61}, & { }^{(222)} e_{62}, \\ { }^{(333)} e_{63}, & { }^{(444)} e_{64} .\end{cases}
$$

In this chapter, we will select the appropriate switching error variable for the situation of $i \neq j \neq k$, namely, $\boldsymbol{e}=\left({ }^{(124)} e_{3},{ }^{(243)} e_{12},{ }^{(312)} e_{14},{ }^{\left({ }^{(43)}\right.} e_{24}\right)^{T}, i \neq j=k$ namely, $\boldsymbol{e}=$

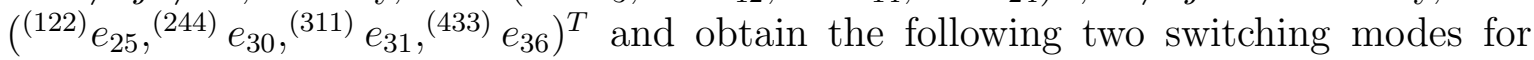
numerical simulation:

$$
\text { Switch-1: }\left(\begin{array}{l}
{ }^{(124)} e_{3}=c_{11} z_{1}-\left(b_{22} y_{2}+x_{44} x_{4}\right) \\
{ }^{(243)} e_{12}=c_{22} z_{2}-\left(b_{44} y_{4}+x_{33} x_{3}\right) \\
{ }^{(312)} e_{14}=c_{33} z_{3}-\left(b_{11} y_{1}+x_{22} x_{2}\right) \\
{ }^{(431)} e_{24}=c_{44} z_{4}-\left(b_{33} y_{3}+x_{11} x_{1}\right)
\end{array}\right)
$$




$$
\text { Switch-2: }\left(\begin{array}{l}
{ }^{(122)} e_{25}=c_{11} z_{1}-\left(b_{22} y_{2}+x_{22} x_{2}\right) \\
{ }^{(244)} e_{30}=c_{22} z_{2}-\left(b_{44} y_{4}+x_{44} x_{4}\right) \\
{ }^{(311)} e_{31}=c_{33} z_{3}-\left(b_{11} y_{1}+x_{11} x_{1}\right) \\
{ }^{\left({ }^{433)}\right.} e_{36}=c_{44} z_{4}-\left(b_{33} y_{3}+x_{33} x_{3}\right)
\end{array}\right)
$$

In order to confirm the robustness and reliability of the investigated MSAC (13) and MSAUL (16), the nonlinear uncertainty and external disturbance are selected as follows:

$$
\begin{aligned}
\boldsymbol{d}(\boldsymbol{x}(t)) & =(-0.1 \cos (t),-0.2 \cos (2 t), 0.3 \sin (3 t), 0.4 \sin (4 t))^{T}, \\
\boldsymbol{D}(\boldsymbol{y}(t)) & =(-0.1 \sin (t),-0.2 \sin (2 t), 0.3 \cos (3 t), 0.4 \cos (4 t))^{T}, \\
\boldsymbol{\mu}(\boldsymbol{z}(t)) & =(0.1 \cos (5 t), 0.2 \cos (6 t), 0.3 \sin (7 t), 0.4 \sin (8 t))^{T}, \\
\Delta \boldsymbol{f}(\boldsymbol{x}(t)) & =\left(0.1 \cos \left(2 t x_{1}\right), 0.2 \cos \left(2 t x_{2}\right), 0.3 \sin \left(2 t x_{3}\right), 0.4 \sin \left(2 t x_{4}\right)\right)^{T}, \\
\Delta \boldsymbol{g}(\boldsymbol{y}(t)) & =\left(0.1 \sin (2 \pi t), 0.2 \sin \left(\operatorname{sign}\left(y_{2}\right)\right), 0.3 \cos \left(2 \pi y_{3}\right), 0.4 \cos \left(4 t x_{8}\right)\right)^{T}, \\
\Delta \boldsymbol{h}(\boldsymbol{z}(t)) & =\left(0.1 \sin \left(2 t \operatorname{sign}\left(z_{1}\right)\right), 0.2 \sin \left(2 t \operatorname{sign}\left(z_{2}\right)\right), 0.3 \sin (3 t), 0.4 \sin (4 t)^{T} .\right.
\end{aligned}
$$

\subsubsection{Switch-1}

It follows from switch-1 (37) that the FO error dynamic system is expressed as:

$$
\begin{aligned}
{ }_{0} D_{t}^{\alpha}\left\{{ }^{(124)} e_{3}\right\} & =c_{11}\left\{{ }_{0} D_{t}^{\alpha} z_{1}\right\}-b_{22}\left\{{ }_{0} D_{t}^{\alpha} y_{2}\right\}-a_{44}\left\{{ }_{0} D_{t}^{\alpha} x_{4}\right\} \\
& =c_{11}\left\{\left(z_{2}-z_{1}\right) a_{3}+z_{4}+\Delta h_{1}+\mu_{1}+{ }^{(124)} u_{3}\right\} \\
& -b_{22}\left\{y_{2} c_{2}+d_{2} y_{1}-y_{1} y_{3}+\Delta g_{2}+D_{2}\right\} \\
& -a_{44}\left\{x_{4} d_{1}-x_{2} x_{3}+\Delta f_{4}+d_{4}\right\}, \\
{ }_{0} D_{t}^{\alpha}\left\{{ }^{(243)} e_{12}\right\} & =c_{22}\left\{{ }_{0} D_{t}^{\alpha} z_{2}\right\}-b_{44}\left\{{ }_{0} D_{t}^{\alpha} y_{4}\right\}-a_{33}\left\{{ }_{0} D_{t}^{\alpha} x_{3}\right\} \\
& =c_{22}\left\{z_{2} b_{3}-z_{1} z_{3}+\Delta h_{2}+\mu_{2}+{ }^{(243)} u_{12}\right\} \\
& -b_{44}\left\{y_{4} r+y_{2} y_{3}+\Delta g_{4}+D_{4}\right\} \\
& -a_{33}\left\{-x_{3} c_{1}+x_{1} x_{2}+\Delta f_{3}+d_{3}\right\}, \\
{ }_{0} D_{t}^{\alpha}\left\{{ }^{(312)} e_{14}\right\} & =c_{33}\left\{{ }_{0} D_{t}^{\alpha} z_{3}\right\}-b_{11}\left\{{ }_{0} D_{t}^{\alpha} y_{1}\right\}-a_{22}\left\{{ }_{0} D_{t}^{\alpha} x_{2}\right\} \\
& =c_{33}\left\{-z_{3} c_{3}+z_{1} z_{2}+\Delta h_{3}+\mu_{3}+{ }^{(312)} u_{14}\right\} \\
& -b_{11}\left\{\left(y_{2}-y_{1}\right) a_{2}+y_{4}+\Delta g_{1}+D_{1}\right\} \\
& -a_{22}\left\{x_{1} b_{1}-x_{1} x_{3}-x_{2}+\Delta f_{2}+d_{2}\right\}, \\
{ }_{0} D_{t}^{\alpha}\left\{{ }^{(431)} e_{24}\right\} & =c_{44}\left\{{ }_{0} D_{t}^{\alpha} z_{4}\right\}-b_{33}\left\{{ }_{0} D_{t}^{\alpha} y_{3}\right\}-a_{11}\left\{{ }_{0} D_{t}^{\alpha} x_{1}\right\} \\
& =c_{44}\left\{z_{4} d_{3}+z_{1} z_{3}+\Delta h_{4}+\mu_{4}+{ }^{(431)} u_{24}\right\} \\
& -b_{33}\left\{\left(-y_{3} b_{2}+y_{1} y_{2}+\Delta g_{3}+D_{3}\right\}\right. \\
& -a_{11}\left\{\left(x_{2}-x_{1}\right) a_{1}+x_{4}+\Delta f_{1}+d_{1}\right\} .
\end{aligned}
$$

It follows from the form of MSAC (13) and MSAUL (16) that the controller is designed as follows:

$$
\begin{aligned}
{ }^{(124)} u_{3} & =-z_{4}-\left(z_{2}-z_{1}\right) \hat{a}_{3}+\frac{b_{22}}{c_{11}}\left(-y_{1} y_{3}+y_{2} \hat{c}_{2}+\hat{d}_{2} y_{1}\right)+\frac{a_{44}}{c_{11}}\left(-x_{2} x_{3}+x_{4} \hat{d}_{1}\right) \\
& -\left({ }^{(124)} \hat{r}_{3}+{ }^{(124)} \hat{q}_{3}\right) \operatorname{sign}\left({ }^{(124)} s_{3}\right)-k_{1} \tanh \left({ }^{(124)} s_{3}\right),
\end{aligned}
$$




$$
\begin{aligned}
{ }^{(243)} u_{12} & =z_{1} z_{3}-z_{2} \hat{b}_{3}+\frac{b_{44}}{c_{22}}\left(y_{2} y_{3}+y_{4} \hat{r}\right)+\frac{a_{33}}{c_{22}}\left(x_{2} x_{1}-x_{3} \hat{c}_{1}\right) \\
& -\left({ }^{(243)} \hat{r}_{12}+{ }^{(243)} \hat{q}_{12}\right) \operatorname{sign}\left({ }^{(243)} s_{12}\right)-k_{1} \tanh \left({ }^{(243)} s_{12}\right), \\
{ }^{(312)} u_{14} & =-z_{1} z_{2}+z_{3} \hat{c}_{3}+\frac{b_{11}}{c_{33}}\left(y_{4}+\left(y_{2}-y_{1}\right) \hat{a}_{2}\right)+\frac{a_{22}}{c_{33}}\left(x_{1} x_{3}+x_{2}+x_{1} \hat{b}_{1}\right) \\
& -\left({ }^{(312)} \hat{r}_{14}+{ }^{(312)} \hat{q}_{14}\right) \operatorname{sign}\left({ }^{(312)} s_{14}\right)-k_{1} \tanh \left({ }^{(312)} s_{14}\right), \\
{ }^{(431)} u_{24} & =-z_{1} z_{3}-z_{4} \hat{d}_{3}+\frac{b_{33}}{c_{44}}\left(y_{1} y_{2}-y_{3} \hat{b}_{2}\right)+\frac{a_{11}}{c_{44}}\left(x_{4}+\left(x_{2}-x_{1}\right) \hat{a}_{1}\right) \\
& -\left({ }^{(431)} \hat{r}_{24}+{ }^{(431)} \hat{q}_{24}\right) \operatorname{sign}\left({ }^{(431)} s_{24}\right)-k_{1} \tanh \left({ }^{(431)} s_{24}\right) .
\end{aligned}
$$

and the parameters updating laws are designed as follows:

$$
\begin{array}{ll}
\hat{a}_{1}=-\left(x_{2}-x_{1}\right)\left\{{ }^{(431)} s_{24}\right\}-k_{2} \operatorname{sign}\left(\tilde{a}_{1}\right)\left|\tilde{a}_{1}\right|^{\omega}, & \hat{b}_{1}=-x_{1}\left\{{ }^{(312)} s_{14}\right\}-k_{2} \operatorname{sign}\left(\tilde{b}_{1}\right)\left|\tilde{b}_{1}\right|^{\omega}, \\
\hat{c}_{1}=x_{3}\left\{{ }^{(243)} s_{12}\right\}-k_{2} \operatorname{sign}\left(\tilde{c}_{1}\right)\left|\tilde{c}_{1}\right|^{\omega}, & \hat{d}_{1}=-x_{4}\left\{{ }^{(124)} s_{3}\right\}-k_{2} \operatorname{sign}\left(\tilde{d}_{1}\right)\left|\tilde{d}_{1}\right|^{\omega}, \\
\hat{a}_{2}=-\left(y_{2}-y_{1}\right)\left\{{ }^{\left({ }^{2} 2\right)} s_{14}\right\}-k_{3} \operatorname{sign}\left(\tilde{a}_{2}\right)\left|\tilde{a}_{2}\right|^{\omega}, & \hat{b}_{2}=y_{3}\left\{{ }^{(431)} s_{24}\right\}-k_{3} \operatorname{sign}\left(\tilde{b}_{2}\right)\left|\tilde{b}_{2}\right|^{\omega}, \\
\hat{c}_{2}=-y_{2}\left\{{ }^{(124)} s_{3}\right\}-k_{3} \operatorname{sign}\left(\tilde{c}_{2}\right)\left|\tilde{c}_{2}\right|^{\omega}, & \hat{d}_{2}=-y_{1}\left\{{ }^{(124)} s_{3}\right\}-k_{3} \operatorname{sign}\left(\tilde{d}_{2}\right)\left|\tilde{d}_{2}\right|^{\omega}, \\
\hat{r}=-y_{4}\left\{{ }^{(243)} s_{12}\right\}-k_{3} \operatorname{sign}(\tilde{r}) \mid \tilde{r}^{\omega}, & \hat{a}_{3}=\left(z_{2}-z_{1}\right)\left\{{ }^{(124)} s_{3}\right\}-k_{4} \operatorname{sign}\left(\tilde{a}_{3}\right)\left|\tilde{a}_{3}\right|^{\omega}, \\
\hat{b}_{3}=z_{2}\left\{{ }^{(243)} s_{12}\right\}-k_{4} \operatorname{sign}\left(\tilde{b}_{3}\right)\left|\tilde{b}_{3}\right|^{\omega}, & \hat{c}_{3}=-z_{3}\left\{{ }^{(312)} s_{14}\right\}-k_{4} \operatorname{sign}\left(\tilde{c}_{3}\right)\left|\tilde{c}_{3}\right|{ }^{\omega}, \\
\hat{d}_{3}=z_{4}\left\{{ }^{(431)} s_{24}\right\}-k_{4} \operatorname{sign}\left(\tilde{d}_{3}\right)\left|\tilde{d}_{3}\right|^{\omega}, & { }^{(124)} \hat{r}_{3}=\left.m_{1}\left({ }^{(124)} \lambda_{3}\right)\right|^{(124)} s_{3} \mid, \\
{ }^{(243)} \hat{r}_{12}=\left.m_{1}\left\{{ }^{(243)} \lambda_{12}\right\}\right|^{(243)} s_{12} \mid, & { }^{(312)} \hat{r}_{14}=\left.m_{1}\left\{{ }^{(312)} \lambda_{14}\right\}\right|^{\left({ }^{(312)}\right.} s_{14} \mid, \\
{ }^{(431)} \hat{r}_{24}=\left.m_{1}\left\{{ }^{(431)} \lambda_{24}\right\}\right|^{(431)} s_{24} \mid, & { }^{(124)} \hat{q}_{3}=\left.m_{1}\left\{{ }^{(124)} \lambda_{3}\right\}\right|^{(124)} s_{3} \mid, \\
{ }^{(243)} \hat{q}_{12}=\left.m_{2}\left\{{ }^{(243)} \lambda_{12}\right\}\right|^{(243)} s_{12} \mid, & { }^{(312)} \hat{q}_{14}=\left.m_{2}\left\{{ }^{(312)} \lambda_{14}\right\}\right|^{(312)} s_{14} \mid, \\
{ }^{(431)} \hat{q}_{24}=\left.m_{2}\left\{{ }^{(431)} \lambda_{24}\right\}\right|^{(431)} s_{24} \mid . &
\end{array}
$$

\subsubsection{Switch-2}

It follows from switch-2 (38) that the FO error dynamic system is expressed as:

$$
\begin{aligned}
{ }_{0} D_{t}^{\alpha}\left\{{ }^{(122)} e_{25}\right\} & =c_{11}\left\{{ }_{0} D_{t}^{\alpha} z_{1}\right\}-b_{22}\left\{{ }_{0} D_{t}^{\alpha} y_{2}\right\}-a_{220}\left\{D_{t}^{\alpha} x_{2}\right\} \\
= & c_{11}\left\{\left(z_{2}-z_{1}\right) a_{3}+z_{4}+\Delta h_{1}+\mu_{1}+{ }^{(122)} u_{25}\right\} \\
& -b_{22}\left\{y_{2} c_{2}+d_{2} y_{1}-y_{1} y_{3}+\Delta g_{2}+D_{2}\right\} \\
& -a_{22}\left\{x_{1} b_{1}-x_{1} x_{3}-x_{2}+\Delta f_{2}+d_{2}\right\} \\
{ }_{0} D_{t}^{\alpha}\left\{{ }^{(244)} e_{30}\right\} & =c_{22}\left\{{ }_{0} D_{t}^{\alpha} z_{2}\right\}-b_{44}\left\{{ }_{0} D_{t}^{\alpha} y_{4}\right\}-a_{44}\left\{{ }_{0} D_{t}^{\alpha} x_{4}\right\} \\
& =c_{22}\left\{z_{2} b_{3}-z_{1} z_{3}+\Delta h_{2}+\mu_{2}+{ }^{(244)} u_{30}\right\} \\
& -b_{44}\left\{y_{4} r+y_{2} y_{3}+\Delta g_{4}+D_{4}\right\} \\
& -a_{44}\left\{x_{4} d_{1}-x_{2} x_{3}+\Delta f_{4}+d_{4}\right\} \\
{ }_{0} D_{t}^{\alpha}\left\{{ }^{(311)} e_{31}\right\} & =c_{33}\left\{{ }_{0} D_{t}^{\alpha} z_{3}\right\}-b_{11}\left\{{ }_{0} D_{t}^{\alpha} y_{1}\right\}-a_{11}\left\{{ }_{0} D_{t}^{\alpha} x_{1}\right\} \\
& =c_{33}\left\{-z_{3} c_{3}+z_{1} z_{2}+\Delta h_{3}+\mu_{3}+{ }^{(311)} u_{31}\right\} \\
& -b_{11}\left\{\left(y_{2}-y_{1}\right) a_{2}+y_{4}+\Delta g_{1}+D_{1}\right\} \\
& -a_{11}\left\{\left(x_{2}-x_{1}\right) a_{1}+x_{4}+\Delta f_{1}+d_{1}\right\} \\
{ }_{0} D_{t}^{\alpha}\left\{{ }^{(433)} e_{36}\right\} & =c_{44}\left\{{ }_{0} D_{t}^{\alpha} z_{4}\right\}-b_{33}\left\{{ }_{0} D_{t}^{\alpha} y_{3}\right\}-a_{33}\left\{{ }_{0} D_{t}^{\alpha} x_{3}\right\} \\
& =c_{44}\left\{z_{4} d_{3}+z_{1} z_{3}+\Delta h_{4}+\mu_{4}+{ }^{(433)} u_{36}\right\}
\end{aligned}
$$




$$
\begin{aligned}
& -b_{33}\left\{-y_{3} b_{2}+y_{1} y_{2}+\Delta g_{3}+D_{3}\right\} \\
& -a_{33}\left\{-x_{3} c_{1}+x_{1} x_{2}+\Delta f_{3}+d_{3}\right\}
\end{aligned}
$$

It follows from the form of MSAC (13) and MSAUL (16) that the controller is designed as follows:

$$
\begin{aligned}
{ }^{(122)} u_{25} & =-z_{4}-\left(z_{2}-z_{1}\right) \hat{a}_{3}+\frac{b_{22}}{c_{11}}\left(y_{1} y_{3}+y_{2} \hat{c}_{2}+\hat{d}_{2} y_{1}\right)+\frac{a_{22}}{c_{11}}\left(-x_{1} x_{3}-x_{2}+x_{1} \hat{b}_{1}\right) \\
& -\left({ }^{(122)} \hat{r}_{25}+{ }^{(122)} \hat{q}_{25}\right) \operatorname{sign}\left({ }^{(122)} s_{25}\right)-k_{1} \tanh \left({ }^{(122)} s_{25}\right), \\
{ }^{(244)} u_{30}= & z_{1} z_{3}-z_{2} \hat{b}_{3}+\frac{b_{44}}{c_{22}}\left(y_{2} y_{3}+y_{4} \hat{r}\right)+\frac{a_{44}}{c_{22}}\left(-x_{2} x_{3}+x_{4} \hat{d}_{1}\right) \\
& -\left({ }^{(244)} \hat{r}_{30}+{ }^{(244)} \hat{q}_{30}\right) \operatorname{sign}\left({ }^{(244)} s_{30}\right)-k_{1} \tanh \left({ }^{(244)} s_{30}\right), \\
{ }^{(311)} u_{31}= & -z_{1} z_{2}+z_{3} \hat{c}_{3}+\frac{b_{11}}{c_{33}}\left(y_{4}+\left(y_{2}-y_{1}\right) \hat{a}_{2}\right)+\frac{a_{11}}{c_{33}}\left(x_{4}+\left(x_{2}-x_{1}\right) \hat{a}_{1}\right) \\
& -\left({ }^{(311)} \hat{r}_{31}+{ }^{\left({ }^{(111)}\right.} \hat{q}_{31}\right) \operatorname{sign}\left({ }^{(311)} s_{31}\right)-k_{1} \tanh \left({ }^{(311)} s_{31}\right), \\
{ }^{(433)} u_{36}= & -z_{1} z_{3}-z_{4} \hat{d}_{3}+\frac{b_{33}}{c_{44}}\left(y_{1} y_{2}-y_{3} \hat{b}_{2}\right)+\frac{a_{33}}{c_{44}}\left(x_{1} x_{2}-x_{3} \hat{c}_{1}\right) \\
& -\left({ }^{(433)} \hat{r}_{36}+{ }^{(433)} \hat{q}_{36}\right) \operatorname{sign}\left({ }^{(433)} s_{36}\right)-k_{1} \tanh \left({ }^{(433)} s_{36}\right) .
\end{aligned}
$$

and the parameters updating laws are designed as follows:

$$
\begin{array}{ll}
\left.\hat{a}_{1}=-\left(x_{2}-x_{1}\right){ }^{\left({ }^{(311)}\right.} s_{31}\right)-k_{2} \operatorname{sign}\left(\tilde{a}_{1}\right)\left|\tilde{a}_{1}\right|^{\omega}, & \hat{b}_{1}=-x_{1}\left({ }^{(122)} s_{25}\right)-k_{2} \operatorname{sign}\left(\tilde{b}_{1}\right)\left|\tilde{b}_{1}\right|^{\omega}, \\
\hat{c}_{1}=x_{3}\left({ }^{(433)} s_{36}\right)-k_{2} \operatorname{sign}\left(\tilde{c}_{1}\right)\left|\tilde{c}_{1}\right|^{\omega}, & \hat{d}_{1}=-x_{4}\left(\left({ }^{(244)} s_{30}\right)\right)-k_{2} \operatorname{sign}\left(\tilde{d}_{1}\right)\left|\tilde{d}_{1}\right|^{\omega}, \\
\hat{a}_{2}=-\left(y_{2}-y_{1}\right)\left({ }^{(311)} s_{31}\right)-k_{3} \operatorname{sign}\left(\tilde{a}_{2}\right)\left|\tilde{a}_{2}\right|^{\omega}, & b_{2}=y_{3}\left({ }^{(433)} s_{36}\right)-k_{3} \operatorname{sign}\left(\tilde{b}_{2}\right)\left|\tilde{b}_{2}\right|^{\omega}, \\
\hat{c}_{2}=-y_{2}\left({ }^{(122)} s_{25}\right)-k_{3} \operatorname{sign}\left(\tilde{c}_{2}\right)\left|\tilde{c}_{2}\right|^{\omega}, & \hat{d}_{2}=-y_{1}\left({ }^{(122)} s_{25}\right)-k_{3} \operatorname{sign}\left(\tilde{d}_{2}\right)\left|\tilde{d}_{2}\right|{ }^{\omega}, \\
\hat{r}=-y_{4}\left({ }^{(244)} s_{30}\right)-k_{3} \operatorname{sign}(\tilde{r})|\tilde{r}|^{\omega}, & \hat{a}_{3}=\left(z_{2}-z_{1}\right)\left({ }^{(122)} s_{25}\right)-k_{4} \operatorname{sign}\left(\tilde{a}_{3}\right)\left|\tilde{a}_{3}\right|^{\omega}, \\
\hat{b}_{3}=z_{2}\left({ }^{(244)} s_{30}\right)-k_{4} \operatorname{sign}\left(\tilde{b}_{3}\right)\left|\tilde{b}_{3}\right|^{\omega}, & \hat{c}_{3}=-z_{3}\left({ }^{(311)} s_{31}\right)-k_{4} \operatorname{sign}\left(\tilde{c}_{3}\right)\left|\tilde{c}_{3}\right|^{\omega}, \\
\hat{d}_{3}=z_{4}\left({ }^{(433)} s_{36}\right)-k_{4} \operatorname{sign}\left(\tilde{d}_{3}\right)\left|\tilde{d}_{3}\right|^{\omega}, & { }^{(122)} \hat{r}_{25}=\left.m_{1}\left({ }^{(122)} \lambda_{25}\right)\right|^{(122)} s_{25} \mid, \\
{ }^{(244)} \hat{r}_{30}=\left.m_{1}\left\{{ }^{(244)} \lambda_{30}\right\}\right|^{(244)} s_{30} \mid, & { }^{(311)} \hat{r}_{31}=\left.m_{1}\left\{{ }^{(311)} \lambda_{31}\right\}\right|^{(311)} s_{31} \mid, \\
{ }^{(433)} \hat{r}_{36}=\left.m_{1}\left\{{ }^{(433)} \lambda_{36}\right\}\right|^{(433)} s_{36} \mid, & { }^{(122)} \hat{q}_{25}=\left.m_{2}\left({ }^{(122)} \lambda_{25}\right)\right|^{(122)} s_{25} \mid, \\
{ }^{(244)} \hat{q}_{30}=\left.m_{2}\left\{{ }^{(244)} \lambda_{30}\right\}\right|^{(244)} s_{30} \mid, & { }^{(311)} \hat{q}_{31}=\left.m_{2}\left\{{ }^{(311)} \lambda_{31}\right\}\right|^{(311)} s_{31} \mid, \\
{ }^{(433)} \hat{q}_{36}=\left.m_{2}\left\{{ }^{(433)} \lambda_{36}\right\}\right|^{(433)} s_{36} \mid . &
\end{array}
$$

In this two numerical simulations, we adopt the matrices $A, B, C$ as identity matrices. The initial values of the drive systems (34), (35) and response system (36) are selected as $x(0)=(2,-2,1,1), y(0)=(1,1,2,2), z(0)=(-20,3,1,3)$. The initial conditions of the unknown parameter in D-R system and upper bound of SD are selected as $\left(a_{1}(0), b_{1}(0), c_{1}(0), d_{1}(0)\right)=(2,1,-2,1),\left(a_{2}(0), b_{2}(0), c_{2}(0), d_{2}(0), r(0)\right)=$ $(1,-3,1,1,-5),\left(a_{3}(0), b_{3}(0), c_{3}(0), d_{3}(0)\right)=(1,1,-2,-2),\left(r_{1}(0), r_{2}(0), r_{3}(0), r_{4}(0)\right)=$ $(-2.5,1,-3,1),\left(q_{1}(0), q_{2}(0), q_{3}(0), q_{4}(0)\right)=(1,-2.5,-5,1)$. The constants ${ }^{(i j k)} \lambda_{p}=$ $2, m_{1}=10, m_{2}=10, k_{1}=10, k_{2}=10, k_{3}=10, k_{4}=10, \omega=0.5$. The trajectories about the error variables $\boldsymbol{e}=\left({ }^{(124)} e_{3},{ }^{(243)} e_{12},{ }^{(312)} e_{14},{ }^{\left({ }^{(431)}\right.} e_{24}\right)^{T}$ and $\boldsymbol{e}=$

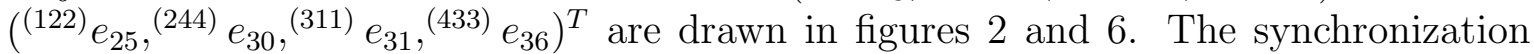
for the state trajectories of drive systems (34), (35) and response system (36) are drawn in figures 3 and 7 . The trajectories of estimations $\hat{\boldsymbol{\theta}}, \hat{\boldsymbol{\beta}}, \hat{\boldsymbol{\vartheta}}$ are drawn in figures 4 and 8 , ${ }^{(i j k)} r_{p}$, and ${ }^{(i j k)} q_{p}$ are drawn in figures 5 and 9 . Motivated by the numerical simulation 


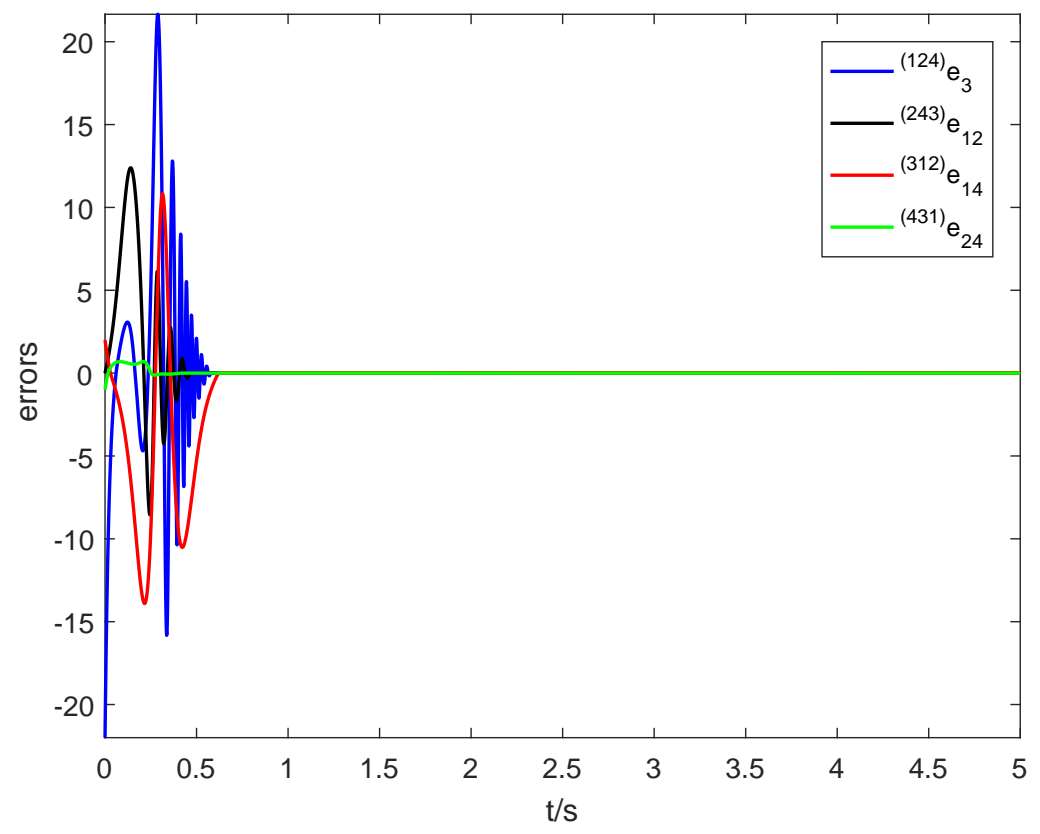

Fig.2. The synchronization errors ${ }^{(124)} e_{3},{ }^{(243)} e_{12},{ }^{(312)} e_{14},{ }^{(431)} e_{24}$ change with time $t$.

results, it can reveal that the drive systems (44), (5) and response system (6) achieve MSSMCS. Therefore, the multi-switching adaptive controllers (MSAC) (13) and some suitable multi-switching adaptive updating laws (MSAUL) (16) are effective.

\subsection{Numerical simulations for FO chaotic system with d- iffrenrt dimensions}

As an example we choose FO Lorenz, Chen chaotic system as the drive system, and the FO hyper-chaotic Lü system as the response system. Adding SD to the D-R systems, we obtain

$$
\begin{aligned}
& \left(\begin{array}{c}
{ }_{0} D_{t}^{\alpha} x_{1} \\
{ }_{0} D_{t}^{\alpha} x_{2} \\
{ }_{0} D_{t}^{\alpha} x_{3}
\end{array}\right)=\left(\begin{array}{ccc}
x_{2}-x_{1} & 0 & 0 \\
0 & x_{1} & 0 \\
0 & 0 & -x_{3}
\end{array}\right)\left(\begin{array}{l}
a_{1} \\
b_{1} \\
c_{1}
\end{array}\right)+\left(\begin{array}{c}
0+\Delta f_{1} \\
-x_{1} x_{3}-x_{2}+\Delta f_{2} \\
x_{1} x_{2}+\Delta f_{3}
\end{array}\right)+\left(\begin{array}{c}
d_{1} \\
d_{2} \\
d_{3}
\end{array}\right),(40) \\
& \left(\begin{array}{c}
{ }_{0} D_{t}^{\alpha} y_{1} \\
{ }_{0} D_{t}^{\alpha} y_{2} \\
{ }_{0} D_{t}^{\alpha} y_{3}
\end{array}\right)=\left(\begin{array}{ccc}
y_{2}-y_{1} & 0 & 0 \\
-y_{1} & 0 & y_{1}+y_{2} \\
0 & -y_{3} & 0
\end{array}\right)\left(\begin{array}{l}
a_{2} \\
b_{2} \\
c_{2}
\end{array}\right)+\left(\begin{array}{c}
0+\Delta g_{1} \\
-y_{1} y_{3}+\Delta g_{2} \\
y_{1} y_{2}+\Delta g_{3}
\end{array}\right)+\left(\begin{array}{c}
D_{1} \\
D_{2} \\
D_{3}
\end{array}\right),(41) \\
& \left(\begin{array}{c}
{ }_{0} D_{t}^{\alpha} z_{1} \\
{ }_{0} D_{t}^{\alpha} z_{2} \\
{ }_{0} D_{t}^{\alpha} z_{3} \\
{ }_{0} D_{t}^{\alpha} z_{4}
\end{array}\right)=\left(\begin{array}{cccc}
z_{2}-z_{1} & 0 & 0 & 0 \\
0 & z_{2} & 0 & 0 \\
0 & 0 & -z_{3} & 0 \\
0 & 0 & 0 & z_{4}
\end{array}\right)\left(\begin{array}{l}
a_{3} \\
b_{3} \\
c_{3} \\
d_{3}
\end{array}\right)+\left(\begin{array}{c}
z_{4}+\Delta h_{1} \\
-z_{1} z_{3}+\Delta h_{2} \\
z_{1} z_{2}+\Delta h_{3} \\
z_{1} z_{3}+\Delta h_{4}
\end{array}\right)+\left(\begin{array}{c}
\mu_{1}+u_{1} \\
\mu_{2}+u_{2} \\
\mu_{3}+u_{3} \\
\mu_{4}+u_{4}
\end{array}\right),
\end{aligned}
$$

Choosing the parameters are $a_{1}=10, b_{1}=28, c_{1}=8 / 3, a_{2}=35, b_{2}=3, c_{2}=28, a_{3}=$ $36, b_{3}=20, c_{3}=3, d_{3}=0.5$. The initial conditions take as $x(0)=(2,-2,1), y(0)=$ 


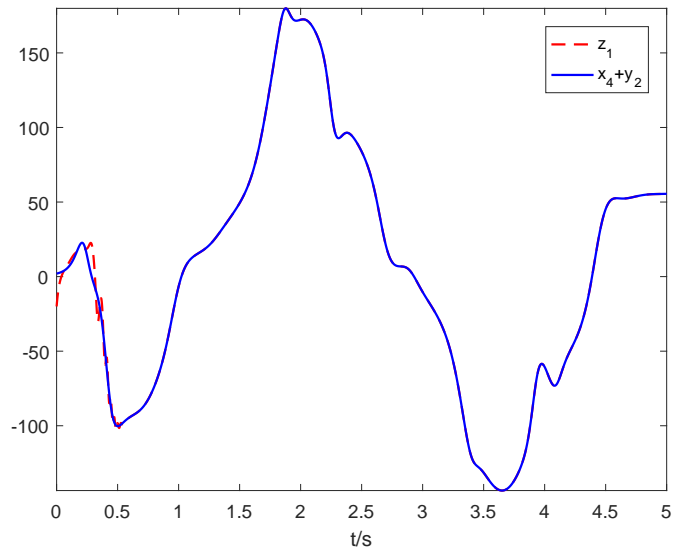

(a)

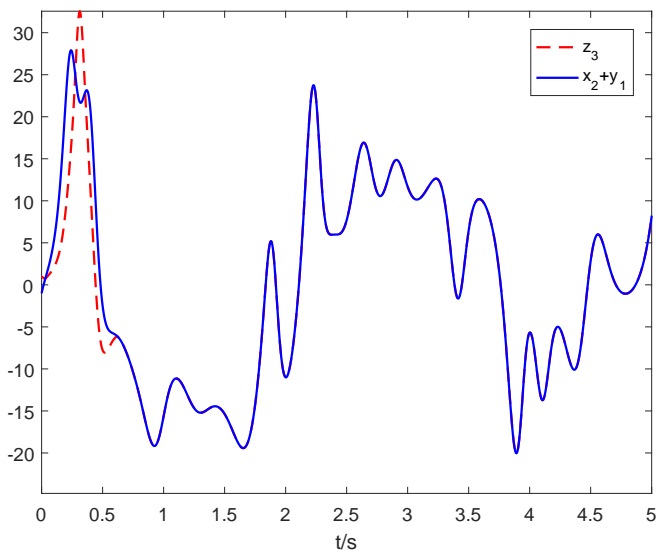

(c)

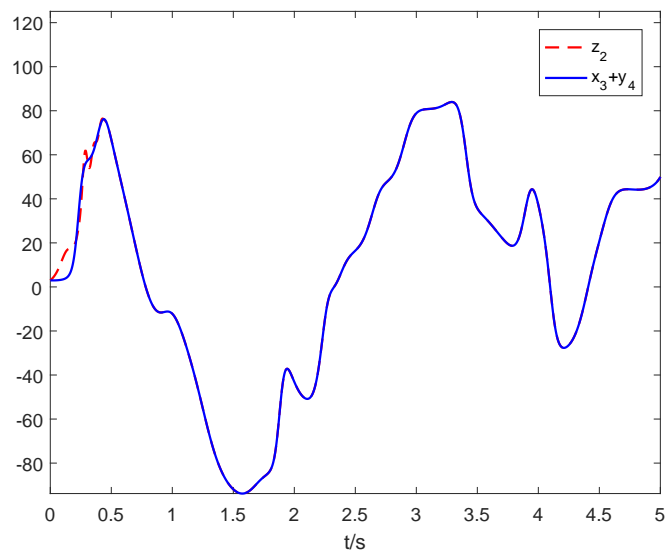

(b)

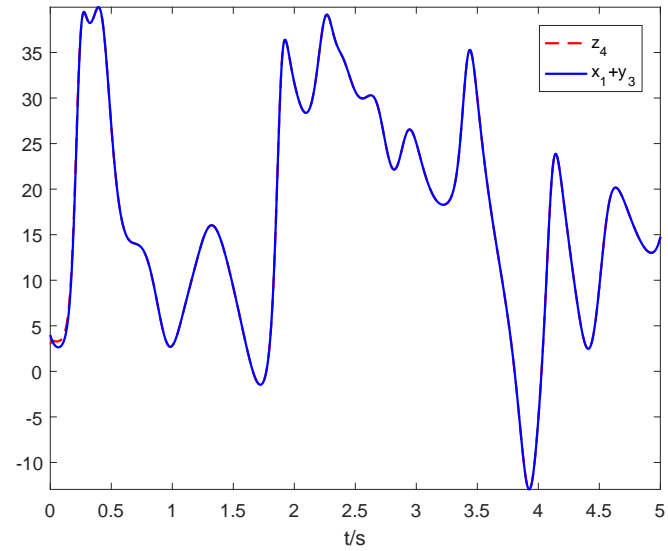

(d)

Fig.3. The synchronization for state variable $z_{1}$ and $x_{4}+y_{2}, z_{2}$ and $x_{3}+y_{4}, z_{3}$ and $x_{2}+y_{1}$, $z_{4}$ and $x_{1}+y_{3}$ of drive systems (34), (35) and response system (36) indicating in sub-pictures (a)-(d) respectively.

$(1,-1,3), z(0)=(-2,3,1,3)$. When $\Delta g=0, \Delta f=0, \Delta h=0, d(t)=0, D(t)=0$ ,$\mu(t)=0$ and $\alpha=0.97$, we can obtain the attractor graphs of the FO Lorenz, Chen chaotic system which are presented in figure 10. The attractor graphs of hyper-chaotic Lü system can be seen figure 1. Assume

$$
A=B=\left(\begin{array}{lll}
1 & 0 & 1 \\
2 & 1 & 0 \\
0 & 1 & 2 \\
1 & 0 & 1
\end{array}\right), \quad C=\left(\begin{array}{llll}
1 & 0 & 0 & 0 \\
0 & 1 & 0 & 0 \\
0 & 0 & 1 & 0 \\
0 & 0 & 0 & 1
\end{array}\right)
$$

The following multi-switching error state modes between the drive systems (40), (41) and the response system (42) are listed. 


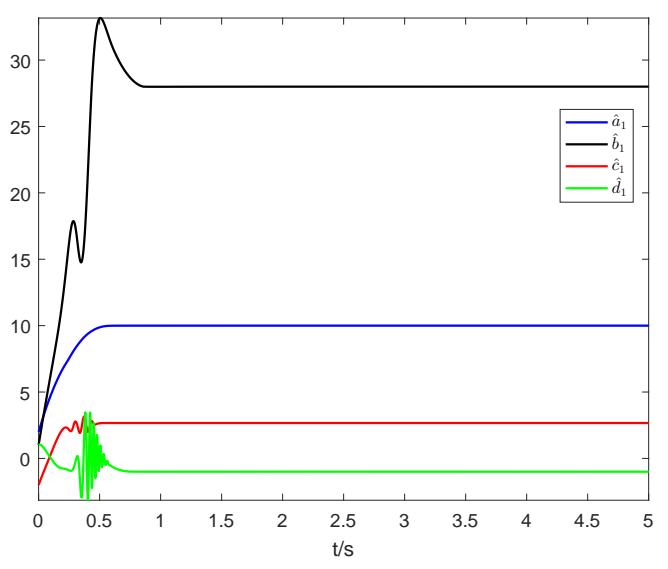

(a)

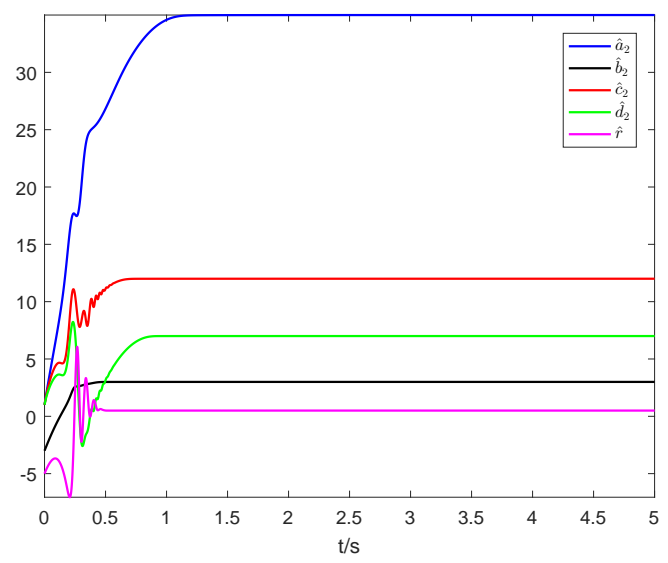

(b)

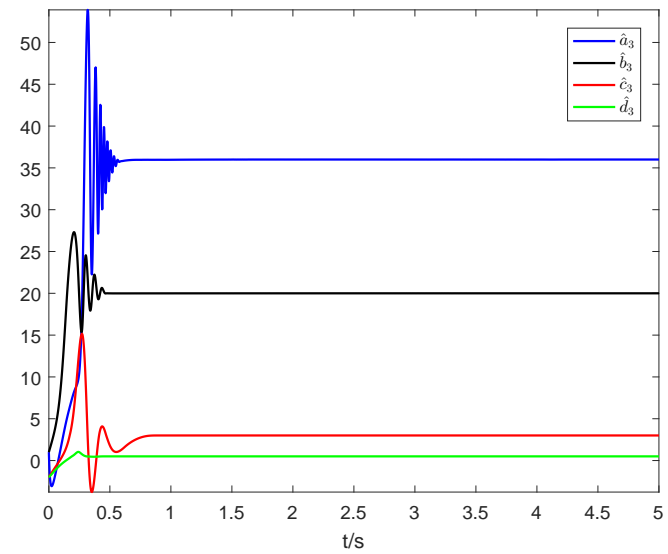

(c)

Fig.4. The estimation of parameters $\hat{a}_{1}, \hat{b}_{1}, \hat{c}_{1}, \hat{d}_{1}$ of drive system (34) (a), $\hat{a}_{2}, \hat{b}_{2}, \hat{c}_{2}, \hat{d}_{2}, \hat{r}$ of drive system (35) (b), $\hat{a}_{3}, \hat{b}_{3}, \hat{c}_{3}, \hat{d}_{3}$ of response system (36) (c).

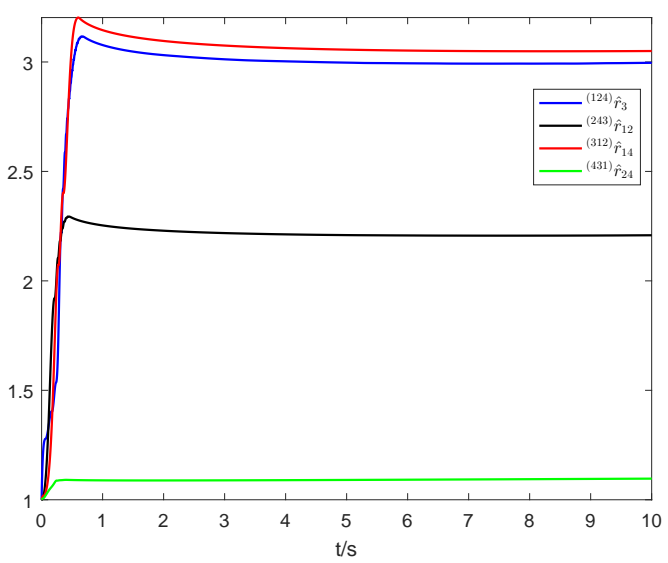

(a)

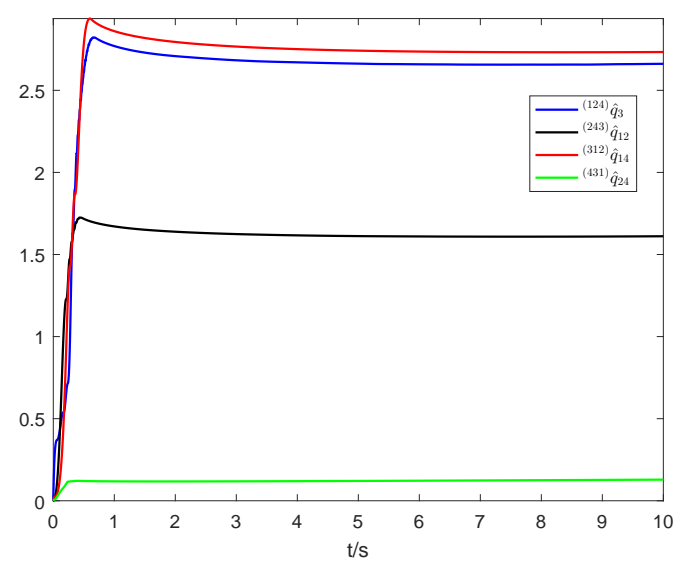

(b)

Fig.5. The estimation of parameters ${ }^{(124)} \hat{r}_{3},{ }^{(243)} \hat{r}_{12},{ }^{(312)} \hat{r}_{14},{ }^{(431)} \hat{r}_{24}$ and ${ }^{(124)} \hat{q}_{3},{ }^{(243)} \hat{q}_{12}$, ${ }^{(312)} \hat{q}_{14},{ }^{(431)} \hat{q}_{24}$ shown in sub-pictures (a) and (b). 


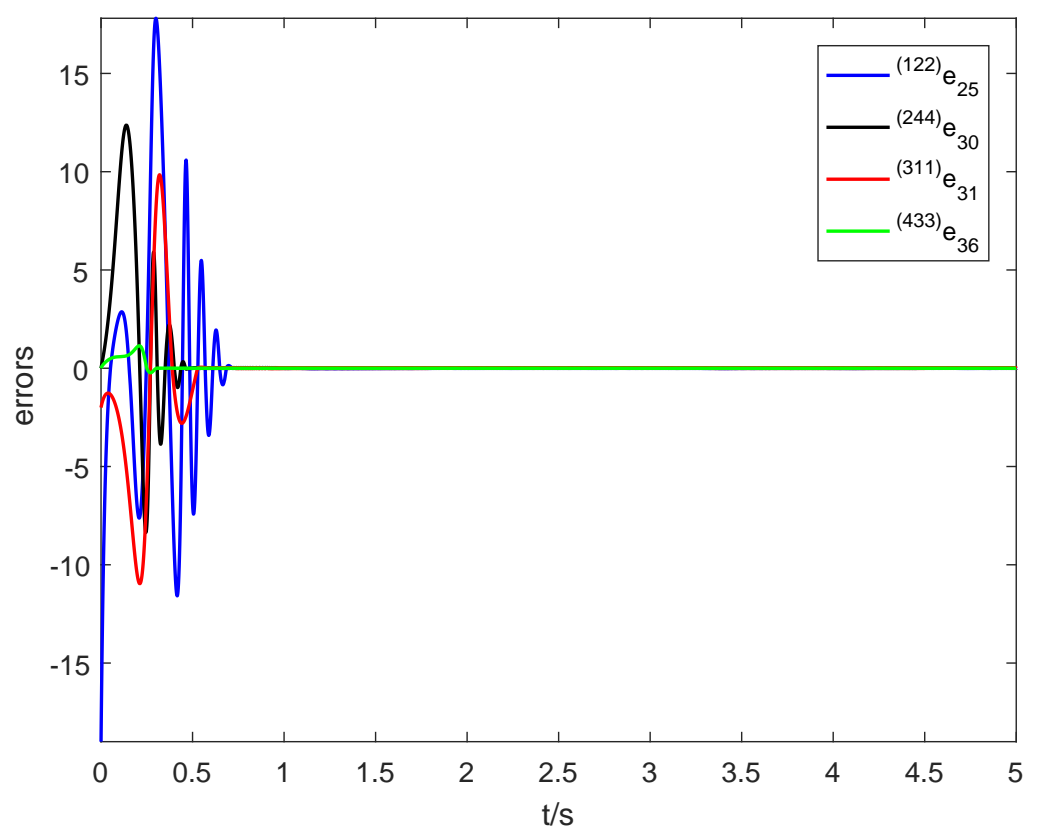

Fig.6. The synchronization errors ${ }^{(122)} e_{25},{ }^{(244)} e_{30},{ }^{(311)} e_{31},{ }^{(433)} e_{36}$ change with time $t$.

$$
\begin{aligned}
& l \neq w \neq v\left\{\begin{array}{llllll}
{ }^{(123)} e_{1}, & { }^{(132)} e_{2}, & { }^{(124)} e_{3}, & { }^{(142)} e_{4}, & { }^{(134)} e_{5}, & { }^{(143)} e_{6}, \\
{ }^{(213)} e_{7}, & { }^{(231)} e_{8}, & { }^{(214)} e_{9}, & { }^{(241)} e_{10}, & { }^{(234)} e_{11}, & { }^{(243)} e_{12} \\
{ }^{(321)} e_{13}, & { }^{(312)} e_{14}, & { }^{(324)} e_{15}, & { }^{(314)} e_{16}, & { }^{(341)} e_{17}, & { }^{(342)} e_{18} \\
{ }^{(412)} e_{19}, & { }^{(421)} e_{20}, & { }^{(413)} e_{21}, & { }^{(423)} e_{22}, & { }^{(432)} e_{23}, & { }^{(431)} e_{24}
\end{array}\right.
\end{aligned}
$$

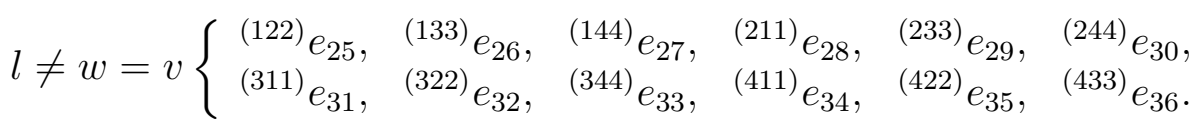

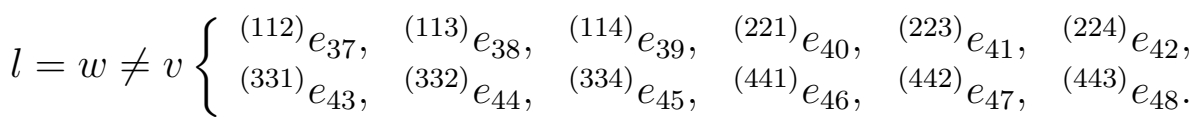

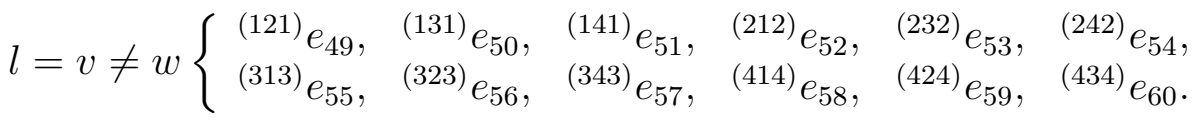

$$
\begin{aligned}
& l=w=v \begin{cases}{ }^{(111)} e_{61}, & { }^{(222)} e_{62}, \\
{ }^{(333)} e_{63}, & { }^{(444)} e_{64} .\end{cases}
\end{aligned}
$$

In this Section, we will select the appropriate switching error variable for the situation of $l \neq w \neq v$, namely, $\boldsymbol{e}=\left({ }^{(142)} e_{4},{ }^{(234)} e_{12},{ }^{\left({ }^{(321)}\right.} e_{13},{ }^{(413)} e_{21}\right)^{T}, l \neq w=v$, namely, $\boldsymbol{e}=\left({ }^{(122)} e_{25},{ }^{(244)} e_{30},{ }^{(311)} e_{31},{ }^{(433)} e_{36}\right)^{T}$ and obtain the following two switching modes for numerical simulation: 


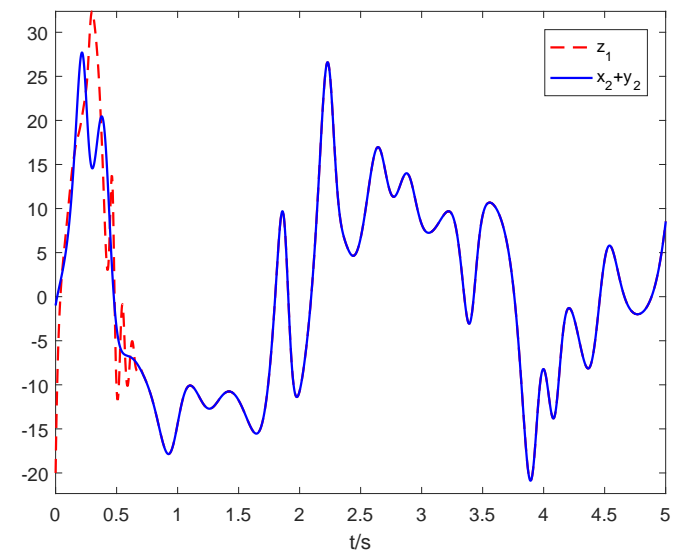

(a)

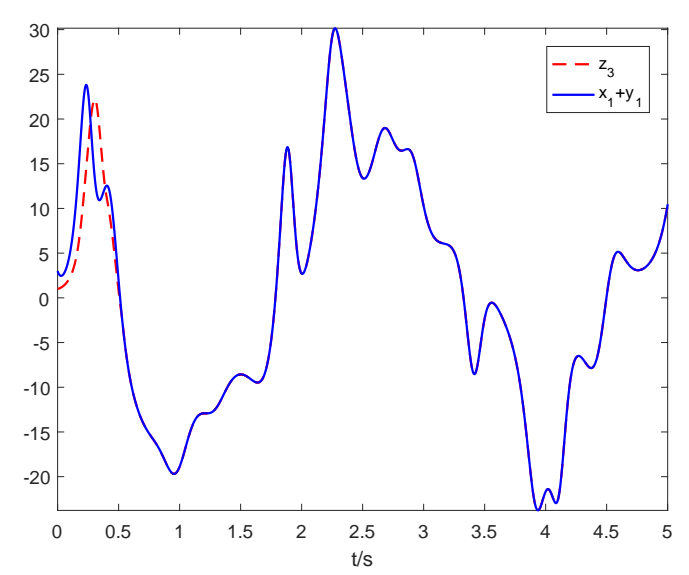

(c)

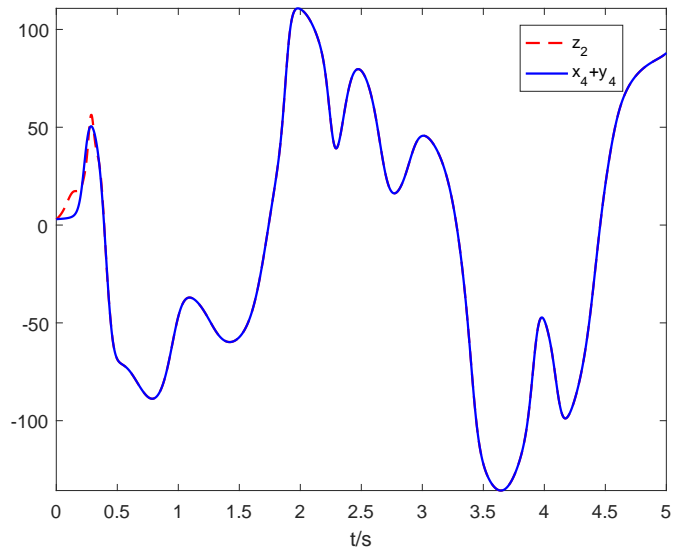

(b)

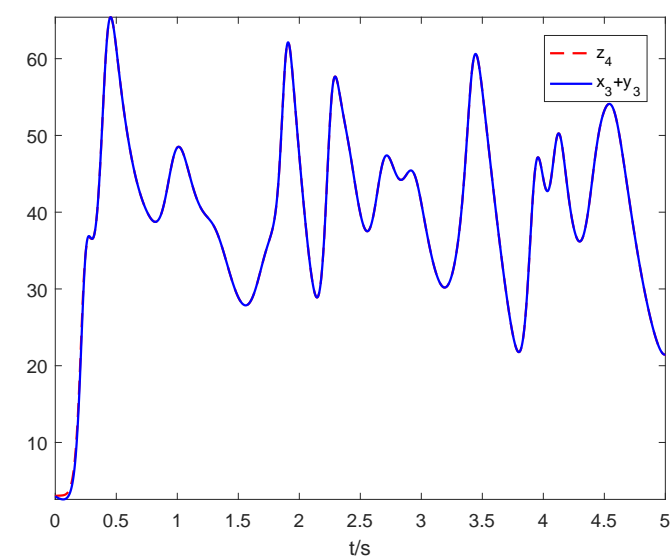

(d)

Fig.7. The synchronization for state variable $z_{1}$ and $x_{2}+y_{2}, z_{2}$ and $x_{4}+y_{4}, z_{3}$ and $x_{1}+y_{1}$, $z_{4}$ and $x_{3}+y_{3}$ of drive systems (34), (35) and response system (36) indicating in sub-pictures (a)-(d) respectively.

$$
\text { Switch-1: }\left\{\begin{aligned}
{ }^{(142)} e_{4}=\left[\sum_{i=1}^{4}\left(c_{1 i} z_{i}\right)-\left\{\sum_{j=1}^{3}\left(b_{4 j} y_{j}\right)+\sum_{k=1}^{3}\left(x_{2 k} x_{k}\right)\right\}\right], \\
{ }^{(234)} e_{12}=\left[\sum_{i=1}^{4}\left(c_{2 i} z_{i}\right)-\left\{\sum_{j=1}^{3}\left(b_{3 j} y_{j}\right)+\sum_{k=1}^{3}\left(x_{4 k} x_{k}\right)\right\}\right], \\
{ }^{(321)} e_{13}=\left[\sum_{i=1}^{4}\left(c_{3 i} z_{i}\right)-\left\{\sum_{j=1}^{3}\left(b_{2 j} y_{j}\right)+\sum_{k=1}^{3}\left(x_{1 k} x_{k}\right)\right\}\right], \\
{ }^{(413)} e_{21}=\left[\sum_{i=1}^{4}\left(c_{4 i} z_{i}\right)-\left\{\sum_{j=1}^{3}\left(b_{1 j} y_{j}\right)+\sum_{k=1}^{3}\left(x_{3 k} x_{k}\right)\right\}\right],
\end{aligned}\right.
$$




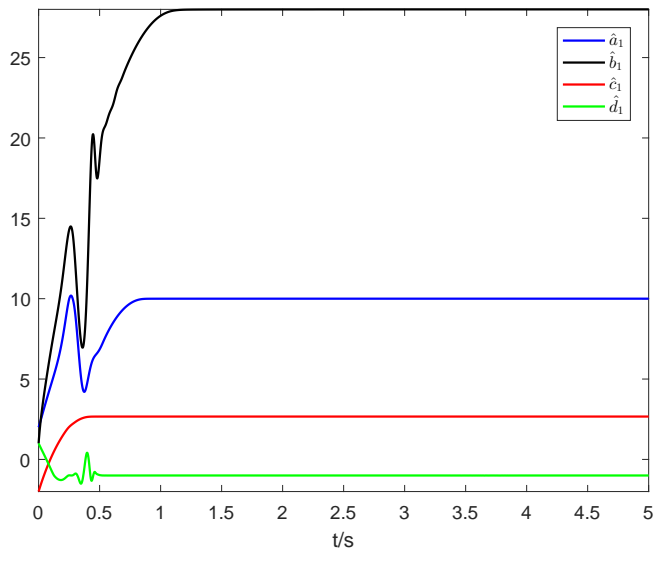

(a)

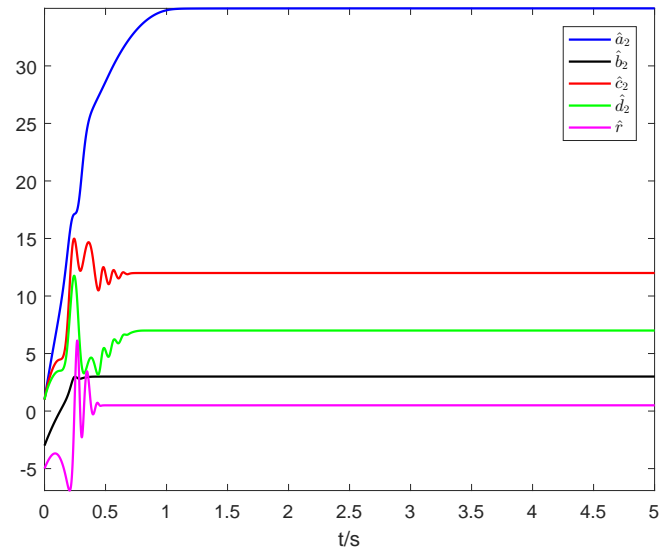

(b)

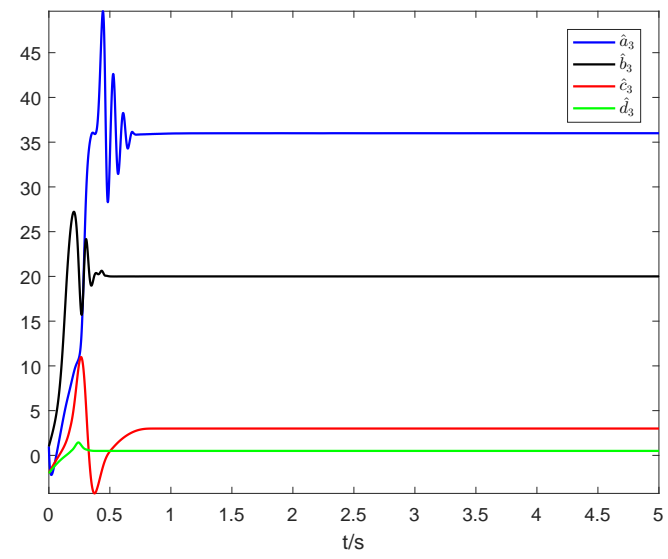

(c)

Fig.8. The estimation of parameters $\hat{a}_{1}, \hat{b}_{1}, \hat{c}_{1}, \hat{d}_{1}$ of drive system (34) (a), $\hat{a}_{2}, \hat{b}_{2}, \hat{c}_{2}, \hat{d}_{2}, \hat{r}$ of drive system (35) (b), $\hat{a}_{3}, \hat{b}_{3}, \hat{c}_{3}, \hat{d}_{3}$ of response system (36) (c).

$$
\text { Switch-2: }\left\{\begin{aligned}
{ }^{(122)} e_{25}=\left[\sum_{i=1}^{4}\left(c_{1 i} z_{i}\right)-\left\{\sum_{j=1}^{3}\left(b_{2 j} y_{j}\right)+\sum_{k=1}^{3}\left(x_{2 k} x_{k}\right)\right\}\right], \\
{ }^{(244)} e_{30}=\left[\sum_{i=1}^{4}\left(c_{2 i} z_{i}\right)-\left\{\sum_{j=1}^{3}\left(b_{4 j} y_{j}\right)+\sum_{k=1}^{3}\left(x_{4 k} x_{k}\right)\right\}\right], \\
{ }^{(311)} e_{31}=\left[\sum_{i=1}^{4}\left(c_{3 i} z_{i}\right)-\left\{\sum_{j=1}^{3}\left(b_{1 j} y_{j}\right)+\sum_{k=1}^{3}\left(x_{1 k} x_{k}\right)\right\}\right], \\
{ }^{(433)} e_{36}=\left[\sum_{i=1}^{4}\left(c_{4 i} z_{i}\right)-\left\{\sum_{j=1}^{3}\left(b_{3 j} y_{j}\right)+\sum_{k=1}^{3}\left(x_{3 k} x_{k}\right)\right\}\right],
\end{aligned}\right.
$$

In order to exhibit the effectiveness of the designed MSAC (25) and the MSAUL (28), 


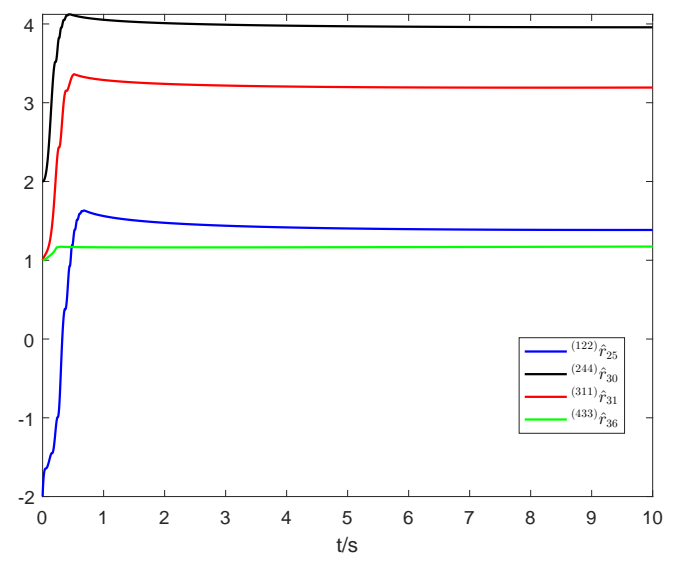

(a)

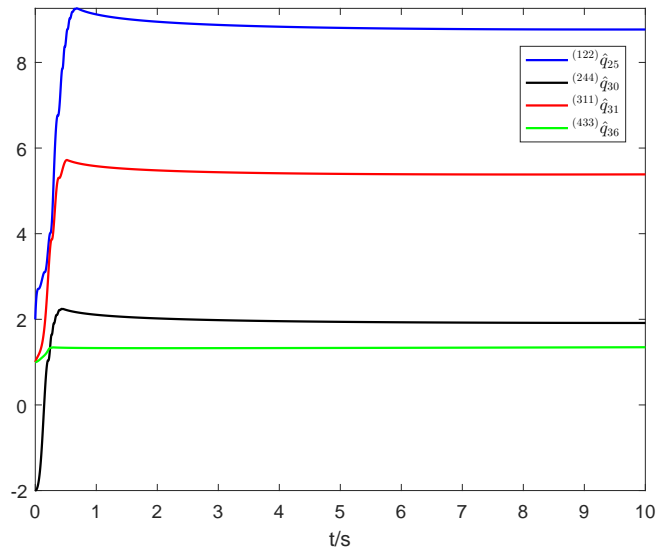

(b)

Fig.9. The estimation of parameters ${ }^{(122)} \hat{r}_{25},{ }^{(244)} \hat{r}_{30},{ }^{(311)} \hat{r}_{31},{ }^{(433)} \hat{r}_{36}$ and ${ }^{(122)} \hat{q}_{25},{ }^{(244)} \hat{q}_{30}$, ${ }^{(311)} \hat{q}_{31},{ }^{(433)} \hat{q}_{36}$ shown in sub-pictures (a) and (b).

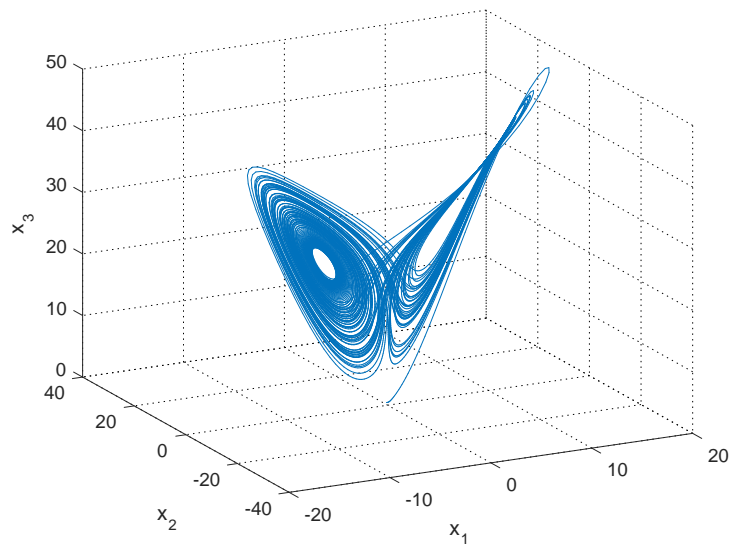

(a)

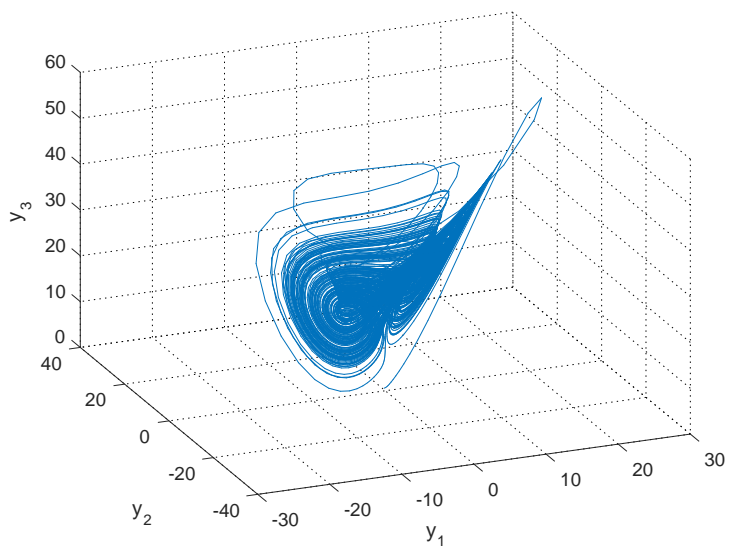

(b)

Fig.10. The 3D phase plot of FO Lorenz chaotic system (a), FO Chen chaotic system (b).

the nonlinear uncertainty and external disturbance are assumed as follows:

$$
\begin{aligned}
\boldsymbol{d}(\boldsymbol{x}(t)) & =(-0.1 \cos (t),-0.2 \cos (2 t), 0.3 \sin (3 t))^{T}, \\
\boldsymbol{D}(\boldsymbol{y}(t)) & =(-0.1 \sin (t),-0.2 \sin (2 t), 0.3 \cos (3 t))^{T}, \\
\boldsymbol{\mu}(\boldsymbol{z}(t)) & =(0.1 \cos (5 t), 0.2 \cos (6 t), 0.3 \sin (7 t), 0.4 \sin (8 t))^{T}, \\
\Delta \boldsymbol{f}(\boldsymbol{x}(t)) & =\left(0.1 \cos \left(2 t x_{1}\right), 0.2 \cos \left(2 t x_{2}\right), 0.3 \sin \left(2 t x_{3}\right)\right)^{T}, \\
\Delta \boldsymbol{g}(\boldsymbol{y}(t)) & =\left(0.1 \sin (2 \pi t), 0.2 \sin \left(\operatorname{sign}\left(y_{2}\right)\right), 0.3 \cos \left(2 \pi y_{3}\right)\right)^{T}, \\
\Delta \boldsymbol{h}(\boldsymbol{z}(t)) & =\left(0.1 \sin \left(2 t \operatorname{sign}\left(z_{1}\right)\right), 0.2 \sin \left(2 t \operatorname{sign}\left(z_{2}\right)\right), 0.3 \sin (3 t), 0.4 \sin (4 t)^{T} .\right.
\end{aligned}
$$




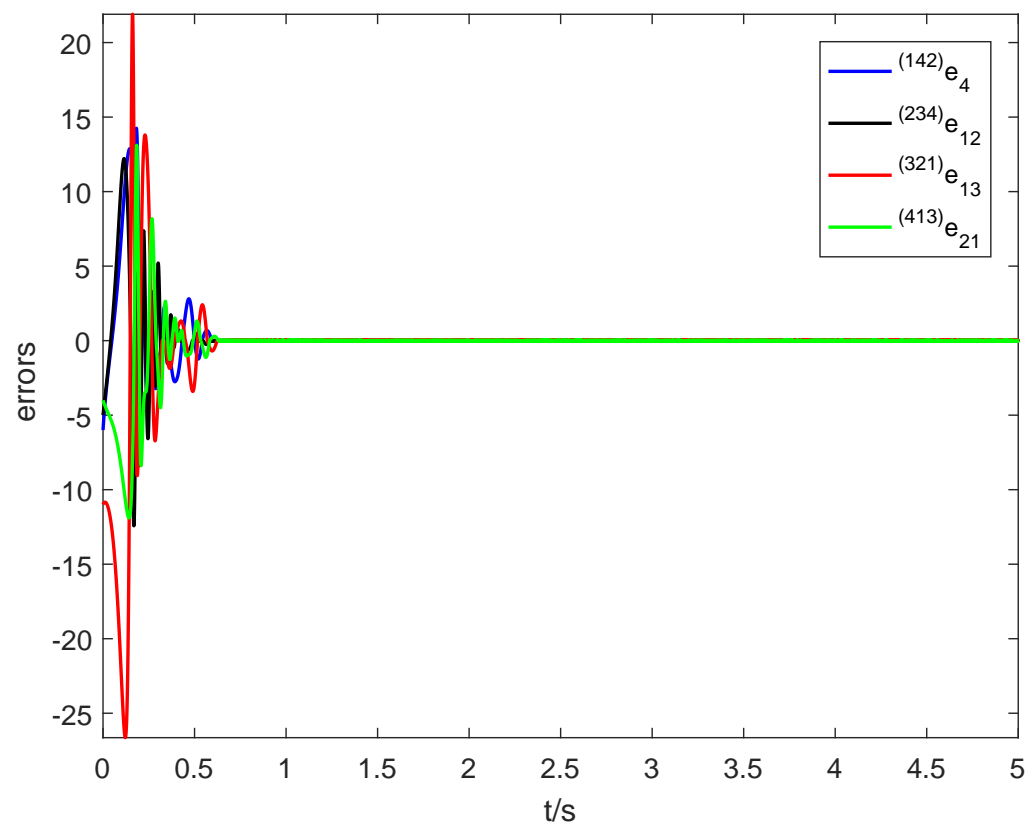

Fig.11. The synchronization errors ${ }^{(142)} e_{4},{ }^{(234)} e_{12},{ }^{(321)} e_{13},{ }^{(413)} e_{21}$ change with time $t$.

\subsubsection{Switch-1}

It follows from switch-1 (44) that the FO error dynamic system is expressed as:

$$
\begin{aligned}
{ }_{0} D_{t}^{\alpha}\left\{{ }^{(142)} e_{4}\right\}= & {\left[\sum_{i=1}^{4} c_{1 i}\left({ }_{0} D_{t}^{\alpha} z_{i}\right)-\left\{\sum_{j=1}^{3} b_{4 j}\left({ }_{0} D_{t}^{\alpha} y_{j}\right)+\sum_{k=1}^{3} x_{2 k}\left({ }_{0} D_{t}^{\alpha} x_{k}\right)\right\}\right] } \\
= & \left\{\left(z_{2}-z_{1}\right) a_{3}+z_{4}+\Delta h_{1}+\mu_{1}+{ }^{(142)} u_{4}\right\} \\
& -\left\{\left(y_{2}-y_{1}\right) a_{2}-y_{3} b_{2}+y_{1} y_{2}+\Delta g_{1}+\Delta g_{3}+D_{1}+D_{3}\right\} \\
& -\left\{2\left(x_{2}-x_{1}\right) a_{1}+x_{1} b_{1}-x_{1} x_{3}-x_{2}+2 \Delta f_{1}+\Delta f_{2}+2 d_{1}+d_{2}\right\}, \\
{ }_{0} D_{t}^{\alpha}\left\{{ }^{(234)} e_{12}\right\}= & {\left[\sum_{i=1} c_{2 i}\left({ }_{0} D_{t}^{\alpha} z_{i}\right)-\left\{\sum_{j=1}^{3} b_{3 j}\left({ }_{0} D_{t}^{\alpha} y_{j}\right)+\sum_{k=1}^{3} x_{4 k}\left({ }_{0} D_{t}^{\alpha} x_{k}\right)\right\}\right] } \\
= & \left\{z_{2} b_{3}-z_{1} z_{3}+\Delta h_{2}+\mu_{2}+{ }^{(234)} u_{12}\right\}-\left\{-y_{1} a_{2}-2 y_{3} b_{2}\right. \\
& \left.+\left(y_{2}+y_{1}\right) c_{2}-y_{1} y_{3}+2 y_{1} y_{2}+\Delta g_{2}+2 \Delta g_{3}+D_{2}+2 D_{3}\right\} \\
& -\left\{\left(x_{2}-x_{1}\right) a_{1}-x_{3} c_{1}+x_{1} x_{2}+\Delta f_{1}+\Delta f_{3}+d_{1}+d_{3}\right\}, \\
{ }_{0} D_{t}^{\alpha}\left\{{ }^{(321)} e_{13}\right\}= & {\left[\sum_{i=1}^{4} c_{3 i}\left({ }_{0} D_{t}^{\alpha} z_{i}\right)-\left\{\sum_{j=1}^{3} b_{2 j}\left({ }_{0} D_{t}^{\alpha} y_{j}\right)+\sum_{k=1}^{3} x_{1 k}\left({ }_{0} D_{t}^{\alpha} x_{k}\right)\right\}\right] } \\
= & \left\{-z_{3} c_{3}+z_{1} z_{2}+\Delta h_{3}+\mu_{3}+{ }^{(321)} u_{13}\right\}-\left\{2\left(y_{2}-2 y_{1}\right) a_{2}\right. \\
& \left.+\left(y_{1}+y_{2}\right) c_{2}-y_{1} a_{2}-y_{1} y_{3}+2 \Delta g_{1}+\Delta g_{2}+2 D_{1}+D_{2}\right\} \\
& -\left\{\left(x_{2}-x_{1}\right) a_{1}-x_{3} c_{1}-x_{1} x_{2}+\Delta f_{1}+\Delta f_{3}+d_{1}+d_{3}\right\}, \\
& 4 \\
& {\left[\sum_{i=1}^{3} c_{4 i}\left({ }_{0} D_{t}^{\alpha} z_{i}\right)-\left\{\sum_{j=1} b_{1 j}\left({ }_{0} D_{t}^{\alpha} y_{j}\right)+\sum_{k=1}^{3} x_{3 k}\left({ }_{0} D_{t}^{\alpha} x_{k}\right)\right\}\right] } \\
{ }_{0} D_{t}^{\alpha}\left\{{ }^{(413)} e_{21}\right\} &
\end{aligned}
$$




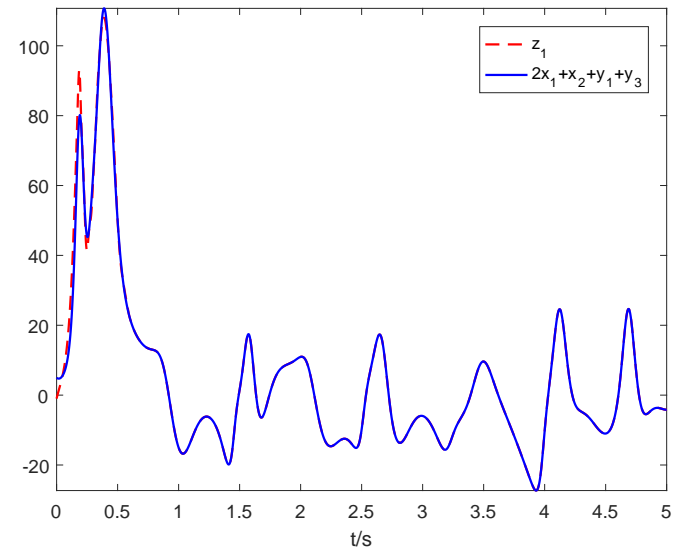

(a)

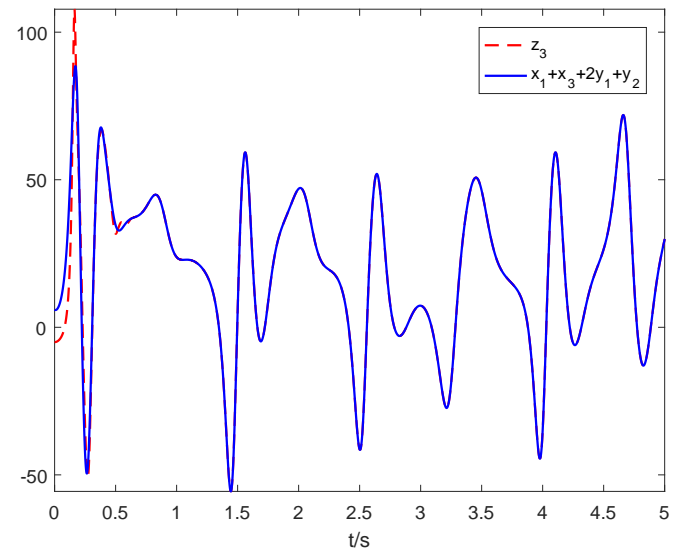

(c)

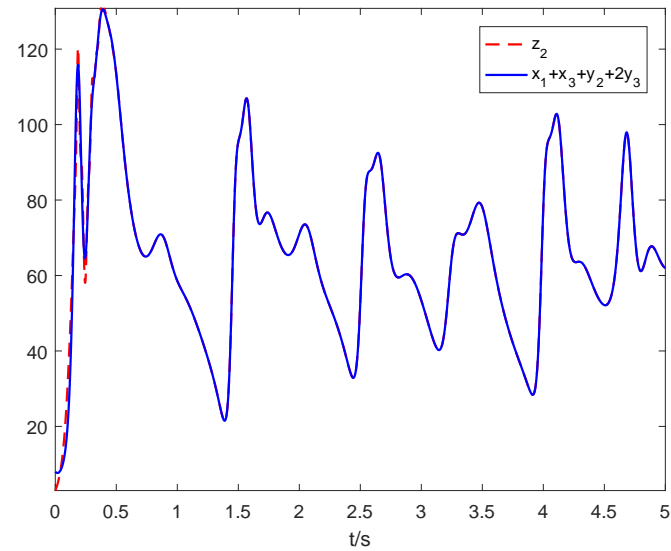

(b)

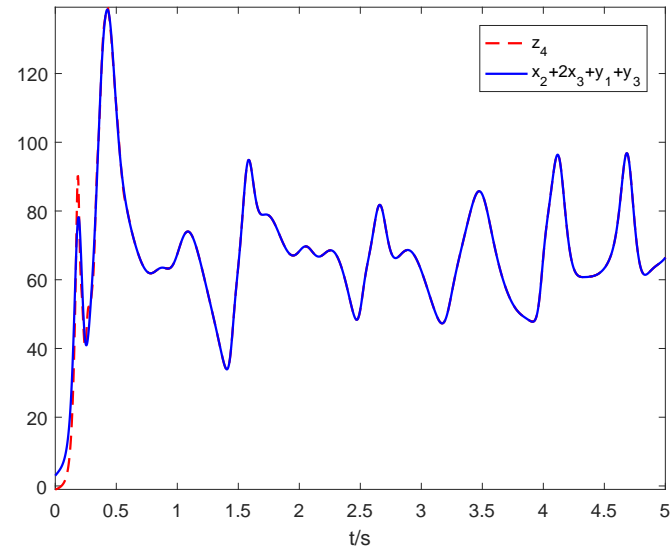

(d)

Fig.12. The synchronization for state variable $z_{1}$ and $2 x_{1}+x_{2}+y_{1}+y_{3}, z_{2}$ and $x_{1}+x_{3}+$ $y_{2}+2 y_{3}, z_{3}$ and $x_{1}+x_{3}+2 y_{1}+y_{2}, z_{4}$ and $x_{2}+2 x_{3}+y_{1}+y_{3}$ of drive systems (40), (41) and response system (42) indicating in sub-pictures (a)-(d) respectively.

$$
\begin{aligned}
& =\left\{z_{4} d_{3}+z_{1} z_{3}+\Delta h_{4}+\mu_{4}+{ }^{(413)} u_{21}\right\} \\
& -\left\{\left(y_{2}-y_{1}\right) a_{2}-y_{3} b_{2}+y_{1} y_{2}+\Delta g_{1}+\Delta g_{3}+D_{1}+D_{3}\right\} \\
& -\left\{x_{1} b_{1}-2 x_{3} c_{1}-x_{1} x_{3}-x_{2}+2 x_{1} x_{2}+\Delta f_{2}+2 \Delta f_{3}+d_{2}+2 d_{3}\right\} .
\end{aligned}
$$

It follows from the form of MSAC (25) and MSAUL (28) that the controller is designed as follows:

$$
\begin{aligned}
{ }^{(142)} u_{4} & =-z_{4}+y_{1} y_{2}-x_{1} x_{3}-x_{2}-\hat{a}_{3}\left(z_{2}-z_{1}\right)+\hat{a}_{2}\left(y_{2}-y_{1}\right)-\hat{b}_{1} y_{3} \\
& +2 \hat{a}_{1}\left(x_{2}-x_{1}\right)+\hat{b}_{1} x_{1}-\left({ }^{(142)} \hat{\rho}_{4}+{ }^{(142)} \hat{\varrho}_{4}\right) \operatorname{sign}\left({ }^{(142)} s_{4}\right)-k_{1} \tanh \left({ }^{(142)} s_{4}\right), \\
{ }^{(234)} u_{12} & =z_{1} z_{3}-y_{1} y_{3}+2 y_{1} y_{2}+x_{1} x_{2}-\hat{b}_{3} z_{2}-\hat{a}_{2} y_{1}-2 \hat{b}_{2} y_{3}+\hat{c}_{2}\left(y_{1}+y_{2}\right) \\
& +\hat{a}_{1}\left(x_{2}-x_{1}\right)-\hat{c}_{1} x_{3}-\left({ }^{(234)} \hat{\rho}_{12}+{ }^{(234)} \hat{\varrho}_{12}\right) \operatorname{sign}\left({ }^{(234)} s_{12}\right)-k_{1} \tanh \left({ }^{(234)} s_{12}\right), \\
{ }^{(321)} u_{13} & =-z_{1} z_{2}-y_{1} y_{3}+x_{1} x_{2}+\hat{c}_{3} z_{3}-\hat{a}_{2} y_{3}+2 \hat{a}_{2}\left(y_{2}-2 y_{1}\right)+\hat{c}_{2}\left(y_{1}+y_{2}\right) \\
& +\hat{a}_{1}\left(x_{2}-x_{1}\right)-\hat{c}_{1} x_{3}-\left({ }^{(321)} \hat{\rho}_{13}+{ }^{(321)} \hat{\varrho}_{13}\right) \operatorname{sign}\left({ }^{(321)} s_{13}\right)-k_{1} \tanh \left({ }^{(321)} s_{13}\right), \\
{ }^{(413)} u_{21} & =-z_{1} z_{3}+y_{1} y_{2}-x_{1} x_{3}-x_{2}+2 x_{1} x_{2}-d_{3} z_{4}+a_{2}\left(y_{2}-y_{1}\right)-b_{2} y_{3}
\end{aligned}
$$




$$
+b_{1} x_{1}-2 c_{1} x_{3}-\left({ }^{(413)} \hat{\rho}_{21}+{ }^{(413)} \hat{\varrho}_{21}\right) \operatorname{sign}\left({ }^{(413)} s_{21}\right)-k_{1} \tanh \left({ }^{(413)} s_{21}\right) \text {, }
$$

and the parameters updating laws are designed as follows:

$$
\begin{aligned}
& \hat{b}_{1}=-x_{1}\left\{\left({ }^{(142)} s_{4}\right)+\left({ }^{(413)} s_{21}\right)\right\}-k_{2} \operatorname{sign}\left(\tilde{b}_{1}\right)\left(\operatorname{abs}\left(\tilde{b}_{1}\right)^{\omega}\right), \\
& \hat{c}_{1}=x_{3}\left\{\left({ }^{(234)} s_{12}\right)+\left({ }^{(321)} s_{13}\right)+2\left({ }^{(142)} s_{4}\right)\right\}-k_{2} \operatorname{sign}\left(\tilde{c}_{1}\right)\left(\operatorname{abs}\left(\tilde{c}_{1}\right)^{\omega}\right) \text {, } \\
& \hat{b}_{2}=y_{3}\left\{\left({ }^{(142)} s_{4}\right)+2\left({ }^{(234)} s_{12}\right)+\left({ }^{(413)} s_{21}\right)\right\}-k_{3} \operatorname{sign}\left(\tilde{b}_{2}\right)\left(\operatorname{abs}\left(\tilde{b}_{2}\right)^{\omega}\right) \text {, } \\
& \hat{c}_{2}=-\left(y_{1}+y_{2}\right)\left\{\left({ }^{(234)} s_{12}\right)+\left({ }^{(321)} s_{13}\right)\right\}-k_{3} \operatorname{sign}\left(\tilde{c}_{2}\right)\left(\operatorname{abs}\left(\tilde{c}_{2}\right)^{\omega}\right) \text {, } \\
& \hat{a}_{3}=\left(z_{2}-z_{1}\right)\left({ }^{(142)} s_{4}\right)-k_{4} \operatorname{sign}\left(\tilde{a}_{3}\right)\left(\operatorname{abs}\left(\tilde{a}_{3}\right)^{\omega}\right) \text {, } \\
& \hat{b}_{3}=z_{2}\left({ }^{(234)} s_{12}\right)-k_{4} \operatorname{sign}\left(\tilde{b}_{3}\right)\left(\operatorname{abs}\left(\tilde{b}_{3}\right)^{\omega}\right) \text {, } \\
& \hat{c}_{3}=-z_{3}\left({ }^{(321)} s_{13}\right)-k_{4} \operatorname{sign}\left(\tilde{c}_{3}\right)\left(\operatorname{abs}\left(\tilde{c}_{3}\right)^{\omega}\right) \text {, } \\
& \hat{d}_{3}=z_{4}\left({ }^{(413)} s_{21}\right)-k_{4} \operatorname{sign}\left(\tilde{d}_{3}\right)\left(\operatorname{abs}\left(\tilde{d}_{3}\right)^{\omega}\right) \text {, } \\
& \hat{a}_{1}=-\left(x_{2}-x_{1}\right)\left\{\left({ }^{(321)} s_{13}\right)+\left({ }^{(234)} s_{12}\right)+2\left({ }^{(413)} s_{21}\right)\right\}-k_{2} \operatorname{sign}\left(\tilde{a}_{1}\right)\left(\operatorname{abs}\left(\tilde{a}_{1}\right)^{\omega}\right) \text {, } \\
& \hat{a}_{2}=-\left\{\left(y_{2}-y_{1}\right)\left(\left({ }^{(142)} s_{4}\right)+\left({ }^{(413)} s_{21}\right)+2\left({ }^{(321)} s_{13}\right)\right)-y_{3}\left(\left({ }^{(234)} s_{12}\right)+\left({ }^{(321)} s_{13}\right)\right)\right\} \\
& -k_{1} \operatorname{sign}\left(\tilde{a}_{2}\right)\left(\operatorname{abs}\left(\tilde{a}_{2}\right)^{\omega}\right) \text {, } \\
& \begin{array}{ll}
{ }^{(142)} \hat{\rho}_{4}=\left.m_{1}\left({ }^{(142)} \lambda_{4}\right)\right|^{(142)} s_{4} \mid, & { }^{(234)} \hat{\rho}_{12}=\left.m_{1}\left\{{ }^{(234)} \lambda_{12}\right\}\right|^{(234)} s_{12} \mid, \\
{ }^{(321)} \hat{\rho}_{13}=\left.m_{1}\left\{{ }^{(321)} \lambda_{13}\right\}\right|^{(321)} s_{13} \mid, & { }^{(413)} \hat{\rho}_{21}=\left.m_{1}\left\{{ }^{(413)} \lambda_{21}\right\}\right|^{(413)} s_{21} \mid, \\
{ }^{(142)} \hat{\varrho}_{4}=\left.m_{2}\left({ }^{(142)} \lambda_{4}\right)\right|^{(142)} s_{4} \mid, & { }^{(234)} \hat{\varrho}_{12}=\left.m_{2}\left({ }^{(234)} \lambda_{12}\right)\right|^{(234)} s_{12} \mid, \\
{ }^{(321)} \hat{\varrho}_{13}=\left.m_{2}\left({ }^{(321)} \lambda_{13}\right)\right|^{(321)} s_{13} \mid, & { }^{(413)} \hat{\varrho}_{21}=\left.m_{2}\left({ }^{(413)} \lambda_{21}\right)\right|^{(413)} s_{21} \mid .
\end{array}
\end{aligned}
$$

\subsubsection{Switch-2}

It follows from switch-2 (45) that the FO error dynamic system is expressed as:

$$
\begin{aligned}
{ }_{0} D_{t}^{\alpha}\left\{{ }^{(122)} e_{25}\right\}= & {\left[\sum_{i=1}^{4} c_{1 i}\left({ }_{0} D_{t}^{\alpha} z_{i}\right)-\left\{\sum_{j=1}^{3} b_{2 j}\left({ }_{0} D_{t}^{\alpha} y_{j}\right)+\sum_{k=1}^{3} x_{2 k}\left({ }_{0} D_{t}^{\alpha} x_{k}\right)\right\}\right] } \\
= & \left\{\left(z_{2}-z_{1}\right) a_{3}+z_{4}+\Delta h_{1}+\mu_{1}+{ }^{(122)} u_{25}\right\}-\left\{2\left(y_{2}-y_{1}\right) a_{2}\right. \\
& \left.-y_{1} a_{2}+\left(y_{1}+y_{2}\right) c_{2}-y_{1} y_{3}+2 \Delta g_{1}+\Delta g_{2}+2 D_{1}+D_{2}\right\} \\
& -\left\{2\left(x_{2}-x_{1}\right) a_{1}+x_{1} b_{1}-x_{1} x_{3}-x_{2}+2 \Delta f_{1}+\Delta f_{2}+2 d_{1}+d_{2}\right\}, \\
{ }_{0} D_{t}^{\alpha}\left\{{ }^{(244)} e_{30}\right\}= & {\left[\sum_{i=1} c_{2 i}\left({ }_{0} D_{t}^{\alpha} z_{i}\right)-\left\{\sum_{j=1}^{3} b_{4 j}\left({ }_{0} D_{t}^{\alpha} y_{j}\right)+\sum_{k=1}^{3} x_{4 k}\left({ }_{0} D_{t}^{\alpha} x_{k}\right)\right\}\right] } \\
= & \left\{z_{2} b_{3}-z_{1} z_{3}+\Delta h_{2}+\mu_{2}+{ }^{(244)} u_{30}\right\} \\
& -\left\{\left(y_{2}-y_{1}\right) a_{2}-y_{3} b_{2}+y_{1} y_{2}+\Delta g_{1}+\Delta g_{3}+D_{1}+D_{3}\right\} \\
& -\left\{\left(x_{2}-x_{1}\right) a_{1}-x_{3} c_{1}+x_{1} x_{2}+\Delta f_{1}+\Delta f_{3}+d_{1}+d_{3}\right\}, \\
{ }_{0} D_{t}^{\alpha}\left\{{ }^{(311)} e_{31}\right\}= & {\left[\sum_{i=1} c_{3 i}\left({ }_{0}\left(D_{t}^{\alpha} z_{i}\right)-\left\{\sum_{j=1} b_{1 j}\left({ }_{0} D_{t}^{\alpha} y_{j}\right)+\sum_{k=1}^{3} x_{1 k 0} D_{t}^{\alpha} x_{k}\right)\right\}\right] } \\
= & \left\{-z_{3} c_{3}+z_{1} z_{2}+\Delta h_{3}+\mu_{3}+{ }^{(311)} u_{31}\right\} \\
& -\left\{\left(y_{2}-y_{1}\right) a_{2}-y_{3} b_{2}+y_{1} y_{2}+\Delta g_{1}+\Delta g_{3}+D_{1}+D_{3}\right\} \\
& -\left\{\left(x_{2}-x_{1}\right) a_{1}-x_{3} c_{1}+x_{1} x_{2}+\Delta f_{1}+\Delta f_{3}+d_{1}+d_{3}\right\},
\end{aligned}
$$




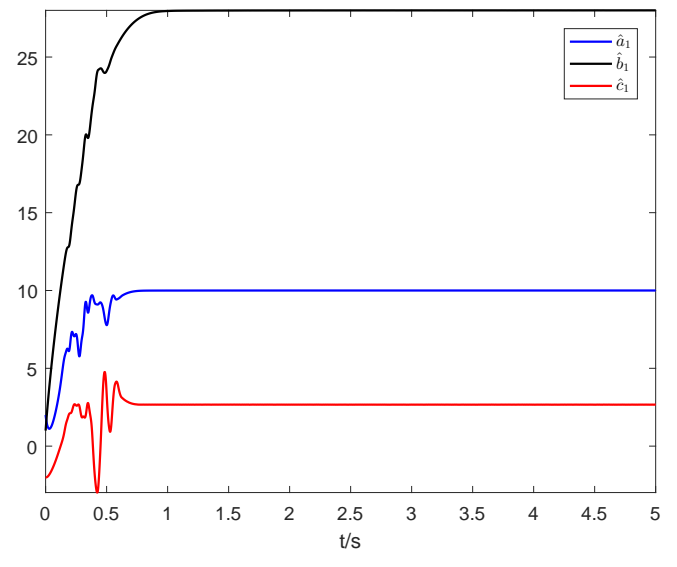

(a)

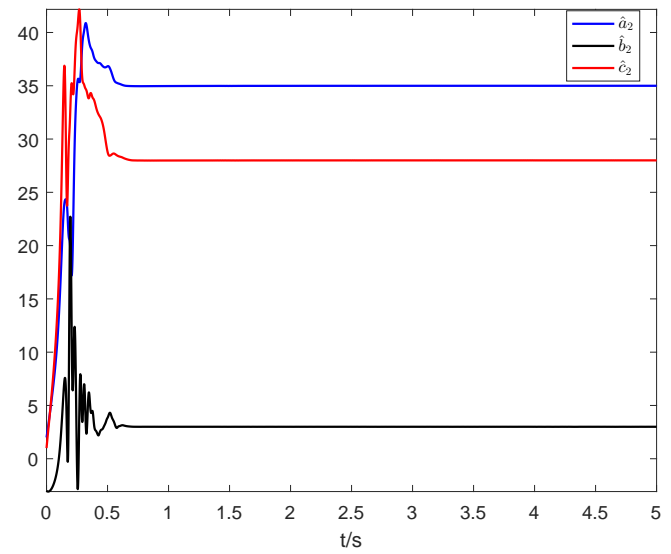

(b)

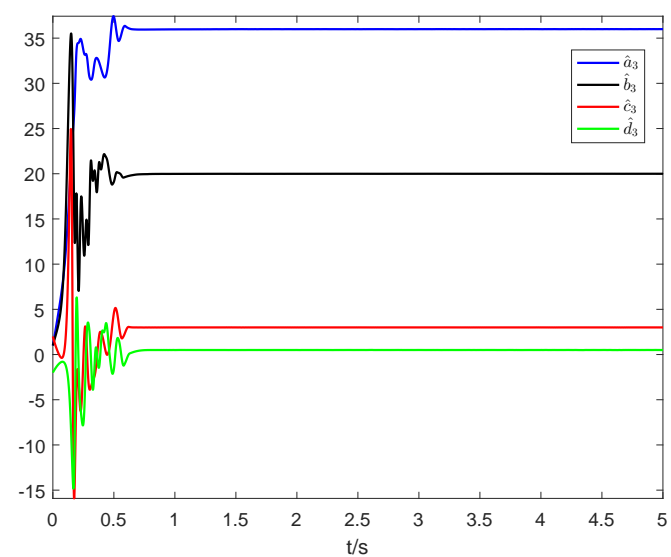

(c)

Fig.13. The estimation of parameters $\hat{a}_{1}, \hat{b}_{1}, \hat{c}_{1}$ of drive system (40) (a), $\hat{a}_{2}, \hat{b}_{2}, \hat{c}_{2}$ of drive system (41) (b), $\hat{a}_{3}, \hat{b}_{3}, \hat{c}_{3}, \hat{d}_{3}$ of drive system (42) (c).

$$
\begin{aligned}
{ }_{0} D_{t}^{\alpha}\left\{{ }^{(433)} e_{36}\right\} & =\left[\sum_{i=1}^{4} c_{4 i}\left({ }_{0} D_{t}^{\alpha} z_{i}\right)-\left\{\sum_{j=1}^{3} b_{3 j}\left({ }_{0} D_{t}^{\alpha} y_{j}\right)+\sum_{k=1}^{3} x_{3 k}\left({ }_{0} D_{t}^{\alpha} x_{k}\right)\right\}\right] \\
& =\left\{z_{4} d_{3}+z_{1} z_{3}+\Delta h_{4}+\mu_{4}+{ }^{(433)} u_{36}\right\}-\left\{-y_{1} a_{2}-2 y_{3} b_{2}\right. \\
& \left.+\left(y_{2}+y_{1}\right) c_{2}-y_{1} y_{3}+2 y_{1} y_{2}+\Delta g_{2}+2 \Delta g_{3}+D_{2}+2 D_{3}\right\} \\
& -\left\{x_{1} b_{1}-2 x_{3} c_{1}-x_{1} x_{3}-x_{2}+2 x_{1} x_{2}+\Delta f_{2}+2 \Delta f_{3}+d_{2}+2 d_{3}\right\} .
\end{aligned}
$$

It follows from the form of MSAC (25) and MSAUL (28) that the controller is designed as follows:

$$
\begin{aligned}
{ }^{(122)} u_{25} & =-z_{4}-y_{1} y_{3}-x_{1} x_{3}-x_{2}-\hat{a}_{3}\left(z_{2}-z_{1}\right)+2 \hat{a}_{2}\left(y_{2}-2 y_{1}\right)+\hat{c}_{2}\left(y_{1}+y_{2}\right)-\hat{a}_{2} y_{1} \\
& +2 \hat{a}_{1}\left(x_{2}-x_{1}\right)+\hat{b}_{1} x_{1}-\left({ }^{(122)} \hat{\rho}_{25}+{ }^{(122)} \hat{\varrho}_{25}\right) \operatorname{sign}\left({ }^{(122)} s_{25}\right)-k_{1} \tanh \left({ }^{(122)} s_{25}\right), \\
{ }^{(244)} u_{30} & =z_{1} z_{3}+y_{1} y_{2}+x_{1} x_{2}-\hat{b}_{3} z_{2}+\hat{a}_{2}\left(y_{2}-y_{1}\right)-\hat{b}_{1} y_{3} \\
& +\hat{a}_{1}\left(x_{2}-x_{1}\right)-\hat{c}_{1} x_{3}-\left({ }^{(244)} \hat{\rho}_{30}+{ }^{(244)} \hat{\varrho}_{30}\right) \operatorname{sign}\left({ }^{(244)} s_{30}\right)-k_{1} \tanh \left({ }^{(244)} s_{30}\right), \\
{ }^{(311)} u_{31} & =-z_{1} z_{2}+y_{1} y_{2}+x_{1} x_{2}+\hat{c}_{3} z_{3}+a_{2}\left(y_{2}-y_{1}\right)-b_{2} y_{3}
\end{aligned}
$$




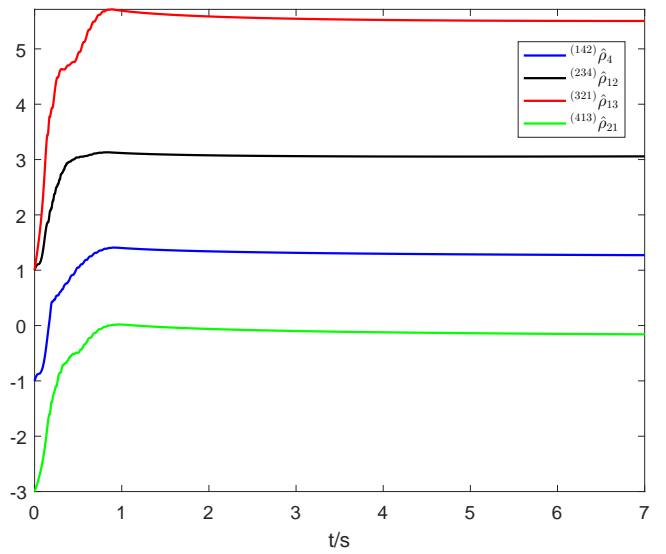

(a)

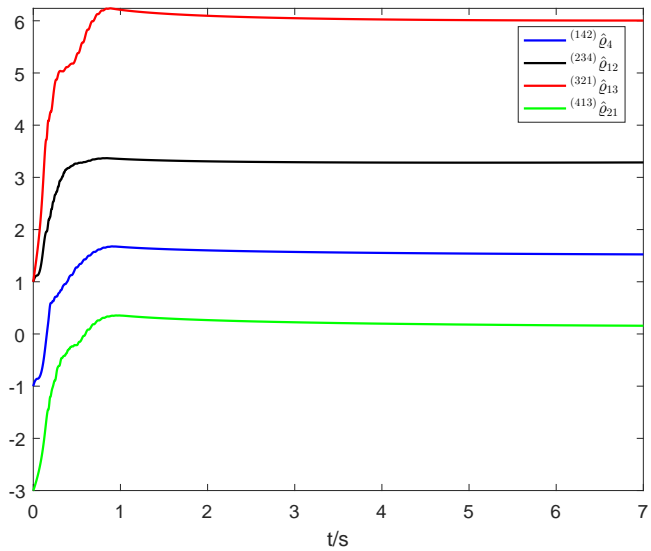

(b)

Fig.14. The estimation of parameters ${ }^{(142)} \hat{\rho}_{4},{ }^{(234)} \hat{\rho}_{12},{ }^{(321)} \hat{\rho}_{13},{ }^{(413)} \hat{\rho}_{21}$ and ${ }^{(142)} \hat{\varrho}_{4},{ }^{(234)} \hat{\varrho}_{12}$, ${ }^{(321)} \hat{\varrho}_{13},{ }^{(413)} \hat{\varrho}_{21}$ shown in sub-pictures (a) and (b).

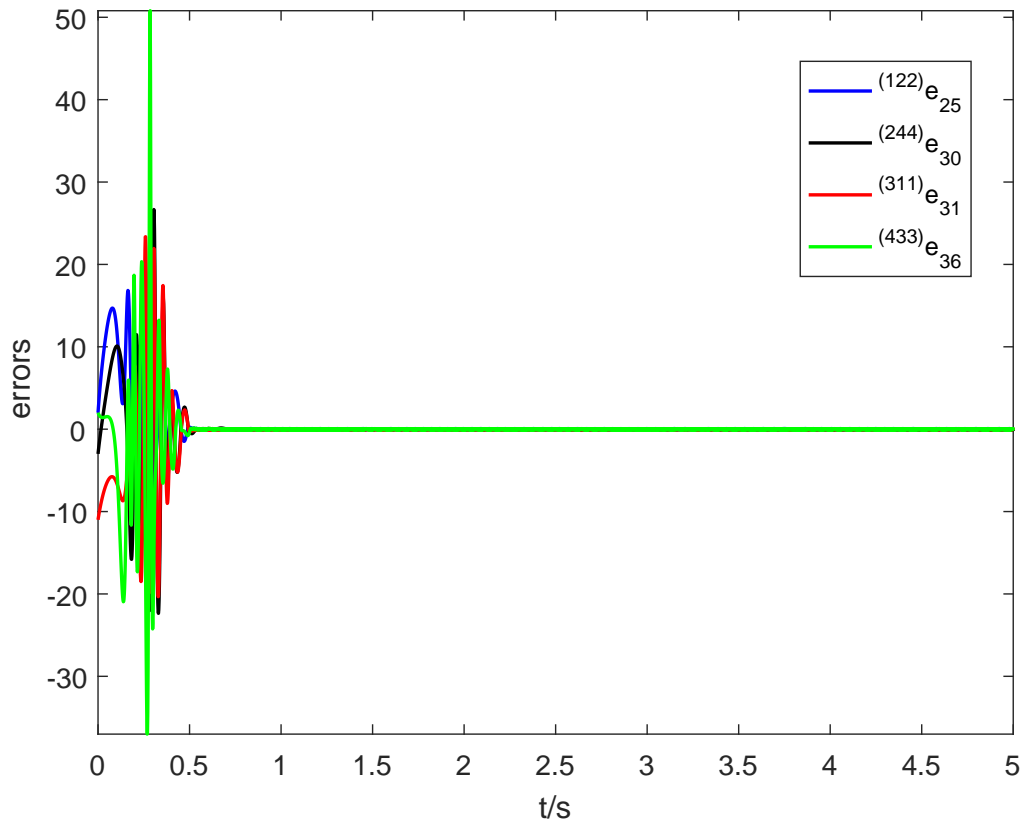

Fig.15. The synchronization errors ${ }^{(122)} e_{25},{ }^{(244)} e_{30},{ }^{(311)} e_{31},{ }^{(433)} e_{36}$ change with time $t$.

$$
\begin{aligned}
& +\hat{a}_{1}\left(x_{2}-x_{1}\right)-\hat{c}_{1} x_{3}-\left({ }^{(311)} \hat{\rho}_{31}+{ }^{(311)} \hat{\varrho}_{31}\right) \operatorname{sign}\left({ }^{(311)} s_{31}\right)-k_{1} \tanh \left({ }^{(311)} s_{31}\right), \\
{ }^{(433)} u_{36} & =-z_{1} z_{3}-y_{1} y_{3}+2 y_{1} y_{2}-x_{1} x_{3}-x_{2}+2 x_{1} x_{2}-d_{3} z_{4}-\hat{a}_{2} y_{1}-2 \hat{b}_{2} y_{3}+\hat{c}_{2}\left(y_{1}+y_{2}\right) \\
& +b_{1} x_{1}-2 c_{1} x_{3}-\left({ }^{(433)} \hat{\rho}_{36}+{ }^{(433)} \hat{\varrho}_{36}\right) \operatorname{sign}\left({ }^{(433)} s_{36}\right)-k_{1} \tanh \left({ }^{(433)} s_{36}\right) .
\end{aligned}
$$

and the parameters updating laws are designed as follows:

$$
\begin{array}{ll}
{ }^{(122)} \hat{\rho}_{25}=\left.m_{1}\left({ }^{(122)} \lambda_{25}\right)\right|^{(122)} s_{25} \mid, & { }^{(244)} \hat{\rho}_{30}=\left.m_{1}\left({ }^{(244)} \lambda_{30}\right)\right|^{(244)} s_{30} \mid, \\
{ }^{(311)} \hat{\rho}_{31}=\left.m_{1}\left({ }^{(311)} \lambda_{31}\right)\right|^{(311)} s_{31} \mid, & { }^{(433)} \hat{\rho}_{36}=\left.m_{1}\left({ }^{(433)} \lambda_{36}\right)\right|^{(433)} s_{36} \mid, \\
{ }^{(122)} \hat{\varrho}_{25}=\left.m_{2}\left({ }^{(122)} \lambda_{25}\right)\right|^{(122)} s_{25} \mid, & { }^{(244)} \hat{\varrho}_{30}=\left.m_{2}\left({ }^{(244)} \lambda_{30}\right)\right|^{(244)} s_{30} \mid, \\
{ }^{(311)} \hat{\varrho}_{31}=\left.m_{2}\left({ }^{(311)} \lambda_{31}\right)\right|^{(311)} s_{31} \mid, & { }^{(433)} \hat{\varrho}_{36}=\left.m_{2}\left({ }^{(433)} \lambda_{36}\right)\right|^{(433)} s_{36} \mid,
\end{array}
$$




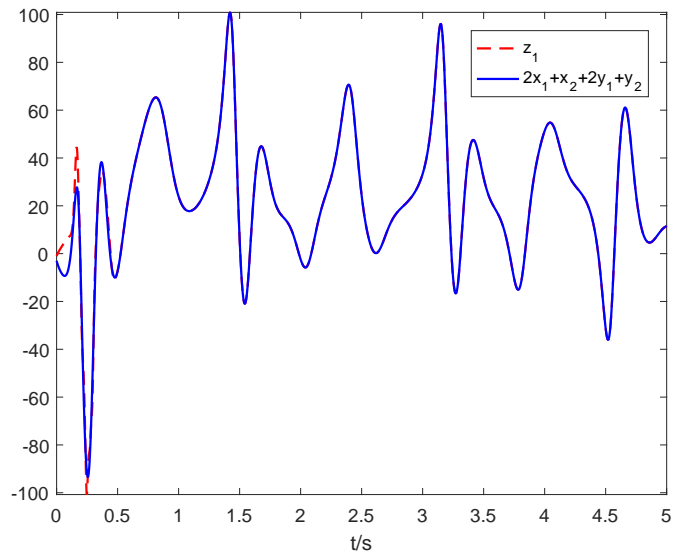

(a)

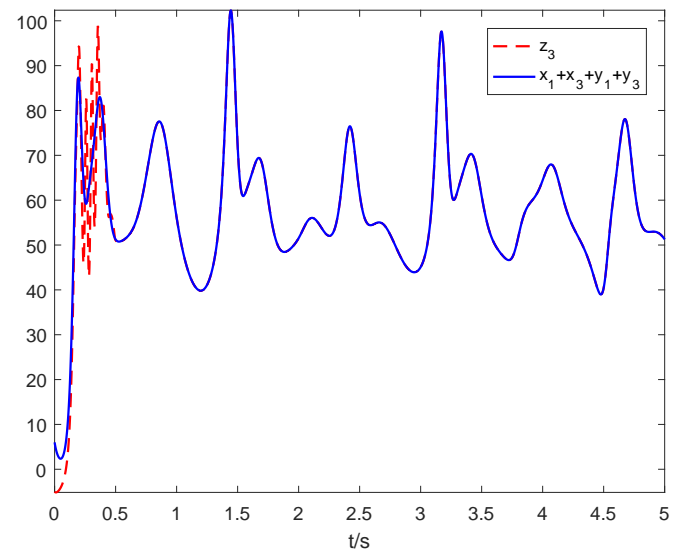

(c)

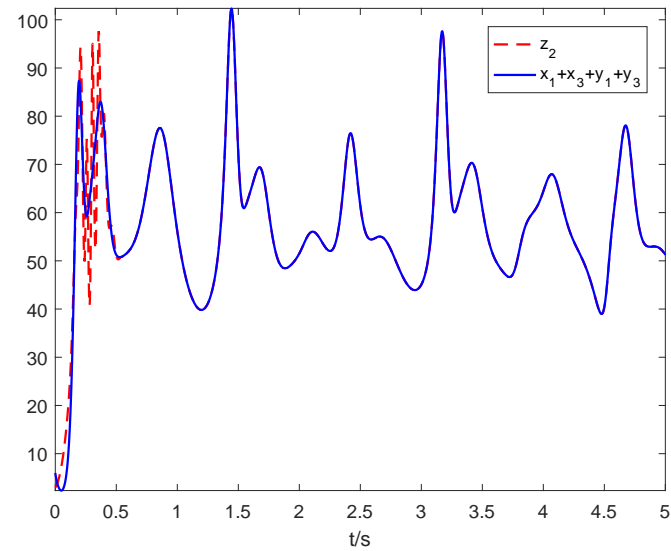

(b)

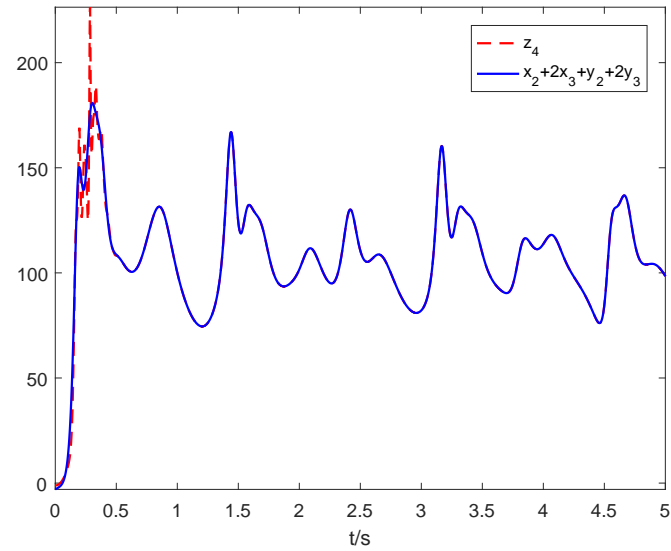

(d)

Fig.16. The synchronization for state variable $z_{1}$ and $2 x_{1}+x_{2}+2 y_{1}+y_{2}, z_{2}$ and $x_{1}+x_{3}+$ $y_{1}+y_{3}, z_{3}$ and $x_{1}+x_{3}+y_{1}+y_{3}, z_{4}$ and $x_{2}+2 x_{3}+y_{2}+2 y_{3}$ of drive systems (40), (41) and response system (42) indicating in sub-pictures (a)-(d) respectively.

$$
\begin{aligned}
\hat{a}_{1} & =-\left(x_{2}-x_{1}\right)\left\{{ }^{(311)} s_{31}+{ }^{(244)} s_{30}+2\left({ }^{(433)} s_{36}\right)\right\}-k_{2} \operatorname{sign}\left(\tilde{a}_{1}\right)\left(\operatorname{abs}\left(\tilde{a}_{1}\right)^{\omega}\right), \\
\hat{b}_{1} & =-x_{1}\left\{{ }^{(122)} s_{25}+{ }^{(433)} s_{36}\right\}-k_{2} \operatorname{sign}\left(\tilde{b}_{1}\right)\left(\operatorname{abs}\left(\tilde{b}_{1}\right)^{\omega}\right), \\
\hat{c}_{1} & =x_{3}\left\{{ }^{(244)} s_{30}+{ }^{(311)} s_{31}+2\left({ }^{(122)} s_{25}\right)\right\}-k_{1} \operatorname{sign}\left(\tilde{c}_{1}\right)\left(\operatorname{abs}\left(\tilde{c}_{1}\right)^{\omega}\right) \\
\hat{a}_{2} & =-\left(\left(y_{2}-y_{1}\right)\left\{{ }^{(244)} s_{30}+2\left({ }^{(433)} s_{36}\right)+{ }^{(311)} s_{31}\right\}-y_{1}\left\{{ }^{(122)} s_{25}+{ }^{(433)} s_{36}\right\}\right) \\
& -k_{3} \operatorname{sign}\left(\tilde{a}_{2}\right)\left(\operatorname{abs}\left(\tilde{a}_{2}\right)^{\omega}\right) \\
\hat{b}_{2} & =y_{3}\left\{{ }^{(244)} s_{30}+2\left({ }^{(122)} s_{25}\right)+{ }^{(311)} s_{31}\right\}-k_{3} \operatorname{sign}\left(\tilde{b}_{2}\right)\left(\operatorname{abs}\left(\tilde{b}_{2}\right)^{\omega}\right), \\
\hat{c}_{2} & =-\left(y_{1}+y_{2}\right)\left({ }^{(122)} s_{25}+{ }^{(433)} s_{36}\right)-k_{3} \operatorname{sign}\left(\tilde{c}_{2}\right)\left(\operatorname{abs}\left(\tilde{c}_{2}\right)^{\omega}\right), \\
\hat{a}_{3} & =\left(z_{2}-z_{1}\right)\left({ }^{(122)} s_{25}\right)-k_{4} \operatorname{sign}\left(\tilde{a}_{3}\right)\left(\operatorname{abs}\left(\tilde{a}_{3}\right)^{\omega}\right), \\
\hat{b}_{3} & =z_{2}\left({ }^{(244)} s_{30}\right)-k_{4} \operatorname{sign}\left(\tilde{b}_{3}\right)\left(\operatorname{abs}\left(\tilde{b}_{3}\right)^{\omega}\right), \\
\hat{c}_{3} & =-z_{3}\left({ }^{\left({ }^{(311)}\right.} s_{31}\right)-k_{4} \operatorname{sign}\left(\tilde{c}_{3}\right)\left(\operatorname{abs}\left(\tilde{c}_{3}\right)^{\omega}\right),
\end{aligned}
$$




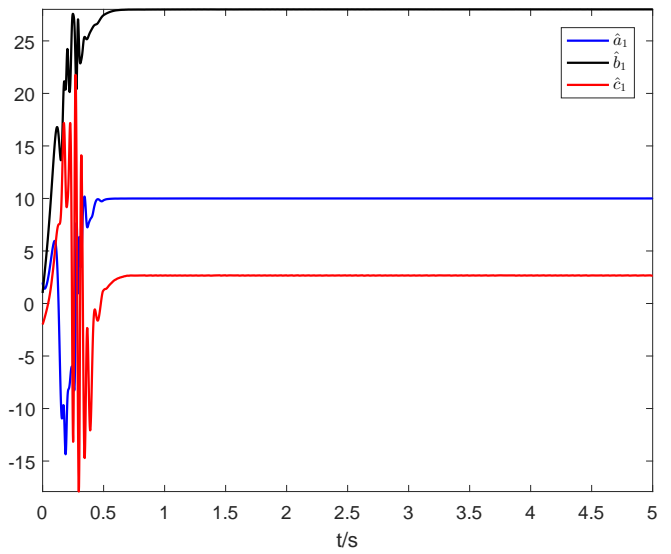

(a)

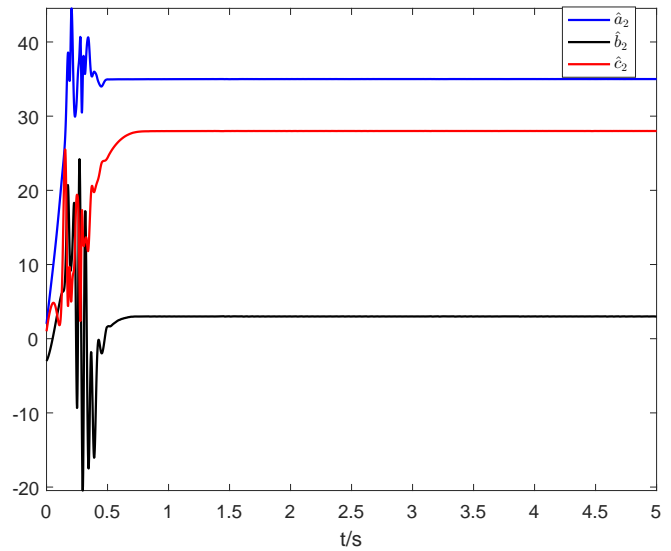

(b)

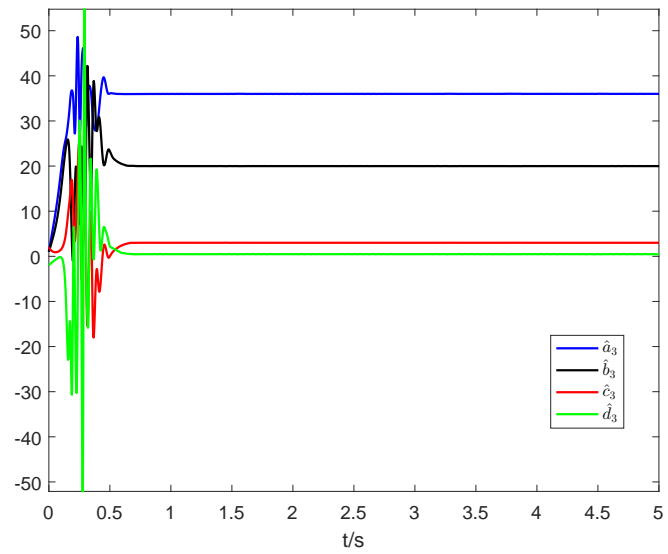

(c)

Fig.17. The estimation of parameters $\hat{a}_{1}, \hat{b}_{1}, \hat{c}_{1}$ of drive system (40) (a), $\hat{a}_{2}, \hat{b}_{2}, \hat{c}_{2}$ of drive system (41) (b), $\hat{a}_{3}, \hat{b}_{3}, \hat{c}_{3}, \hat{d}_{3}$ of drive system (43) (c).

$$
\hat{d}_{3}=z_{4}\left({ }^{(433)} s_{36}\right)-k_{4} \operatorname{sign}\left(\tilde{d}_{3}\right)\left(\operatorname{abs}\left(\tilde{d}_{3}\right)^{\omega}\right)
$$

In this two numerical simulations, the definition of matrices $A, B, C$ are shown in (43). The initial conditions of the drive systems (40), (41) and response system (42) are selected as $x(0)=(2,-2,1,1), y(0)=(1,1,2,2), z(0)=(-20,3,1,3)$. The initial values of the unknown parameter in $\mathrm{D}-\mathrm{R}$ system and upper bound of SD are selected as $\left(a_{1}(0), b_{1}(0), c_{1}(0), d_{1}(0)\right)=(2,1,-2,1),\left(a_{2}(0), b_{2}(0), c_{2}(0), d_{2}(0), r(0)\right)=$ $(1,-3,1,1,-5),\left(a_{3}(0), b_{3}(0), c_{3}(0), d_{3}(0)\right)=(1,1,-2,-2),\left(r_{1}(0), r_{2}(0), r_{3}(0), r_{4}(0)\right)=$ $(-2.5,1,-3,1),\left(q_{1}(0), q_{2}(0), q_{3}(0), q_{4}(0)\right)=(1,-2.5,-5,1)$. The constants ${ }^{(l w v)} \lambda_{p}=$ $2, m_{1}=10, m_{2}=10, k_{1}=10, k_{2}=10, k_{3}=10, k_{4}=10, \omega=0.5$. The trajectories about the error variables $\boldsymbol{e}=\left({ }^{(142)} e_{4},{ }^{(234)} e_{12},{ }^{(321)} e_{13},{ }^{\left({ }^{(413)}\right.} e_{21}\right)^{T}$ and $\boldsymbol{e}=$ $\left.{ }^{\left({ }^{(122)}\right.} e_{25},{ }^{(244)} e_{30},{ }^{(311)} e_{31},{ }^{(433)} e_{36}\right)^{T}$ are drawn in figures 11 and 15 . The synchronization for the state trajectories drive systems (40), (41) and response system (42) are drawn in figures 12 and 16. The trajectories of estimations $\hat{\boldsymbol{\theta}}, \hat{\boldsymbol{\beta}}, \hat{\boldsymbol{\vartheta}}$ are drawn in figures 13 and $17,{ }^{(l w v)} \rho_{p}$, and ${ }^{(l w v)} \varrho_{p}$ are drawn in figures 14 and 18 . Motivated by the numerical simulation results, it can reveal that the drive systems (4), (5) and response system (6) 


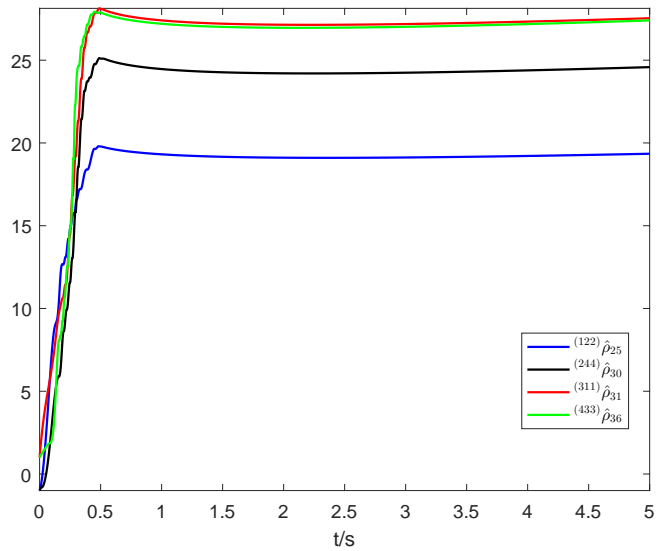

(a)

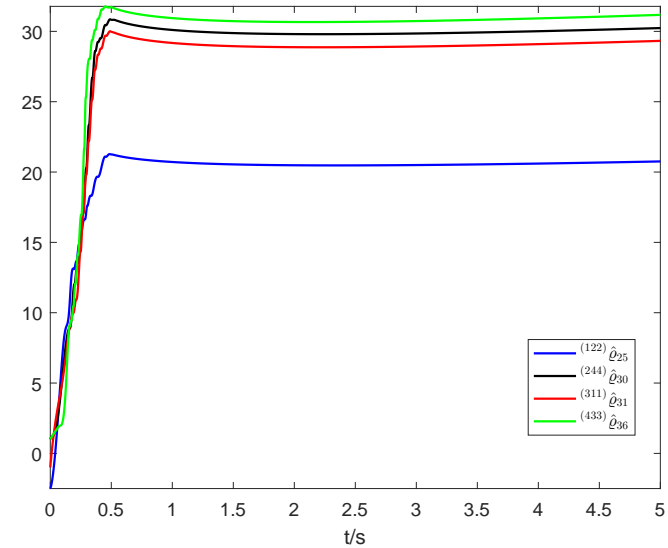

(b)

Fig. 18. The estimation of parameters ${ }^{(122)} \hat{\rho}_{25},{ }^{(244)} \hat{\rho}_{30},{ }^{(311)} \hat{\rho}_{31},{ }^{(433)} \hat{\rho}_{36}$ and ${ }^{(122)} \hat{\varrho}_{25},{ }^{(244)} \hat{\varrho}_{30}$, ${ }^{(311)} \hat{\varrho}_{31},{ }^{(433)} \hat{\varrho}_{36}$ shown in sub-pictures (a) and (b).

achieve MSSMCS. Therefore, the multi-switching adaptive controllers (MSAC) (25) and some suitable multi-switching adaptive updating laws (MSAUL) (28) are effective.

\section{Conclusion}

In this article, we investigated the multi-switching sliding mode combination synchronization (MSSMCS) of fractional order (FO) non-identical chaotic systems with unknown parameters under double stochastic disturbances (SD). In the theoretical parts, the FO chaotic systems with the different (or same )dimension are considered. Our idea for this topic is that with the help of the Lyapunov theory, adaptive control technology and sliding mode control technique, we put forward the fractional order sliding surface, multi-switching adaptive controllers (MSAC) and multi-switching adaptive updating laws (MSAUL) which can achieve the the state variables of the drive systems are synchronized with the different state variables of the response systems. Meanwhile, the unknown parameters are identified and upper bound of stochastic disturbances are examined accurately. What's more, the combination drive systems and single response system we chose are very general. The different description of the scale matrices can make the multi-switching projection synchronization, multi-switching complete synchronization, multi-switching anti-synchronization etc, become the special cases of MSSMCS. Motivated by the numerical simulation results, it is clear that the different error variables quickly converges to the equilibrium point. Therefore, the multi-switching adaptive controllers (MSAC) are effective and have strong robustness. Next, we will concentrate on the fractional order multi-switching synchronization of time-delay systems under multiple stochastic disturbances, and the parameters of system are still unknown.

\section{Declarations}


Funding: This work is partly supported by Sichuan Youth Science and Technology Foundation (Grant No. 2019YJ0456), Fund of Sichuan University of Science and Engineering (Grant No. 2020RC26, 2020RC42).

Competing interests: The authors declare that they have no competing interests.

Availability of data and material: The data used to support the findings of this study are available from the corresponding author upon request.

\section{References}

[1] Ping, Z., Peng, Z.: Drive-response synchronization for chaotic systems. J. Chong qing Unive. 25, 77-79 (2002)

[2] Yu, J., Hu, C., Jiang, H.: Projective synchronization for fractional neural networks. Neural Netw. 49, 87-95 (2014)

[3] Shao, K., Guo, H., Han, F.: Finite-time projective synchronization of fractionalorder chaotic systems via soft variable structure control. J. Mech. Sci. Technol. 34, 369-376 (2020)

[4] Qin, X., Li, S., Liu, H.: Adaptive fuzzy synchronization of uncertain fractionalorder chaotic systems with different structures and time-delays. Adv. Diff. Equ. 2019(1), 1-16 (2019)

[5] Bouzeriba, A., Boulkroune, A., Bouden, T.: Fuzzy adaptive synchronization of a class of fractional-order chaotic systems. Int. J. Mach. Learn. Cybern. 7, 1-16 (2016)

[6] Liu, Y.J., Gong, M., Tong, S., et al.: Adaptive fuzzy output feedback control for a class of nonlinear systems with full state constraints. IEEE Trans. Fuzzy. Syst. 26, 2607-2617 (2018)

[7] Ha, S., Chen, L., Liu, H.: Command filtered adaptive neural network synchronization control of fractional-order chaotic systems subject to unknown dead zones. J. Franklin Inst. 358(7), 3376-3402 (2021)

[8] Zeng, H.B., Teo, K.L., He, Y., et al.: Sampled-data synchronization control for chaotic neural networks subject to actuator saturation. Neurocomputing, 185, 1656-1667 (2017)

[9] Wang, J., Xu, C.: Stochastic feedback coupling synchronization of networked harmonic oscillators. Automatica, 87, 404-411 (2018)

[10] Li, H.L., Jiang, Y.L., Wang, Z., et al.: Parameter identification and adaptiveimpulsive synchronization of uncertain complex networks with nonidentical topological structures. Optik-Int. J. Light Electron Opt. 126, 5771-5776 (2015)

[11] Zhang, H.L., et al.: Synchronization of uncertain chaotic systems via completeadaptive-impulsive controls. Chaos. Solitons Fractals. Appl. Sci \& Eng. Interdiscip. J. Nonlinear Sci. 100, 24-30 (2017) 
[12] Kocamaz, U.E., Cevher, B., et al.: Control and synchronization of chaos with sliding mode control based on cubic reaching rule. Chaos. Solitons Fractals. 105, 92-98 (2017)

[13] Vaidyanathan, S.: Anti-synchronization of 3-cells cellular neural network attractors via integral sliding mode control. Int. J. PharmTech Res. 9, 193-205 (2016)

[14] Li, X., Zhao, X.S.: The chaotic synchronization of fractional-order and integerorder in a class of financial systems. J. Sci. Teach. Coll. Univ. 40, 1-4 (2020)

[15] Jing, W., Guang, P.: Design of a sliding mode controller for synchronization of fractional-order chaotic systems with different structures. J. Shanghai Jiaotong Univ. 50, 849-853+860 (2016)

[16] Jiang, N.: The adaptive control synchronization of hyper-chaos lorenz system and hyper-chaos Rössler system. J. Taiyuan Norm Univ. 013, 47-50 (2014)

[17] Wei, X.: Adaptive control and synchronization of Lü hyper-chaotic system. J. Honghe Univ. 13, 23-27 (2015)

[18] Li, T., Wang, Y., Zhao, C.: Synchronization of fractional chaotic systems based on a simple Lyapunov function. Adv. Diff. Equ. 2017, 304 (2017)

[19] Wei, Y.H., Chen, Y.Q.: Lyapunov functions for nabla discrete fractional order systems. ISA Trans. 88, 82-90 (2019)

[20] Khan, A., Jahanzaib, L.S.: Synchronization on the adaptive sliding mode controller for fractional order complex chaotic systems with uncertainty and disturbances. Int. J. Dyn. Control. 7, 1419-1433 (2019)

[21] Luo, R., Su, H., Zeng, Y.: Synchronization of uncertain fractional-order chaotic systems via a novel adaptive controller. Chin. J. Phys. 55, 342-349 (2017)

[22] Zhang, R., Yang, S.: Robust chaos synchronization of fractional-order chaotic systems with unknown parameters and uncertain perturbations. Nonlinear Dyn. 69, 983-992 (2012)

[23] Ma, S.J., Shen, Q., Jing, H.: Modified projective synchronization of stochastic fractional order chaotic systems with uncertain parameters. Nonlinear Dyn. 73, 93-100 (2013)

[24] Wang, Q., Qi, D.L.: Synchronization for fractional order chaotic systems with uncertain parameters. Int. J. Control. Autom. Syst. 14, 211-216 (2016)

[25] Nian, F., Liu, X., Zhang, Y.: Sliding mode synchronization of fractional-order complex chaotic system with parametric and external disturbances. Chaos. Solitons Fractals. 116, 22-28 (2018)

[26] Zhang, X., Zhang, X., Li, D., et al.: Adaptive Synchronization for a Class of Fractional Order Time-delay Uncertain Chaotic Systems via Fuzzy Fractional Order Neural Network. Int. J. Control. Autom. Syst. 17, 1209-1220 (2019) 
[27] Deepika, D., Sandeep, K., Shiv, N.: Uncertainty and disturbance estimator based robust synchronization for a class of uncertain fractional chaotic system via fractional order sliding mode control. Chaos. Solitons Fractals. 115, 196-203 (2018)

[28] Luo, R., Wang, Y.L., Deng, S.C.: Combination synchronization of three classic chaotic systems using active backstepping design. Chaos (Woodbury, N.Y.). 21, 043114 (2011)

[29] Khan, A., Nigar, U.: Adaptive hybrid complex projective combinationcombination synchronization in non-identical hyper-chaotic complex systems. Int. J. Dynam. Control. 7, 1404-1418 (2019)

[30] Vincent, U.E., Saseyi, A.O., Mcclintock, P.: Multi-switching combination synchronization of chaotic systems. Nonlinear Dyn. 80, 845-854 (2015)

[31] Sun, J., Cui, G., Wang, Y., et al.: Combination complex synchronization of three chaotic complex systems. Nonlinear Dyn. 79, 953-965 (2015)

[32] Khan, A., Budhraja, M., Ibraheem, A.: Combination-combination synchronisation of time-delay chaotic systems for unknown parameters with uncertainties and external disturbances. Pramana-J Phys. 91, 1-11 (2018)

[33] Sun, J., Yan, W., Wang, Y., et al.: Compound-combination synchronization of five chaotic systems via nonlinear control. Optik-Int. J. Light and Electron Opt. 127, 4136-4143 (2016)

[34] Khan, A., Trikha, P.: Compound difference anti-synchronization between chaotic systems of integer and fractional order. SN Appl. Sci. 1, 1-13 (2019)

[35] Yadav, V.K., Prasad, G., Srivastava, M., et al.: Triple Compound Synchronization Among Eight Chaotic Systems with External Disturbances via Nonlinear Approach. Differ. Equations. Dyn. Syst. 2019, 1-24 (2019)

[36] Zhang, B., Deng, F.: Double-compound synchronization of six memristor-based Lorenz systems. Nonlinear Dyn. 77, 1519-1530 (2014)

[37] Mahmoud, G.M., Abed-Elhameed, T.M., Farghaly, A.A.: Double compound combination synchronization among eight n-dimensional chaotic systems. Chin. Phys. B. 27, 154-162 (2018)

[38] Vincent, U.E., Saseyi, A.O., Mcclintock, P.: Multi-switching combination synchronization of chaotic systems. Nonlinear Dyn. 80 845-854 (2015)

[39] Khan, A., Khattar, D., Prajapati, N.: Dual combination combination multi switching synchronization of eight chaotic systems. Chin. J. Phys. 55, 1209-1218 (2017)

[40] Ahmad, I., Shafiq, M., Al-Sawalha, M.M.: Globally exponential multi switchingcombination synchronization control of chaotic systems for secure communications. Chin. J. Phys. 56, 974-987 (2018) 
[41] Zheng, S.: Multi-switching combination synchronization of three different chaotic systems via nonlinear control. Optik-Int. J. Light and Electron Opt. 127, 1024710258 (2016)

[42] Khan, A., Budhraja, M., Ibraheem, A.: Synchronization Among Different Switches of Four Non-identical Chaotic Systems via Adaptive Control. Arabian J. Sci. Eng. 44, 2717-2728 (2019)

[43] Aysha, I.: Multi-switching Dual Combination Synchronization of Time Delay Dynamical Systems for Fully Unknown Parameters via Adaptive Control. Arabian J. Sci. Eng. 45, 6911-6922 (2020)

[44] Shafiq, M., Ahmad, I.: Multi-Switching Combination Anti-synchronization of Unknown Hyper-chaotic Systems. Arabian J. Sci. Eng. 44, 1-16 (2019)

[45] Song, S., Song, X.N., Pathak, N., et al.: Multi-switching adaptive synchronization of two fractional-order chaotic systems with different structure and different order. Int. J. Control. Autom. Syst. 15, 1524-1535 (2017)

[46] Shahzad, M.: Multi-switching Synchronization of Different Orders: A Generalization of Increased/Reduced Order Synchronization. Iran. J. Sci. technol. 44, 167-176 (2020)

[47] Chen, X.Y., Ju, H., et al.: Adaptive synchronization of multiple uncertain coupled chaotic systems via sliding mode control. Neurocomputing. 273, 9-21 (2018)

[48] Chen, X., Cao, J., Park, J.H., et al.: Finite-time multi-switching synchronization behavior for multiple chaotic systems with network transmission mode. J. Franklin Inst. 355, 2892-2911 (2018)

[49] Podlubny, I.: Fractional Differential Equations. New York. Academic. (1999)

[50] Li, Y., Chen, Y.Q., Podlubny, I.: Stability of fractional-order nonlinear dynamic systems: Lyapunov direct method and generalized Mittag-Leffler stability. Pergamon Press. Inc 59, 1810-1821 (2009) 